\title{
On the holonomy of Lorentzian metrics
}

\author{
Charles Boubel ${ }^{1}$
}

\begin{abstract}
Indecomposable Lorentzian holonomy algebras, except $\mathfrak{s o}(n, 1)$ and $\{0\}$, are not semisimple; they possibly belong to four families of algebras. All four families are realized as families of holonomy algebras: we describe the corresponding set of germs of metrics in each case.

Résumé. Les algèbres d'holonomie lorentziennes indécomposables, exceptées $\mathfrak{s o}(n, 1)$ et $\{0\}$, ne sont pas semi-simples. Elles se classent en quatre familles possibles. Ces quatre familles sont effectivement réalisées commes familles d'algèbres d'holonomie : nous décrivons, pour chacune d'elles, l'ensemble correspondant de germes de métriques.
\end{abstract}

\section{Introduction}

In [BBI93], L. Bérard Bergery and A. Ikemakhen showed that four families of linear representations may be realized as the holonomy representation of an indecomposable Lorentzian manifold. All four types are realized as such holonomy representations: we express each of them as a differential condition on the germ of metric. This is Theorem 5.14, proven thanks to some "adapted" coordinates built by Theorem 3.7. It follows a parametrization of the set of germs of metrics in each case. In this introduction we recall the context of the question and its motivation; $\S 2$ recalls precisely the result of [BBI93] and adjacent remarks. Then $\S 3$ presents the adapted coordinates and $\S 4$ builts them; $\S 5$ states the main theorem, proven then in $\S 6$. In $\S 7$, Corollary 7.2 reformulates Theorem 3.7 in terms of a parametrization of a set of germs of metrics; together are given additional comments and explicit examples.

\section{1. a The holonomy group: definition, problematics}

Let $\mathcal{M}$ be a differential manifold. With any affine connection $D$ on $\mathcal{M}$ is associated, after Elie Cartan, see [C24] and [C26], its holonomy group $H$, as follows. The connection defines a parallel transport of each vector $V \in \mathrm{T}_{p} \mathcal{M}$ along any (regular) curve $\gamma$ in $(\mathcal{M}, D)$, based at $p$ : it is the unique vector field $\widetilde{V}$ along $\gamma$ such that $\widetilde{V}(p)=V$ and $D_{\gamma^{\prime}} \widetilde{V}=0$. Denoting by $q$ the other end of $\gamma$, this parallel transport defines a linear isomorphism $\tau_{\gamma}: \mathrm{T}_{p} \mathcal{M} \mapsto \mathrm{T}_{q} \mathcal{M}$. The restricted holonomy group $H_{p}^{0}$ of $(\mathcal{M}, D)$ at the point $p$ is defined as the group of the $\tau_{\gamma}$, for all the loops $\gamma$ based at $p$ and homotopic to a constant. It is a Lie group immersed in $\mathrm{GL}\left(\mathrm{T}_{p} \mathcal{M}\right)$. For any $p^{\prime}$ of $\mathcal{M}, H_{p}^{0}$ and $H_{p^{\prime}}^{0}$ are conjugated by the parallel transport along any curve from $p$ to $p^{\prime}$, so the holonomy group $H^{0}$ of $(\mathcal{M}, D)$ is defined, independently of the base point, as a conjugacy class of linear representations in $\mathbb{R}^{n}$, where $n=\operatorname{dim} \mathcal{M}$. We do not focus here on the full holonomy group $H_{p}$ generated by the $\tau_{\gamma}$ for all loops $\gamma$ based at $p$; it is also a Lie group, $H_{p}^{0}$ is its neutral connected component. The main question linked with it is to know which representations of a Lie group in $\mathbb{R}^{n}$ are realized as a holonomy representation, and to which geometrical properties of $D$ they correspond.

Actually, every representation can arise as a holonomy representation, see [HO56]. Assuming, as in the following, that $D$ is torsion free, makes the question non-trivial.

\section{1.b The Riemannian, and more generally irreducible case, is solved}

If $D$ is the Levi-Civita connection of a (pseudo-)Riemannian metric, by [dR52] and its pseudo-Riemannian generalization [W67], the universal cover of a decomposable (geodesi-

\footnotetext{
${ }^{1}$ Unité de Mathématiques Pures et Appliquées (UMR CNRS 5669), École Normale Supérieure de Lyon, 46 allée d'Italie 69364 LYON CEDEX 07 FRANCE Charles.Boubel@ens-lyon.fr
} 
cally complete) manifold is a Riemannian product, each factor being the exponential of one term of the holonomy decomposition of $\mathrm{T}_{p} \mathcal{M}$. We recall some terminology.

1.1 Terminology If $g$ is a (pseudo-)euclidian product on $\mathbb{R}^{n}$, a $g$-orthogonal group representation in $\mathbb{R}^{n}$ is called (in)decomposable if it is (not) a direct $g$-orthogonal sum of two subrepresentations. By an immediate induction, any $g$-orthogonal representation is a direct orthogonal sum of indecomposable ones. As usual, a representation stabilizing no proper subspace is called irreducible. A (pseudo-)Riemannian manifold is called (locally) indecomposable, respectively irreducible, if its (restricted) holonomy representation is.

So it is sufficient to study the holonomy of the indecomposable (pseudo-)Riemannian manifolds; note also that an indecomposable Riemannian manifold is necessarily irreducible.

The irreducible case is now completely understood. Good surveys of the topic are available, see e.g. [Bes87] ch.10, [S01a] and above all [S01b] and [Br96]. On the opposite, the general case, i.e. indecomposable but possibly reducible, remains nearly unexplored; it does not stem from fundamental reasons, but from technical ones, see [S01a] p. $61 \S 2$.

\section{1. c The Lorentzian case, topic of this work}

Now, what are the holonomy representations of Lorentzian indecomposable manifolds, i.e. pseudo-Riemannian with signature $(n-1,1)$ ? The classification in the irreducible case brings no answer. Indeed, indecomposable pseudo-Riemannian manifolds may be reducible: certainly if $E$ is a $H_{p}^{0}$-stable proper subspace of $\mathrm{T}_{p} \mathcal{M}$, so is $E^{\perp}$, but if $g_{\mid E}$ is degenerate, $E^{\perp}$ is not a complement of $E$ in $\mathrm{T}_{p} \mathcal{M}$. In the Lorentzian case, this "reducible-indecomposable" situation is even the only non-trivial one: it follows from Berger's classification [Be55, Be57], together with Cahen and Parker's work [CP80] that:

1.2 Proposition Let $(\mathcal{M}, g)$ be an indecomposable Lorentzian manifold and $H^{0}$ its restricted holonomy group. Then

- either $H^{0}=S O_{n-1,1}^{0}(\mathbb{R})$ ("generic case")

- or the representation of $H^{0}$ in $\mathrm{T}_{p} \mathcal{M}$ is reducible.

See also direct proofs of this in [DO01], [Z02] or [BZ03]. So in that sense, the behaviour of the Lorentzian holonomy groups is the opposite of that of the Riemannian ones.

With this in mind, L. Bérard Bergery and A. Ikemakhen classified in four families the indecomposable representations of a Lie group in $\mathbb{R}^{n}$, preserving a Lorentzian product, see [BBI93] and $\S 2$. b here. If they are realized as holonomy representations, to which families of metrics do those families of representations correspond? This is the topic of this paper.

Theorem 5.14 p. 21 answers the question. In coordinates "adapted" to the situation built, and this is a quite technical preliminary, by Theorem 3.7 p. 7 -, it gives a necessary and sufficient condition for the metric to be in each of the four families.

This condition involves, in the case of the "exceptional" families with parameters (types 3 and 4 after the terminology given by Theorem 2.1 p. 4), a very peculiar constraint on some families of Kähler metrics appearing on a quotient of the manifold (then called "admissible families, see Definition 5.4 p. 19), as well as a differential link (see Definition 5.9 p. 20) between those families of Kähler metrics and a 1-form $\gamma$ appearing in the metric written in adapted coordinates, see formula (3.1) p. 6. It has to be noticed that this form $\gamma$ has an intrinsic significance given by Remark 7.5 p. 30 and Proposition 7.4. 
The condition given by Theorem 5.14 for a metric to be in one of the "exceptional" families 3 or 4 enables then to give a parametrization of the set of germs of metrics with holonomy in these families, up to an action of a group of the type $\prod_{i} \mathrm{SO}_{n_{i}}(\mathbb{R}) \times \mathbb{R}^{n_{i}}$. This is Corollary 5.15 p. 21. The parameters are some 1-parameter families of metrics on some quotients of the manifold $\mathcal{M}$ and a 1 -form given on a submanifold of $\mathcal{M}$.

We give also, similarly, a parametrization of the whole set of the reducible-indecomposable Lorentzian metrics, giving additionally an intrinsic sense to one of the parameters, see Corollary 7.2 p. 28 and Proposition 7.4 p. 30.

Finally, in a particular case, the differential relation introduced in Definition 5.9 p. 20, and characterizing the "exceptional" families of metrics, takes a more explicit meaning, see section 7.b p. 30. Using Theorem 3.7, we also give, in low dimension, explicit examples of metrics with holonomy of type 3 and 4 , see section 7 .c p. 31 .

1.3 Remarks (i) General "indecomposable-reducible" pseudo-Riemannian manifolds are very complicated: $H^{0}$ is not semi-simple, an arbitrary number of subspaces may be stabilized, with interlinked inclusion relations... No simple classification should be hoped.

(ii) Unlike the irreducible case, solved using high-level classical machineries - Representation Theory, Exterior Differential Systems and others-, the present reducible-indecomposable case turned out to be solved by (a lot of) elementary Differential Calculus. Another, unfortunate, difference is that the obtained results cannot be stated in a simple way: the involved germs of metrics seem to be intrinsically complicated to describe.

(iii) The elementary calculus used here has yet an advantage: it describes sets of germs of metrics corresponding to a given holonomy type, in the $C^{\infty}$ class instead of the analytic class, usual framework for such matters.

Thanks. A significant part of this work was done during a Ph.D. [Bo00] supervised by L. Bérard Bergery whom I thank for having introduced me to that problematics. I thank B. Sévennec, J.-C. Sikorav and É. Ghys for their help, technical and about the writing. Be also thanked all relatives and friends who gave a precious human, extra-mathematical support.

\section{Precise setup of the problem}

A quite detailed local description of Lorentzian reducible-indecomposable metrics follows from basic standard remarks. It may be not familiar to all reader, and our whole notation is based on it, so we recall it, together with the algebraic result of [BBI93].

\section{2. a Basic facts about Lorentzian reducible-indecomposable metrics}

Foliations associated with holonomy-stable subspaces. Let $(\mathcal{M}, g)$ be a pseudo-Riemannian manifold and $m \in \mathcal{M}$. It follows from the definition of the holonomy group in $\S 1$. a that a subspace $\mathbf{E}$ of $\mathrm{T}_{m} \mathcal{M}$ is holonomy-stable if and only if it induces, by parallel transport, a parallel distribution $\mathbf{E}$ on $(\mathcal{M}, g)$ - on any simply connected domain of $\mathcal{M}$ in the case of the restricted holonomy group. As the Levi-Civita connection $D$ is torsion free, $\mathbf{E}$ is integrable; as $\mathbf{E}$ is parallel, its integral leaves are totally geodesic.

If $(\mathcal{M}, g)$ is indecomposable, by definition, $\mathbf{E}$ is necessarily degenerate i.e. $g_{\mid E}$ is. Consequently, the following spaces are also $H$-stable (possibly among many others) :

$$
\{0\} \subset \mathbf{E} \cap \mathbf{E}^{\perp}\left\{\begin{array}{l}
\subset \mathbf{E} \\
\subset \mathbf{E}^{\perp}
\end{array}\right\} \subset \mathbf{E}+\mathbf{E}^{\perp} \subset \mathrm{T}_{m} \mathcal{M} .
$$


The associated foliations with totally geodesic, degenerate leaves play a crucial role in the geometry of the "reducible-indecomposable" pseudo-Riemannian manifolds.

The Lorentzian case - Notation. Now let $g$ be indecomposable and Lorentzian, i.e. $\operatorname{dim} \mathcal{M}=n, \operatorname{sign}(g)=(n-1,1)$. Take $\mathbf{E}$ as above; $\operatorname{ker}\left(g_{\mid \mathbf{E}}\right)=\mathbf{E} \cap \mathbf{E}^{\perp}$ is totally isotropic, so necessarily one-dimensional. If another isotropic line $\mathbf{F}$ were also stable, the sum $\operatorname{ker}\left(g_{\mid \mathbf{E}}\right) \oplus \mathbf{F}$ would be stable and nondegenerate; this is excluded by assumption, except if $n=2$ where the problem of holonomy is trivial. So, a reducible-indecomposable Lorentzian manifold $(n>2)$ admits a unique isotropic vectorial line stable by holonomy, now denoted by $\mathbf{X}_{m}$. Its orthogonal $\mathbf{X}_{m}^{\perp}$ is a degenerate hyperplane of $\mathrm{T}_{m} \mathcal{M}$ with signature $(n-2,0)$; so the following flag in $\mathrm{T}_{m} \mathcal{M}$ is canonical and holonomy-stable:

$$
\{0\} \subset \mathbf{X}_{m} \subset \mathbf{X}_{m}^{\perp} \subset \mathrm{T}_{m} \mathcal{M}
$$

\section{2. b Algebraic situation - Notation}

As $H^{0}$ is connected, we focus on its holonomy algebra $\mathfrak{h}$. Let $\beta=\left(X,\left(Y_{i}\right)_{i=1}^{n-2}, Z\right)$ be a basis of $\mathrm{T}_{m} \mathcal{M}$ such that $\operatorname{span}(X)=\mathbf{X}_{m}, \operatorname{span}\left(X,\left(Y_{i}\right)_{i=1}^{n-2}\right)=\mathbf{X}_{m}^{\perp}$ and moreover that $g(Z, X)=1$ and that $Z \perp \operatorname{span}\left(\left(Y_{i}\right)_{i=1}^{n-2}, Z\right)$; in particular, $Z$ is isotropic. The holonomy algebra $\mathfrak{h}$ is included in the subalgebra $\mathfrak{g}$ of $\mathfrak{s o}(g)$ stabilizing $\mathbf{X}_{m}$. In such a basis $\beta$ :

$$
\operatorname{Mat}_{\beta}(g)=\left(\begin{array}{ccc}
0 & 0 & 1 \\
0 & I_{n-2} & 0 \\
1 & 0 & 0
\end{array}\right) \quad \text { and } \quad h \in \mathfrak{g} \Leftrightarrow \operatorname{Mat}_{\beta}(h)=\left(\begin{array}{ccc}
a & L & 0 \\
0 & A & -{ }^{t} L \\
0 & 0 & -a
\end{array}\right)
$$

with $(a, L, A) \in \mathbb{R} \times \mathbb{R}^{n-2} \times \mathfrak{s o}_{n-2}(\mathbb{R})$. Here is the algebraic result this work is based on.

2.1 Theorem [BBI93] Let $\mathfrak{b}$ be a subalgebra of $\mathfrak{g}$. The action of $\mathfrak{b}$ on $\mathrm{T}_{m} \mathcal{M}$ is reducible and indecomposable if and only if, written in a (well-chosen, for type 4) basis $\beta$ of the type described above, $\mathfrak{b}$ is of one of the four following types:

$$
\begin{aligned}
& 1\left\{\left(\begin{array}{ccc}
a & L & 0 \\
0 & C & -{ }^{t} L \\
0 & 0 & -a
\end{array}\right) \text { with }(a, L, C) \in \mathbb{R} \times \mathbb{R}^{n-2} \times \mathfrak{c}\right\} \text { with } \mathfrak{c} \text { a subalgebra of } \mathfrak{s o}_{n-2}(\mathbb{R}), \\
& 2\left\{\left(\begin{array}{ccc}
0 & L & 0 \\
0 & C & -{ }^{t} L \\
0 & 0 & -0
\end{array}\right) \text { with }(L, C) \in \mathbb{R}^{n-2} \times \mathfrak{c}\right\} \text { with } \mathfrak{c} \text { a subalgebra of } \mathfrak{s o}_{n-2}(\mathbb{R}), \\
& 3\left\{\left(\begin{array}{ccc}
\psi(R) & L & 0 \\
0 & R & -{ }^{t} L \\
0 & 0 & -\psi(R)
\end{array}\right) \text { with }(L, R) \in \mathbb{R}^{n-2} \times \mathfrak{r}\right\} \text { with } \mathfrak{r} \text { a reductive subalgebra of }
\end{aligned}
$$
$\mathfrak{s o}_{n-2}(\mathbb{R})$ and $\psi$ a non zero linear form on it,

$$
4\left\{\left(\begin{array}{cccc}
0 & \psi(R) & L & 0 \\
0 & 0 & 0 & -{ }^{t} \psi(R) \\
0 & 0 & R & -{ }^{t} L \\
0 & 0 & 0 & 0
\end{array}\right) \text { with }(L, R) \in \mathbb{R}^{d_{2}} \times \mathfrak{r}\right\} \text { with } \mathfrak{r} \text { a reductive subalgebra }
$$

of $\mathfrak{s o}_{d_{2}}(\mathbb{R}), d_{2} \geqslant 2$, and $\psi$ a linear map from $\mathfrak{r}$ onto $\mathbb{R}^{d_{1}}, d_{1} \geqslant 1 ; d_{1}+d_{2}=n-2$.

2.2 Terminology In case 4 , we call here the subspace spanned by the vectors correponding to the first two blocks of the matrices "the binded subspace" of $\mathrm{T}_{m} \mathcal{M}$. 
The problem is not empty: Lorentzian metrics with holonomy algebra of type 1 and 2 may be easily written in local coordinates or built as homogeneous spaces. They are in some sense generic among reducible-indecomposable Lorentzian metrics. The difficult point is to understand to which differential property of the metric do holonomy representations of types 3 or 4 correspond. Particular examples are given in [I96], 4.1.3 and 4.1.4. but it does not answer the question.

2.3 Note A related problem is to determine which subalgebras of $\mathfrak{s o}(n-1,1)$ are realized as holonomy representations, i.e. which algebras $\mathfrak{r}$, as introduced in Theorem 2.1, may appear. This problem is now solved. After T. Leistner [L03a, L03b], $\mathfrak{r}$ has to be a Riemannian holonomy algebra. Conversely, holonomy representations of type 1 or 2 and any arbitrary Riemannian holonomy algebra $\mathfrak{r}$ acting on $\mathbf{X}_{m}^{\perp} / \mathbf{X}_{m}$ may be easily realized by some metrics in local coordinates, see [BBI93] §5 p.37-38. On his side, A. Galaev provided recently metrics of type 3 and 4 with any Riemannian holonomy algebra $r$ acting on $\mathbf{X}_{m}^{\perp} / \mathbf{X}_{m}$, for which type 3 and 4 make sense, i.e. such that the dimention of the center of $\mathfrak{r}$ is greater or equal to one (for type 3) or to the dimension of the binded subspace (for type 4), see [G05]. Lemma 5.1 p. 18 gives here the form of those algebras.

2.4 Notation Throughout, we will denote

- vectors or vector fields by light-faced capitals: $X, Y, V \ldots$ If $\left(x, y_{i} \ldots\right)$ are coordinates, the corresponding uppercase letters denote the associated coordinate vectors: $X=\frac{\partial}{\partial x} \ldots$

- distributions of subspaces in the tangent bundle by bold-faced capitals: $\mathbf{X}, \mathbf{Y}$; their fibre over a point $p$ by $\mathbf{X}_{p} \subset T_{p} \mathcal{M}, \mathbf{Y}_{p} \subset T_{p} \mathcal{M}$; if they are integrable, their integral foliation by the corresponding cursive capital: $\mathcal{X}$, and by $\mathcal{X}_{p}$ its leaf through $p$.

At any point $p, \pi$ is the projection $T_{p} \mathcal{M} \rightarrow T_{p} \mathcal{M} / \mathbf{X}_{p}$. Points, subsets of, or tensors on, the quotient $\mathrm{T} \mathcal{M} / \mathbf{X}$ are distinguished by a check: $\pi(p)=\check{p}, \mathbf{X}_{p}^{\perp} / \mathbf{X}_{p}=\pi\left(\mathbf{X}_{p}^{\perp}\right)=\check{\mathbf{X}}^{\perp} \ldots$

Canonical sub-foliations - Further notation. The induced metric $\check{g}$ on $\check{\mathbf{X}}^{\perp}=\mathbf{X}^{\perp} / \mathbf{X}$ is nondegenerate; as $g$ is Lorentzian, $\check{g}$ is even positive definite. The representation of the restricted holonomy group $H_{m}^{0}$ on $\check{\mathbf{X}}_{m}^{\perp}$ is thus totally reducible; we denote by $\check{H}_{m}$ the induced subgroup of $\mathrm{SO}\left(\check{\mathbf{X}}_{m}^{\perp}, \check{g}_{m}\right)$. Moreover:

2.5 Proposition ([BBI93], p. 36) $\check{\mathbf{X}}_{m}^{\perp}$ and $\check{H}_{m}$ admit respective decompositions

$$
\left.\check{\mathbf{X}}_{m}^{\perp}=\underset{0 \leqslant s \leqslant k}{\oplus} \check{\mathbf{Y}}_{m}^{s} \quad \text { (with possibly } \operatorname{dim} \check{\mathbf{Y}}_{m}^{0}=0\right) \text { and } \quad \check{H}_{m}=\prod_{1 \leqslant s \leqslant k} \check{H}_{m}^{s}
$$

where, for each $s \in \llbracket 1, k \rrbracket, \check{H}_{m}^{s}$ acts irreducibly on $\check{\mathbf{Y}}_{m}^{s}$ and trivially on the $\check{\mathbf{Y}}_{m}^{r}$, for $r \neq s$. In particular, $\check{H}_{m}$ acts trivially on $\check{\mathbf{Y}}_{m}^{0}$.

This property, known as "Borel-Lichnérowicz property", is satisfied by the holonomy representation of the totally orthogonally reducible (pseudo-)Riemannian manifolds. It has no reason to hold for a quotient holonomy representation on $\mathbf{E}_{m} /\left(\mathbf{E}_{m} \cap \mathbf{E}_{m}^{\perp}\right)$, except if $\operatorname{dim}\left(\mathbf{E}_{m} \cap \mathbf{E}_{m}^{\perp}\right)=1$; then it follows immediately from the Ambrose-Singer theorem, see [BBI93] p. 36. Proposition 2.5 gives a first basic but essential fact:

2.6 Corollary Decomposition (2.2) of $\check{\mathbf{X}}^{\perp}$ is canonical: $\check{\mathbf{Y}}_{m}^{0}$ is the trivial factor of the action of $\check{H}_{m}^{0}$ and $\left(\check{\mathbf{Y}}_{m}^{s}\right)_{s=1}^{k}$ the unique strongest $H_{m}^{0}$-stable decomposition of $\left(\check{\mathbf{Y}}_{m}^{0}\right)^{\perp}$. 
We now set, for each $s, n_{s}=\operatorname{dim} \check{\mathbf{Y}}^{s}$ and $\mathbf{Y}^{s}=\pi^{-1}\left(\check{\mathbf{Y}}^{s}\right) \subset \mathrm{T}_{m} \mathcal{M}$. Similarly, the $\mathbf{Y}^{s}$ induce integral foliations, with degenerate, totally geodesic leaves, denoted by $\mathcal{Y}^{s}$. The projection $\mathcal{M} \rightarrow \mathcal{M} / \mathcal{X}$, defined locally around $m$, is denoted by $\pi$, like its infinitesimal version. Quotient objects are also distinguished by a check: $\pi\left(\mathcal{X}_{p}^{\perp}\right)=\check{\mathcal{X}}_{p}^{\perp}, \pi\left(\mathcal{Y}_{p}^{s}\right)=\check{\mathcal{Y}}_{p}^{s} \ldots$

2.7 Remark For each $s$, at each point $p$, the group $\check{H}_{\breve{p}}^{s}$ contains the holonomy group $H\left(\check{\mathcal{Y}}_{\bar{p}}^{s}, \check{g}_{\mid \check{\mathcal{Y}}_{\bar{p}}^{s}}\right)$ of the quotient manifold $\check{\mathcal{Y}}_{\tilde{p}}^{s}$ but in general, it is not equal to it.

\section{3 "Adapted" local coordinates}

To achieve our goal, we build "canonical" coordinates on the reducible-indecomposable Lorentzian manifolds. The choice of such coordinates is divided into three steps.

Step 1. Considering the canonical flag (2.1) and decomposition (2.2) and their integral foliations, the first property it is natural to require from coordinates is the following.

3.1 Definition A coordinate system $\left(x,\left(y^{s}\right)_{s=0}^{k}, z\right)=\left(x,\left(\left(y_{i}^{s}\right)_{i=1}^{n_{s}}\right)_{s=0}^{k}, z\right)$ is called $(\mathcal{X}$, $\left.\left(\mathcal{Y}^{s}\right)_{s=0}^{k}\right)$-foliated if :

- the coordinate $x$ parametrizes the leaves of $\mathcal{X}$,

- the coordinates $\left(x,\left(y_{i}^{s}\right)_{i=1}^{n_{s}}\right)$ parametrize those of $\mathcal{Y}^{s}$, for each $s$ in $\llbracket 0, k \rrbracket$.

It follows from the definition of a foliation and of an atlas of foliation that such systems exist, come the involved foliations from parallel distributions or not. Notice that the coordinates $\left(x,\left(\left(y_{i}^{s}\right)_{i=0}^{n_{s}}\right)_{s=0}^{k}\right)$ parametrize the leaves of $\mathcal{X}^{\perp}$. Besides, restricting possibly their domain, we suppose that the coordinates apply in $\left.I^{n}, I=\right]-\varepsilon, \varepsilon[$ for some $\varepsilon>0$. We investigate here germs of metrics, so we now identify this $I^{n}$ with the manifold $\mathcal{M}$. Before step 2, some observations shall be made.

3.2 Note In [W49, W50], A. G. Walker already proposed "adapted coordinates" on (a wider class of) reducible-indecomposable pseudo-Riemannian manifolds. They are essentially foliated coordinates, with an additional property linked with the first Bianchi identity. They do not suit our purpose: in such coordinates, the metric depends not only on its intrinsic properties but also on the (still too) wide arbitrary choice of the coordinates.

We try here to mimic the case of a Riemannian product i.e. by de Rham's theorem, the case where a holonomy-stable decomposition $\mathrm{T}_{m} \mathcal{M}=\mathbf{E}_{m} \oplus \mathbf{E}_{m}^{\perp}$ exists. Let $\mathcal{E}$ and $\mathcal{E}^{\perp}$ be the corresponding foliations; $\left(\mathcal{E}, \mathcal{E}^{\perp}\right)$-foliated coordinates are product coordinates: once chosen on each factor $\mathcal{E}_{m}$ and $\mathcal{E}_{m}^{\perp}$, they are unique. Here, $\left(\mathcal{X},\left(\mathcal{Y}^{s}\right)_{s=0}^{k}\right)$-foliated coordinates, fixed on $\mathcal{X}_{m}^{\perp} \supset \mathcal{X}_{m}$, are not unique. We add some constraints to achieve this unicity.

3.3 Remark/Notation In $\left(\mathcal{X},\left(\mathcal{Y}^{s}\right)_{s=0}^{k}\right)$-foliated coordinates, it follows from the different orthogonality relations between the involved distributions that the metric $g$ reads

$$
g=\left(\sum_{s=0}^{k} g^{s}\right)+2 \gamma \mathrm{d} z
$$

where $\gamma$ is a 1 -form and where, for each $s$ in $\llbracket 0, k \rrbracket, g^{s}=\sum_{i, j=1}^{n_{s}} g_{i, j}^{s} \mathrm{~d} y_{i}^{s} \mathrm{~d} y_{j}^{s}$.

The matrices $\left(g_{i, j}^{s}\right)_{i, j=1}^{n_{s}}$ depend on the chosen foliated coordinate system but not the corresponding Riemannian metrics $\check{g}^{s}$ defined on each leaf $\check{\mathcal{Y}}_{p}^{s}$ of the foliation $\check{\mathcal{Y}}^{s}$ of $\pi(\mathcal{M})$. On the contrary, those $\check{g}^{s}$ are important canonical objects of the situation. 
Eventually, let us notice the simple but important following property.

3.4 Proposition Let $\left(\mathbf{X},\left(\mathbf{Y}^{s}\right)_{s=0}^{k}\right)$ be some distributions as set out above, except they are not supposed to be parallel, but only integrable. They are parallel if and only if, in $(\mathcal{X}$, $\left.\left(\mathcal{Y}^{s}\right)_{s=0}^{k}\right)$-foliated coordinates, the metric $g$ satisfies:

$$
\left\{\begin{array}{l}
\bullet \text { for each } s \text { in } \llbracket 0, k \rrbracket, \text { the matrix }\left(g_{i, j}^{s}\right)_{i, j=1}^{n_{s}} \text { depends only on } y^{s} \text { and } z \\
\bullet \forall s \in \llbracket 0, k \rrbracket, \quad \mathrm{d} \gamma\left(\mathbf{X}, \mathbf{Y}^{s}\right)=\{0\} \quad \text { and: } \quad \forall r \neq s, \mathrm{~d} \gamma\left(\mathbf{Y}^{r}, \mathbf{Y}^{s}\right)=\{0\}
\end{array}\right.
$$

Proof. Let $\mathbf{A}, \mathbf{B}, \mathbf{C}$ stand for three of the distributions $\left(\mathbf{X},\left(\mathbf{Y}^{s}\right)_{s=0}^{k}\right) ; A, B, C$ stand for coordinate-vectors in $\mathbf{A}, \mathbf{B}, \mathbf{C}$ respectively.

$$
\begin{aligned}
\mathbf{A} \text { is parallel } & \Leftrightarrow \forall A, B, D_{B} A \in \mathbf{A} \text { and } D_{Z} A \in \mathbf{A} \\
& \Leftrightarrow \forall A, B, C, \mathbf{C} \perp \mathbf{A} \Rightarrow g\left(D_{B} A, C\right)=0 \text { and } g\left(D_{Z} A, C\right)=0 \\
\Leftrightarrow \forall A, B, C, \mathbf{C} \perp \mathbf{A} \Rightarrow L_{B} g(A, C)+L_{A} g(B, C)-L_{C} g(A, B)=0 & \quad \text { and } L_{Z} g(A, C)+L_{A} g(Z, C)-L_{C} g(A, Z)=0 .
\end{aligned}
$$

Now $\mathbf{A} \perp \mathbf{C}$ and the coordinates are foliated, so $g(A, C) \equiv 0$. The last equality is then the second point of (3.2); in both cases $\mathbf{A} \perp \mathbf{B}$ or $\mathbf{A}=\mathbf{B}$, the second to last one is the first point of (3.2).

If (3.2) holds, we denote each matrix $\left(g_{i, j}^{s}\right)_{i, j=1}^{n_{s}}$ by $\check{g}_{z}^{s}$, seeing it as a one-parameter family, in $z$, of metrics on $I^{n_{s}}$, identified by the coordinates with plaques of the foliation $\check{\mathcal{Y}}^{s}$. Similarly, for each $z_{0}$, we denote by $\check{g}_{z_{0}}$ the metric on the plaque $\pi\left(\left\{z=z_{0}\right\}\right)$ of the foliation $\check{\mathcal{X}}^{\perp}$. So, $\left(\check{g}_{z}\right)_{z \in I}=\left(\prod_{s=0}^{k} \check{g}_{z}^{s}\right)_{z \in I}$.

Step 2. We choose to prefer $\left(\mathcal{X},\left(\mathcal{Y}^{s}\right)_{s=0}^{k}\right)$-foliated coordinates satisfying the following additional property, which is always possible.

3.5 Definition An $\left(\mathcal{X},\left(\mathcal{Y}^{s}\right)_{s=0}^{k}\right)$-foliated coordinate system $\left(x,\left(y^{s}\right)_{s=0}^{k}, z\right)$ is called here transversally isotropic if $Z=\frac{\partial}{\partial z}$ is $g$-isotropic.

3.6 Notation In foliated, transversally isotropic coordinates, the 1 -form $\gamma$ is given by its restriction $\gamma_{z_{0}}$ to each plaque $\left\{z=z_{0}\right\}$ of $\mathcal{X}^{\perp}$. Then $\left(\gamma_{z}\right)_{z \in I}$ is a 1 -parameter family of 1-forms on $I^{n-1}$.

Step 3. The "adapted" coordinates we use are given by the following theorem. They are centered at some point $m \in \mathcal{M}$; we denote by $m_{z}$ the point of coordinates $(0, \ldots, 0, z)$.

3.7 Theorem (Adapted coordinates) (See also Figure 1 p.8.) (a) There exists, on a neighbourhood of $m$, some $\mathrm{C}^{\infty},\left(\mathcal{X},\left(\mathcal{Y}^{s}\right)_{s=0}^{k}\right)$-foliated, transversally isotropic coordinate systems $\left(x,\left(y^{s}\right)_{s=0}^{k}, z\right)$, centered at $m$ and in which:

(i) along $\mathcal{X}_{m}^{\perp}$ and along the curve $\left(m_{z}\right)_{z}, \gamma=\mathrm{d} x$ (i.e. $\gamma_{0}=\mathrm{d} x$ and $\forall z, \gamma_{z}(0)=\mathrm{d} x$ ),

(ii) for each $z, \gamma_{z}$ is closed,

(iii) setting $\mathcal{S}_{z}^{s}=\mathcal{Y}_{m_{z}}^{s} \cap x^{-1}(\{0\})$, then $\forall s \in \llbracket 1, k \rrbracket, \forall p \in \mathcal{S}_{z}^{s}, \gamma_{\mid \mathrm{T}_{p} \mathcal{S}_{z}^{s}}=0$;

(iv) $g(m)=\left(\sum_{s=0}^{k} \sum_{i=1}^{n_{s}}\left(\mathrm{~d} y_{i}^{s}\right)^{2}\right)+2 \mathrm{~d} x \mathrm{~d} z$ and at every point, $g^{0}=\sum_{i=1}^{n_{0}}\left(\mathrm{~d} y_{i}^{0}\right)^{2}$. 


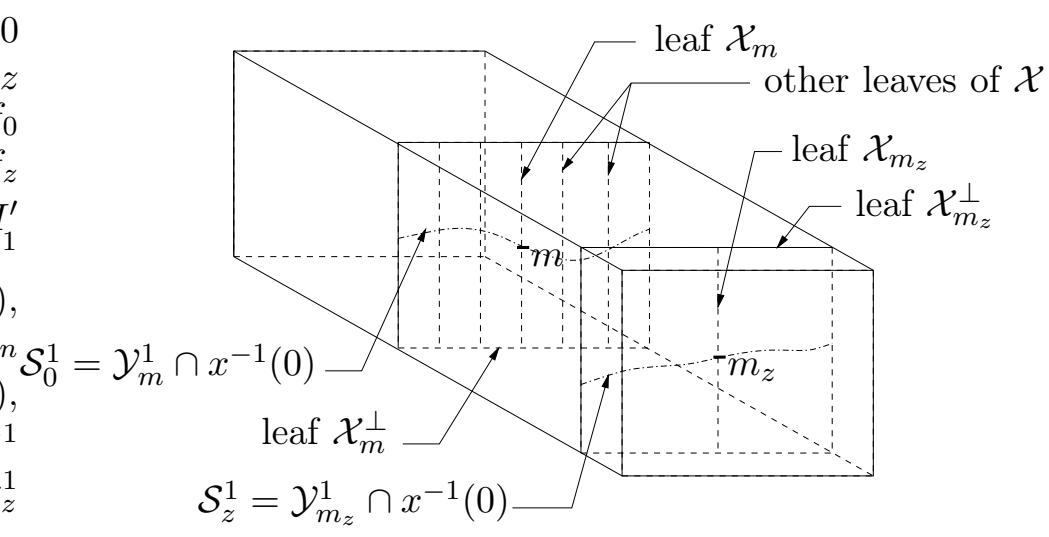

Figure 1: $\mathcal{M}=I^{n}$, case $n=3, n_{0}=0, k=1, \mathbf{X}^{\perp}=\mathbf{Y}^{1}$.

(b) If two such coordinate systems are equal on their submanifolds $\mathcal{S}_{0}^{s}$, for $s \in \llbracket 1, k \rrbracket$, and have equal coordinate vectors $\left(X,\left(Y_{i}^{0}\right)_{i=1}^{n_{0}}\right)$ at $m$, then they are equal where they are both defined. In particular, it is the case if they coincide on $\mathcal{X}_{m}^{\perp}$.

3.8 Remarks - Conversely, as it should be expected, a metric which, in some coordinates $\left(x,\left(y^{s}\right)_{s=0}^{k}, z\right)$, reads as in (3.1) and satisfies (3.2) and the conditions of Theorem 3.7 induces a holonomy representation stabilizing $\operatorname{span}(X), \operatorname{span}\left(X,\left(Y_{i}^{s}\right)_{i=1}^{n_{s}}\right)$ for each $s$ and acting trivially on $\operatorname{span}\left(X,\left(Y_{i}^{0}\right)_{i=1}^{n_{0}}\right) / \operatorname{span}(X)$. This follows from Proposition 3.4 and from a quick checking for the last assertion.

- In adapted coordinates, as $\gamma$ must satisfy (3.2) and the conditions of Theorem 3.7, one checks that $\gamma$ is given by its derivatives $\gamma_{\mid \mathbf{A}}$ along $\mathbf{B}$, for each pair $(\mathbf{A}, \mathbf{B})$ among the distributions $\left(\mathbf{X},\left(\mathbf{Y}^{s}\right)_{s=0}^{k}\right)$ such that $\mathbf{A} \perp \mathbf{B}$ or $\mathbf{A}=\mathbf{B}=\mathbf{Y}^{0}$. Relation (3.3) below provides precisely this data, so in adapted coordinates, it determines $\gamma$.

Finally, adapted coordinates will be useful mainly through both following properties.

3.9 Proposition In adapted coordinates, the form $\gamma$ is such that, for $\mathbf{A}$ and $\mathbf{B}$ any two of the distributions $\left(\mathbf{X},\left(\mathbf{Y}^{s}\right)_{s=0}^{k}\right)$ and for each pair $(A, B)$ of coordinate-vectors in $\mathbf{A} \times \mathbf{B}$,

$$
\left[(\mathbf{A} \perp \mathbf{B}) \text { or }\left(\mathbf{A}=\mathbf{B}=\mathbf{Y}^{0}\right)\right] \Rightarrow \gamma(X) L_{Z}\left(\frac{1}{\gamma(X)} L_{A} \gamma(B)\right)=g(R(A, Z) Z, B) .
$$

For the second property, we need a definition. If $E$ is a fibre bundle with a connection $\nabla$, a differential $\mathrm{d}^{\nabla}$ of any $p$-form with value in $E$ is associated with it, see e.g. [Bes87] p.24. A bilinear form $b$ on $\mathcal{M}$ is a 1 -form with value in $T^{*} \mathcal{M}$, so one defines:

3.10 Definition Let $b$ be a field of bilinear forms on a manifold $\mathcal{M}$ endowed with an affine connection $D$. The $D$-differential $\mathrm{d}^{D}$ of $b$ is defined as

$$
\mathrm{d}^{D} b(U, V, W)=D_{U} b(V, W)-D_{V} b(U, W) .
$$

3.11 Remark If $b$ is symmetric, $\mathrm{d}^{D} b$ satisfies a "Jacobi-" or "Bianchi-type" identity:

$$
\mathrm{d}^{D} b(U, V, W)+\mathrm{d}^{D} b(W, U, V)+\mathrm{d}^{D} b(V, W, U)=0 .
$$

3.12 Proposition If, at $p \in \mathcal{M}, Y, Y^{\prime}$ and $Y^{\prime \prime}$ are in $\mathbf{X}_{p}^{\perp}$, the quantity $g\left(R\left(Y, Y^{\prime}\right) Y^{\prime \prime}, Z\right)$ only depends on the class of $Y, Y^{\prime}$ and $Y^{\prime \prime}$ modulo $\mathbf{X}$, and is invariant by parallel transport 
along the leaf $\mathcal{X}_{p}$. So, if $\left(\check{Y}, \check{Y}^{\prime}, \check{Y}^{\prime \prime}\right)=\pi\left(Y, Y^{\prime}, Y^{\prime \prime}\right)$, the quantity $g\left(R\left(\check{Y}, \check{Y}^{\prime}\right) \check{Y}^{\prime \prime}, Z\right)$ is welldefined. Now in adapted coordinates, at any point $\check{p} \in \pi(\mathcal{M})$, if $\check{Y}, \check{Y}^{\prime}, \check{Y}^{\prime \prime} \in \check{\mathbf{X}}_{\check{p}}^{\perp}$ and with $D_{z}$ the Levi-Civita connection of $\check{g}_{z}$ :

$$
\mathrm{d}^{D z}\left(\frac{\mathrm{d} \check{g}_{z}}{\mathrm{~d} z}\right)\left(\check{Y}, \check{Y}^{\prime}, \check{Y}^{\prime \prime}\right)=-2 g\left(R\left(\check{Y}, \check{Y}^{\prime}\right) \check{Y}^{\prime \prime}, Z\right)
$$

\section{Proof of Theorem 3.7 and of its adjacent results}

Before using them in section 5 to classify the germ of Lorentzian metrics, we build here adapted coordinates on some $\left.\mathcal{M}=I^{n} \subset \mathbb{R}^{n}, I=\right]-\varepsilon, \varepsilon[, \varepsilon>0$, endowed with a reducibleindecomposable Lorentzian metric $g$ of class $C^{\infty}$. We set $m=0^{n} \in I^{n}$ and use the whole previous notation. Each time it is necessary, $\varepsilon$ is implicitly decreased .

A first Lemma sums up the few general basic properties, which we use then steadily, of the degenerate parallel distributions.

4.1 Lemma If a (pseudo-)Riemannian manifold $\mathcal{M}$ admits a parallel distribution $\mathbf{A}$ and if $p \in \mathcal{M}$, for any $A \in \mathbf{A}_{p}$, any $B \in \mathbf{A}_{p}^{\perp}, R(A, B)=0$. For any $B^{\prime} \in \mathbf{A}_{p}^{\perp}, R\left(B, B^{\prime}\right)_{\mid \mathbf{A}_{p}}=0$. Therefore, any vector $A \in \mathbf{A}_{p}$ can be locally extended as a parallel vector field along the integral leaf $\mathcal{A}_{p}^{\perp}$ through $p$ of the distribution $\mathbf{A}^{\perp}$.

If moreover the restricted holonomy group acts trivially on $\mathbf{A}_{p} /\left(\mathbf{A}_{p} \cap \mathbf{A}_{p}^{\perp}\right)$, then for any $A, A^{\prime} \in \mathbf{A}_{p}, R\left(A, A^{\prime}\right)=0$.

Proof. With the same notation, for any $U, V \in \mathrm{T}_{p} \mathcal{M}: g(R(A, B) U, V)=g(R(U, V) A, B)$. As $\mathbf{A}_{p}$ is holonomy-stable, $R(U, V) A \in \mathbf{A}_{p} \perp B$; the first claim follows. With the first Bianchi identity, it gives: $R\left(B, B^{\prime}\right) A=R\left(A, B^{\prime}\right) B+R(B, A) B^{\prime}=0$, so $R\left(B, B^{\prime}\right)_{\mid \mathbf{A}_{p}}=0$.

Let $\mathcal{E}$ be a vector bundle with fibre $E$ over some base $\mathcal{B}$, endowed with an affine connection $\nabla$. By the Ambrose-Singer theorem, the holonomy algebra, at any point $p \in \mathcal{E}$, of $(\mathcal{E}, \nabla)$ is spanned by the $\tau_{\gamma}^{*}\left(R(U, V)_{q}\right)$, where $q$ runs over $\mathcal{B}, U$ and $V$ over the fibre $E_{q}, \gamma$ over the paths from $p$ to $q$ and where $\tau_{\gamma}: E_{p} \rightarrow E_{q}$ is the parallel transport along $\gamma$. We apply this to the bundle with fibre $\mathbf{A}$ along $\mathcal{A}_{p}^{\perp}$ : as all the $R\left(B, B^{\prime}\right)_{\mid \mathbf{A}_{p}}$ vanish, for $q \in \mathcal{A}_{p}^{\perp}$ and $B, B^{\prime} \in \mathrm{T}_{q} \mathcal{A}_{p}^{\perp}=\mathbf{A}_{q}^{\perp}$, the holonomy algebra of that vector bundle is trivial. The next to last claim of the lemma follows. Finally, adding the last assumption, for any $U, V \in \mathrm{T}_{p} \mathcal{M}$, as $R(U, V) \in \mathfrak{h}_{p}, R(U, V) A \subset\left(\mathbf{A}_{p} \cap \mathbf{A}_{p}^{\perp}\right)$, so $g\left(R(U, V) A, A^{\prime}\right)=0$; the last claim follows.

4.2 Remark/Notation Let us now denote by $\pi^{s}$ each projection $\mathcal{M} \supset \mathcal{Y}_{m}^{s} \rightarrow \check{\mathcal{Y}}_{\check{m}}^{s}$. Besides, as we may always, implicitly, decrease $\varepsilon$, thus the size of any domain on which we work, we always consider that if an $f \in C^{r}\left(\mathcal{O}, \mathbb{R}^{N}\right)$ with $\mathcal{O}$ any precompact domain of some $\mathbb{R}^{N^{\prime}}, f$ admits an extension of class $C^{r}$ on some domain $\mathcal{O}^{\prime} \supset \overline{\mathcal{O}}$. This bounds $\|f\|_{C^{r}}$ and hence will give the existence of some Lipschitz constants in further reasonings.

The key lemma, on which Theorem 3.7 is based, is the following. We prove it immediately and then use it to show Theorem 3.7, but you can also admit it in a first time an go directly to Remark 4.8 and Theorem 3.7's proof following it p. 12.

4.3 Lemma In $\mathcal{M}$, let be given a $k$-tuple $\sigma=\left(\sigma^{s}\right)_{s=1}^{k}$ of sections of class $C^{r}$ of the $\left(\pi^{s}\right)_{s=1}^{k}$ and $U, V$ vector fields of class $C^{r}$, defined along $\mathcal{X}_{m}^{\perp}$ and such that:

$$
D_{\mathbf{X}} U \subset \mathbf{X} \text { and } D_{\mathbf{X}} V \subset \mathbf{X} \text { and for any } s, D_{\mathbf{Y}^{s}} U \subset \mathbf{Y}^{s} \text { and } D_{\mathbf{Y}^{s}} V \subset \mathbf{Y}^{s} .
$$


Then there exists on $\mathcal{X}_{m}^{\perp}$ a unique 1 -form $\alpha_{\sigma, U, V}$ such that, at any point $p$, for any $\mathbf{A}, \mathbf{B}$ $\operatorname{among}\left(\mathbf{X},\left(\mathbf{Y}^{s}\right)_{s=0}^{k}\right)$ and any $A \in \mathbf{A}_{p}, B \in \mathbf{B}_{p}$ :

$$
\begin{gathered}
\left(\mathbf{A} \perp \mathbf{B} \text { or } \mathbf{A}=\mathbf{B}=\mathbf{Y}^{0}\right) \Rightarrow D \alpha_{\sigma, U, V}(A, B)=D \alpha_{\sigma, U, V}(B, A)=g(R(A, U) V, B), \\
\alpha_{\sigma, U, V}\left(\mathbf{Y}_{m}^{0}\right)=\{0\} \text { and, for each } s,\left(\sigma^{s}\right)^{*} \alpha_{\sigma, U, V}=0 .
\end{gathered}
$$

Moreover $\alpha_{\sigma, U, V}$ is closed, is of class $C^{r}$ and its components depend multilinearly and continuously, so on a Lipschitzian way, for the $C^{r}$ norms, on $(\sigma, U, V)$. Besides, the holonomy group acts trivially on $\mathbf{X}$ if and only if, for any $(\sigma, U, V), \alpha_{\sigma, U, V}$ is the pull back $\pi^{*}\left(\check{\alpha}_{\sigma, U, V}\right)$ of some closed form $\check{\alpha}_{\sigma, U, V}$ on $\check{\mathcal{M}}$.

Lemma 4.3 follows itself from both Bianchi Identities; the first one through Lemma 4.4 and the second one through Lemma 4.5. Lemma 4.6 provides auxiliary coordinates in which the arguments of the proof of Lemma 4.3 are simplified.

4.4 Lemma At any point $p \in \mathcal{M}$, for any $U, V \in \mathrm{T}_{p} \mathcal{M}$, for any $\mathbf{A}_{p}$ and $\mathbf{B}_{p}$ among $\left(\mathbf{X}_{p},\left(\mathbf{Y}_{p}^{s}\right)_{s=0}^{k}\right)$ and any $A \in \mathbf{A}_{p}$ and $B \in \mathbf{B}_{p}$ :

$$
\left(\mathbf{A}_{p} \perp \mathbf{B}_{p} \text { or } \mathbf{A}_{p}=\mathbf{B}_{p}=\mathbf{Y}_{p}^{0}\right) \Rightarrow g(R(U, A) V, B)=g(R(U, B) V, A) .
$$

Proof. With the notation of the lemma:

$$
\begin{aligned}
g(R(U, A) V, B) & =-g(R(U, A) B, V) \\
& =g(R(A, B) U, V)+g(R(B, U) A, V) \quad \text { (the " first Bianchi Identity"). }
\end{aligned}
$$

Now if $\mathbf{A}_{p} \perp \mathbf{B}_{p}$ or $\mathbf{A}_{p}=\mathbf{B}_{p}=\mathbf{Y}_{p}^{0}, R(A, B)=0$ by Lemma 4.1. So the result.

4.5 Lemma Let $\mathbf{A}$ be a parallel distribution on a (pseudo-)Riemannian manifold $(\mathcal{M}, g)$, $\mathcal{A}_{p}^{\perp}$ be the integral leaf of the distribution $\mathbf{A}^{\perp}$ through some point $p$. Let $A \in \mathbf{A}_{p}$ be a vector, extended as a parallel vector field along $\mathcal{A}_{p}^{\perp}$ (possible by lemma 4.1) and $U$ and $V$ any vector fields along $\mathcal{A}_{p}^{\perp}$ such that $D_{\mathbf{A}^{\perp}} U \subset \mathbf{A}^{\perp}$ and $D_{\mathbf{A}^{\perp}} V \subset \mathbf{A}^{\perp}$. The 1-form defined on $\mathcal{A}_{p}^{\perp}$ by $B \mapsto g(R(U, B), V, A)$ is closed.

If moreover the restricted holonomy group acts trivially on $\mathbf{A}_{p} /\left(\mathbf{A}_{p} \cap \mathbf{A}_{p}^{\perp}\right)$, all claims hold also with $\mathbf{A}^{\perp}$ and $\mathcal{A}_{p}^{\perp}$ replaced by $\mathbf{A}+\mathbf{A}^{\perp}$ and the integral leaf of it through $p$.

Proof. It follows from the "second Bianchi Identity". It is sufficient to work, at each point $q \in \mathcal{A}_{p}^{\perp}$, with two normal coordinate-vector fields $B$ and $B^{\prime}$; at $q$ : $D_{B} B^{\prime}=D_{B^{\prime}} B=0$.

$$
\begin{aligned}
& L_{B}\left(g\left(R\left(U, B^{\prime}\right) V, A\right)\right) \\
&= g\left(D_{B} R\left(U, B^{\prime}\right) V, A\right)+g\left(R\left(D_{B} U, B^{\prime}\right) V, A\right)+g\left(R\left(U, B^{\prime}\right) D_{B} V, A\right) \\
&= g\left(D_{B^{\prime}} R(U, B) V, A\right)+g\left(D_{U} R\left(B, B^{\prime}\right) V, A\right) \\
&+g\left(R\left(D_{B} U, B^{\prime}\right) V, A\right)+g\left(R(U, A) D_{B} V, B^{\prime}\right) \\
&(\text { The Bianchi identity for the first term, Lemma } 4.4 \text { for the last one }) \\
&=g\left(D_{B^{\prime}} R(U, B) V, A\right)-g\left(D_{U} R\left(B, B^{\prime}\right) A, V\right) \\
&-g\left(R\left(D_{B} U, B^{\prime}\right) A, V\right)-g\left(R\left(D_{B} V, B^{\prime}\right) A, U\right) .
\end{aligned}
$$

Now by Lemma 4.1, as both last terms are in $g\left(R\left(\mathbf{A}^{\perp}, \mathbf{A}^{\perp}\right) \mathbf{A}, \cdot\right)$, they vanish; for the same reason, so does the second term (as $\mathbf{A}$ and $\mathbf{A}^{\perp}$ are parallel, $D_{U} R\left(\mathbf{A}^{\perp}, \mathbf{A}^{\perp}\right) \mathbf{A}$ also vanishes). By symmetry, $L_{B}\left(g\left(R\left(U, B^{\prime}\right) V, A\right)\right)=L_{B^{\prime}}(g(R(U, B) V, A))$ and we are done. The last claim follows from the same calculations together with the last claim of Lemma 4.1. 
4.6 Lemma (i) $\mathcal{X}_{m}^{\perp} \subset \mathcal{M}$ admits a $\left(\mathcal{X},\left(\mathcal{Y}^{s}\right)_{s=0}^{k}\right)$-foliated coordinate system $\left(\bar{x},\left(\bar{y}^{s}\right)_{s=1}^{k}\right)=$ $\left(\bar{x},\left(\left(\bar{y}_{i}^{s}\right)_{i=1}^{n_{s}}\right)_{s=1}^{k}\right)$, of class $C^{\infty}$, such that, for any $(r, s)$, any $i \leqslant n_{s}$ and $j \leqslant n_{r}$ :

$$
\begin{gathered}
D_{\bar{X}} \bar{Y}_{i}^{s}=D_{\bar{Y}_{i}^{s}} \bar{X}=0 \quad \text { and }(r \neq s \text { or } r=s=0) \Rightarrow D_{\bar{Y}_{i}^{s}} \bar{Y}_{j}^{r}=0, \\
\text { at } m, g\left(\bar{Y}_{i}^{s}, \bar{Y}_{j}^{r}\right)=0 \text { if }(s, i) \neq(r, j) \text { and } g\left(\bar{Y}_{i}^{s}, \bar{Y}_{i}^{s}\right)=1 .
\end{gathered}
$$

(ii) $\mathcal{M}$ admits a $\left(\mathcal{X},\left(\mathcal{Y}^{s}\right)_{s=0}^{k}\right)$-foliated coordinate system $\left(\bar{x},\left(\bar{y}^{s}\right)_{s=1}^{k}, z\right)=\left(\bar{x},\left(\left(\bar{y}_{i}^{s}\right)_{i=1}^{n_{s}}\right)_{s=1}^{k}\right.$, $z$ ), of class $C^{\infty}$, satisfying (4.5) and (4.6).

Proof. (i) It follows immediately from Lemma 4.1. For clarity, let us however detail the construction. By Lemma 4.1, we may choose a parallel vector field $0 \neq \bar{X} \in \mathbf{X}$ on $\mathcal{X}_{m}^{\perp}$. Take, on a neighbourhood of $\check{m} \in \check{\mathcal{X}}_{\check{m}}^{\perp} \simeq \prod_{s} \check{\mathcal{Y}}_{\check{m}}^{s}$, a product coordinate system $\left(\check{\bar{y}}^{s}\right)_{s=1}^{k}$. As $\check{\mathcal{Y}}_{\check{m}}^{0}$ is flat, one can choose it such that the $\check{\bar{y}}_{i}^{0}$ are affine coordinates, i.e. such that the $\check{\bar{Y}}_{i}^{0}$ are parallel. For each $s$, take then any section $\sigma^{s}$ of $\pi^{s}: \mathcal{Y}_{m}^{s} \rightarrow \check{\mathcal{Y}}_{\tilde{m}}^{s}$; for $s=0$, take $\sigma^{0}$ affine, i.e. such that $D_{\left(\sigma^{0}\right)_{*} \check{Y}_{i}^{0}}\left(\sigma^{0}\right)_{*} \check{Y}_{j}^{0}=0$, which is possible as the holonomy group of $\mathcal{X}_{m}^{\perp}$ acts trivially on $\mathbf{Y}_{m}^{0}$. Consider the vector fields $\bar{Y}_{i}^{s}=\left(\sigma^{s}\right)_{*} \check{\bar{Y}}_{i}^{s}$, defined along the tangent bundle $\mathrm{T} \mathcal{S}^{s}$ of the image $\mathcal{S}^{s}$ of each $\sigma^{s}$. By construction, $\mathcal{S}^{s}$ intersects each leaf of the integral foliation $\mathcal{Y}^{s \perp}$ of the distribution $\mathbf{Y}^{s \perp}=+_{r \neq s} \mathbf{Y}^{r}$ in exactly one point. Thus, at each such point $p$, by Lemma $4.1,\left(\bar{Y}_{i}^{s}\right)_{p}$ is extended as a parallel vector field $\bar{Y}_{i}^{s}$ along $\mathcal{Y}_{p}^{s \perp}$. This provides fields $\bar{Y}_{i}^{s}$ on the whole chosen neighbourhood of $m$. They commute, as $D$ is torsion free: for $r \neq s$, by definition, $D_{\bar{Y}_{j}^{r}} \bar{Y}_{i}^{s}=0$, so $\left[\bar{Y}_{j}^{r}, \bar{Y}_{i}^{s}\right]=0$. Along $\mathcal{S}^{s}$, the $\bar{Y}_{i}^{s}$ are coordinate-vector fields for the coordinates $\left(\sigma^{s}\right)^{*}\left(\check{y}^{s}\right)$ so they commute. Finally, for $A$ any vector among $\left(\bar{X},\left(\left(\bar{Y}_{i}^{r}\right)_{i=1}^{n_{r}}\right)_{r \neq s}\right): D_{A}\left[\bar{Y}_{i}^{s}, \bar{Y}_{j}^{s}\right]=D_{A} D_{\bar{Y}_{i}^{s}} \bar{Y}_{j}^{s}-D_{A} D_{\bar{Y}_{j}^{s}} \bar{Y}_{i}^{s}$. Now: $D_{A} D_{\bar{Y}_{i}^{s}} \bar{Y}_{j}^{s}=$ $R\left(A, \bar{Y}_{i}^{s}\right) \bar{Y}_{j}^{s}+D_{\bar{Y}_{i}^{s}} D_{A} \bar{Y}_{j}^{s}+D_{\left[A, \bar{Y}_{i}^{s}\right]} \bar{Y}_{j}^{s}=0 ; R\left(A, \bar{Y}_{i}^{s}\right) \bar{Y}_{j}^{s}=0$ by the first point of Lemma 4.1, $D_{A} \bar{Y}_{j}^{s}=0$ by construction and we have just shown that $\left[A, \bar{Y}_{i}^{s}\right]=0$. Symmetrically, $D_{A} D_{\bar{Y}_{j}^{s}} \bar{Y}_{i}^{s}=0$ so $D_{A}\left[\bar{Y}_{i}^{s}, \bar{Y}_{j}^{s}\right]=0$; so $\left[\bar{Y}_{i}^{s}, \bar{Y}_{j}^{s}\right] \equiv 0$ and we are done. The induced integral coordinates $\left(\bar{x},\left(\bar{y}^{s}\right)_{s=0}^{k}\right)$ are as wished; they are immediately of class $C^{\infty}$.

(ii) Choose any regular path $\left(m_{z}\right)_{z \in I}$, transverse to the leaves of $\mathcal{X}_{m}^{\perp}$ and with $m_{0}=m$; this gives the last coordinate, set constant, equal to $z$, on each plaque $\mathcal{X}_{m_{z}}^{\perp}$. Choose then, for each $s$, a regular family $\left(\sigma_{z}^{s}\right)_{z \in I}$ of sections of $\pi_{z}^{s}: \mathcal{Y}_{m_{z}}^{s} \rightarrow \check{\mathcal{Y}}_{\tilde{m}_{z}}^{s}$ and apply point (i).

4.7 Remark It follows from the proof of Point (i) that a coordinate system as in (i) is uniquely determined by the choice of the basis $\left(\bar{X},\left(\bar{Y}_{i}^{0}\right)_{i=1}^{n_{0}}\right)$ of $\mathrm{T}_{m} \mathcal{M}$ and of the sections $\left(\sigma^{s}\right)_{s=1}^{k}$ of the $\pi^{s}: \mathcal{Y}_{m}^{s} \rightarrow \check{\mathcal{Y}}_{\tilde{m}}^{s}$. These basis and sections may be chosen arbitrarily.

Moreover, the reader can check that coordinates of $\mathcal{X}_{m}^{\perp}$ as in (i) satisfy exactly what is required from adapted coordinates by Theorem 3.7, in restriction to $\mathcal{X}_{m}^{\perp}$.

So, coordinates of $\mathcal{X}_{m}^{\perp}$ as in (i) range all possible values of adapted coordinates (if such exist), restricted to $\mathcal{X}_{m}^{\perp}$. This remark will be important in the proof of Theorem 3.7.

Proof of Lemma 4.3. Let $\left(\sigma^{s}\right)_{s=1}^{k}$ be the sections given in the statement of Lemma 4.3, and $\sigma^{0}$ any affine section of $\pi^{0}$ i.e. such that $\sigma^{0}\left(\check{\mathcal{Y}}^{0}\right)$ is totally geodesic, flat, in $\mathcal{X}_{m}^{\perp}$ (possible as the holonomy group of $\mathcal{X}_{m}^{\perp}$ acts trivially on $\mathbf{Y}_{m}^{0}$ ). Lemma 4.6 (i) provides coordinates $\left(\bar{x},\left(\bar{y}^{s}\right)_{s=0}^{k}\right)$ satisfying (4.5) and (4.6) and such that $\sigma^{s}\left(I^{n_{s}}\right)=\cap_{r \neq s}\left\{\bar{y}^{r}=0\right\} \cap\{\bar{x}=0\}$. To build $\alpha_{\sigma, U, V}$, let us build the functions $\alpha_{\sigma, U, V}(X)$ and, for each $s \in \llbracket 0, k \rrbracket$ and $i \leqslant n_{s}$, the functions $\alpha_{\sigma, U, V}\left(\bar{Y}_{i}^{s}\right)$, respectively denoted by $f_{0}^{0}$ and $f_{i}^{s}$.

- On the one hand, let us define, on each leaf of the integral foliation $\mathcal{Y}^{s \perp}$ of the distribution $\mathbf{Y}^{s \perp}$, for $s \geqslant 1$, and on $\mathcal{X}_{m}^{\perp}$, for $s=0$, the 1 -form $\theta_{i}^{s}=g\left(R\left(\bar{Y}_{i}^{s}, U\right) V, \cdot\right)$, with 
conventionally $\theta_{0}^{0}=g(R(X, U) V, \cdot)$. We recall that the $\bar{Y}_{i}^{s}$ are the coordinate-vectors associated with the $\left(\bar{y}_{i}^{s}\right)_{i, s}$. Each $\theta_{i}^{s}$ is closed by Lemma 4.5. As the $\left(\bar{x},\left(\bar{y}_{i}^{s}\right)_{i, s}\right)$ satisfy (4.5), for $r \neq s$ or $r=s=0, D \alpha_{\sigma, U, V}\left(\bar{Y}_{j}^{r}, \bar{Y}_{i}^{s}\right)=L_{\bar{Y}_{j}^{r}} \alpha_{\sigma, U, V}\left(\bar{Y}_{i}^{s}\right)=\mathrm{d} f_{i}^{s} . \bar{Y}_{j}^{r}$. Besides, by Lemma 4.4, $\theta_{i}^{s}\left(\bar{Y}_{j}^{r}\right)=\theta_{j}^{r}\left(\bar{Y}_{i}^{s}\right)$. Thus $\alpha_{\sigma, U, V}$ satisfies (4.2) if and only if:

$$
\left\{\begin{array}{l}
\forall s \geqslant 1, \forall i \in \llbracket 1, n_{s} \rrbracket, \mathrm{d} f_{i \mid \mathbf{Y}^{s \perp}}^{s}=\theta_{i}^{s} \\
\forall i \in \llbracket 0, n_{s} \rrbracket, \mathrm{d} f_{i}^{0}=\theta_{i}^{0}
\end{array}\right.
$$

- On the other hand, $\alpha_{\sigma, U, V}$ satisfies (4.3) if and only if, for $i \in \llbracket 0, n_{0} \rrbracket, f_{i}^{0}=0$ at $m$ and, for each $s \geqslant 1, i \leqslant n_{s}, f_{i}^{s}=0$ on $\mathcal{S}^{s}=\mathcal{Y}_{m}^{s} \cap x^{-1}(0)$. Now each $\mathcal{S}^{s}$, for $s \geqslant 1$, intersects each leaf of $\mathcal{Y}^{s \perp}$ in exactly one point, so, as all $\theta_{i}^{s}$ are closed, Equations (4.7) determine the $f_{i}^{s}, s \geqslant 0$, in a unique way. This defines $\alpha_{\sigma, U, V}$.

Each $f_{i}^{s}$ is of class $C^{r}$ : along the leaves of $\mathcal{Y}^{s \perp}$, or along $\mathcal{X}_{m}^{\perp}$, for $s=0$, it is the integral of a closed 1-form of class $C^{r}$ (so it seems to be $C^{r+1}$, but the sections $\sigma^{s}$ and hence the coordinates $\left(\bar{x},\left(\bar{y}^{s}\right)_{s}\right)$ are only of class $\left.C^{r}\right)$, transversely to them, it is also $C^{r}$ as the functions $\theta_{i}^{s}\left(\bar{Y}_{j}^{r}\right)$ are $C^{r}$ (in all directions). So $\alpha_{\sigma, U, V}$ is of class $C^{r}$.

Besides, the integration of closed 1-forms, with prescribed initial condition, is a linear continuous operator from the set of the functions of class $C^{r}$ into itself (from $C^{r}$ into $C^{r+1}$ actually, but the coordinates are $C^{r}$ ), so $\alpha_{\sigma, U, V}$ depends linearly and continuously for the $C^{r}$ norm, on the $\theta_{i}^{s}$, i.e. on the fields $U$ and $V$. Eventually, a possible change of the sections $\sigma^{s}$ induces a coordinate change $\bar{x} \rightsquigarrow \Phi\left(\left(\sigma^{s}\right)_{s=1}^{k}\right)(\bar{x}), \Phi$ being an affine and continuous fonction of the $\left(\sigma^{s}\right)_{s=1}^{k}$, for the $C^{r}$ norms. So $\alpha_{\sigma, U, V}$ depends multilinearly and continuously (so is Lipschitzian), for the $C^{r}$ norms, on $(\sigma, U, V)$.

To check that $\mathrm{d} \alpha_{\sigma, U, V}=0$, it remains to check that, for $s \geqslant 1$, the $\mathrm{d} \alpha_{\sigma, U, V}\left(\bar{Y}_{i}^{s}, \bar{Y}_{j}^{s}\right)$ vanish. It holds on $\mathcal{S}^{s}$. So, taking any $r \neq s$ and $l \leqslant n_{r}$, let us check that $L_{\bar{Y}_{l}^{r}} \mathrm{~d} \alpha_{\sigma, U, V}\left(\bar{Y}_{i}^{s}, \bar{Y}_{j}^{s}\right)=$ 0. This is immediate: $L_{\bar{Y}_{l}^{r}} L_{\bar{Y}_{i}^{s}} \alpha_{\sigma, U, V}\left(\bar{Y}_{j}^{s}\right)=L_{\bar{Y}_{i}^{s}} L_{\bar{Y}_{l}^{r}} \alpha_{\sigma, U, V}\left(\bar{Y}_{j}^{s}\right)=L_{\bar{Y}_{i}^{s}} L_{\bar{Y}_{j}^{s}} \alpha_{\sigma, U, V}\left(\bar{Y}_{l}^{r}\right)=$ $L_{\bar{Y}_{j}^{s}} L_{\bar{Y}_{i}^{s}} \alpha_{\sigma, U, V}\left(\bar{Y}_{l}^{r}\right)$; this expression being symmetric in $i$ and $j$, we are done.

The last claim of Lemma 4.3 follows easily from the Ambrose-Singer theorem.

4.8 Remark So, because of the Bianchi identities, the bilinear form $g(R(\cdot, U) V, \cdot)$ can be "integrated" in the sense of Lemma $4.3 ; \alpha_{\sigma, U, V}$ is this integral. This holds for any pseudoRiemannian metric $g$ the holonomy group of which preserves a totally degenerate space $\mathbf{X}$ and acts totally reducibly on $\mathbf{X}^{\perp} / \mathbf{X}$. Now we will use that $\operatorname{dim} \mathbf{X}=1$.

Proof of Theorem 3.7. The coordinate systems of Theorem 3.7 appear as solutions of an O.D.E. in an open subset $\mathcal{G}$ of some (infinite dimensional) Banach space $\mathcal{F}$. To simplify the reasoning, let us first parametrize $\mathcal{M}=I^{n}$ with coordinates $\left(\bar{x},\left(\bar{y}^{s}\right)_{s=0}^{k}, \bar{z}\right)$ of the type provided by Lemma 4.6 ; such a parametrization is in particular $\left(\mathcal{X},\left(\mathcal{Y}^{s}\right)_{s=0}^{k}\right)$-foliated, so at any point, $\mathbf{X}=\operatorname{span}(\bar{X})$ and $\mathbf{Y}^{s}=\operatorname{span}\left(\bar{X},\left(\bar{Y}_{i}^{s}\right)_{i=1}^{n_{s}}\right)$. We identify $\mathcal{M}$ and $I^{n}$ through these coordinates. In particular, the leaves of $\mathcal{X}^{\perp}$ are the hypersurfaces $\left\{\bar{z}=\bar{z}_{0}\right\}$, we denote each such leaf by $\mathcal{X}_{\bar{z}_{0}}^{\perp}$. The leaves of $\mathcal{X}^{\perp}$ are parametrized by $\bar{x}$, so the projection $\pi: \mathcal{M} \mapsto \mathcal{M} / \mathcal{X}$ is simply the dropping of the first coordinate: $I^{n} \rightarrow I^{n-1}$. Let us take a copy of $\left(\frac{1}{2} I\right)^{n-1}$, with the canonical coordinates denoted by $\left(x,\left(\left(y_{i}^{s}\right)_{i=1}^{n_{s}}\right)_{s=0}^{k}\right)$ and let us introduce:

$$
\mathcal{F}=\left\{f \in C^{r}\left(\left(\frac{1}{2} I\right)^{n-1}, I^{n-1}\right) ; \forall s \leqslant k, \forall i \leqslant n_{s}, \frac{\partial f}{\partial y_{i}^{s}} \in \mathbf{Y}^{s}\right\},
$$

this is a closed subspace of the Banach space $C^{r}\left(\left(\frac{1}{2} I\right)^{n-1}, I^{n-1}\right)$. Then we introduce

$$
\mathcal{G}=\{f \in \mathcal{F} ; f \text { is a diffeomorphism on its image }\},
$$


which is an open subset of $\mathcal{F}$, for the $C^{r}$ norm. A foliated parametrization of an open subset of $I^{n}$ (of class $C^{r}$ and, transversely to the leaves of $\mathcal{X}^{\perp}$, of class $C^{\infty}$ ) is given by a pair $\left(\left(f_{z^{\prime}}\right)_{z^{\prime} \in I^{\prime}}, \zeta\right)$ with $\left(f_{z^{\prime}}\right)_{z^{\prime} \in I^{\prime}}$ a curve of class $C^{\infty}$ in $\mathcal{G}$ and $\zeta$ a $C^{\infty}$-diffeomorphism from $I^{\prime}$ onto $I$, with $I^{\prime}$ some interval. Indeed, such a pair gives an embedding $F_{f, \zeta}:\left(\frac{1}{2} I\right)^{n-1} \times I^{\prime} \rightarrow I^{n}$ defined by $F_{f, \zeta}\left(x,\left(y^{s}\right)_{s=1}^{k}, z\right)=\left(f_{z}\left(x,\left(y^{s}\right)_{s=1}^{k}\right), \zeta(z)\right)$.

4.9 Remark In fact, we look for an adapted parametrization of a neighbourhood of $m$, such as defined in Theorem 3.7. So, by Remark 4.7, to prove Theorem 3.7, we are led back to show the existence (Th. 3.7 (a)) and uniqueness (Th. 3.7 (b)), of adapted coordinates, equal on $\mathcal{X}_{m}^{\perp}$ to the fixed $\left(\bar{x},\left(\bar{y}^{s}\right)_{s=1}^{k}\right)$, given by Lemma 4.6. So in the following we suppose, without loss of generality, that $f_{0}$ is the identity embedding $\left(\frac{1}{2} I\right)^{n-1} \rightarrow I^{n-1}$.

Besides, by the following lemma, we get rid of the determination of $\zeta$ and have only to focus on $\left(f_{z}\right)_{z \in I^{\prime}}$. The (easy) proof is postponed to the end of the proof of the Theorem.

4.10 Lemma Let $\left(x,\left(y^{s}\right)_{s=0}^{k}, z\right)$ be an adapted system of coordinates, centered at $m$. Then the curve $m_{z}$ parametrized by $(0, \ldots, 0, z)$ is a geodesic. In particular, as the tangent vector $Z$ to this geodesic is, at $m$, determined by: $g(Z, X)=1, \forall s, i, g\left(Z, Y_{i}^{s}\right)=0$ and $g(Z, Z)=0$, the coordinate $z$ is determined by the data of the basis $\left(X,\left(\left(Y_{i}^{s}\right)_{i=1}^{n_{s}}\right)_{s=0}^{k}\right)$ of $\mathbf{X}_{m}^{\perp}$.

Therefore, after a possible reparametrization, we suppose that the coordinate $\bar{z}$ of $I^{n}$ is such that $\bar{z} \mapsto(0, \ldots, 0, \bar{z})$ is a geodesic; $I^{\prime} \subset I$ and $\zeta$ is the identity embedding $I^{\prime} \rightarrow I$.

Now let $f=\left(f_{z}\right)_{z \in I}$ be a curve of class $C^{\infty}$ in $\mathcal{G}$ with $f_{0}$ the identity embedding $\left(\frac{1}{2} I\right)^{n-1} \rightarrow I^{n-1}$; we associate some objects with it.

- $F_{f}:\left(x,\left(y^{s}\right)_{s=1}^{k}, z\right) \mapsto\left(f_{z}\left(x,\left(y^{s}\right)_{s=1}^{k}\right), z\right)$ is a foliated parametrization of some open subset of $\mathcal{M}=I^{n}$ - we have now dropped $\zeta$.

- For each value $z_{0}$ of $z$, with $f_{z_{0}}^{\prime}=\left(\frac{\mathrm{d}}{\mathrm{d} z} f\right)_{\mid z_{0}} \in T \mathcal{G}=\mathcal{F}$ is associated the vector field $Z_{\mid z_{0}}$ defined along $f_{z_{0}}\left(\left(\frac{1}{2} I\right)^{n-1}\right) \times\left\{z_{0}\right\}$ by $Z_{\mid z_{0}}\left(f_{z_{0}}(p)\right)=\left(f_{z_{0}}^{\prime}(p), 1\right) ; Z_{\mid z_{0}}$ is the last coordinate-vector field of $F_{f}$, along $\left(f_{z_{0}}, z_{0}\right)$.

- Along the whole image of $F_{f}$, this defines a vector field $Z$; for each value $z_{0}$ of $z$, its covariant derivative $\left(D_{Z} Z\right)_{\mid z_{0}}$, which is, as well as $Z_{\mid z_{0}}$, a vector field along $\left(f_{z_{0}}, z_{0}\right)$, depends only on $f_{z_{0}}, f_{z_{0}}^{\prime}, f_{z_{0}}^{\prime \prime}$ and $z_{0}$, through the Christoffel symbols of the metric $g$ along $\left(f_{z_{0}}, z_{0}\right)$; this dependence is lipschitzian for the $C^{r}$ norm. In the converse sense, $f_{z_{0}}^{\prime \prime}$ is also determined, on a Lipschitzian way for the $C^{r}$ norm, by the quadruple $\left(f_{z_{0}}, f_{z_{0}}^{\prime},\left(D_{Z} Z\right)_{\mid z_{0}}, z_{0}\right)$.

Besides, for each value $z_{0}$ of $z$, the hypersurface $\left.f_{z_{0}}\left(\{0\} \times\left(\frac{1}{2} I\right)^{n-2}\right)\right)$ of $I^{n-1}$ is transverse to the first factor $I$, i.e. to the leaves of $\mathcal{X}$, i.e. to the fibres of $\pi: \mathcal{X} \perp z_{0}^{\perp} \rightarrow \mathcal{X}_{z_{0}}^{\perp} / \mathcal{X}$. So, for each $s \geqslant 1$, a section $\sigma^{s}\left(f_{z_{0}}\right)$ of $\pi_{z_{0}}^{s}: \mathcal{Y}_{m_{z_{0}}}^{s} \rightarrow \mathcal{Y}_{m_{z_{0}}}^{s} / \mathcal{X}$ is associated with $f_{z_{0}}$; more precisely, $\sigma^{s}\left(f_{z_{0}}\right)$ is defined on the image of $\pi_{z_{0}}^{s} \circ f_{z_{0}}$. Figure 2 above gives a drawing in low dimension. Moreover, as $\left(f_{z}\right)_{z \in I^{\prime}}$ is a curve in $\mathcal{G}$, its derivative $\left(f_{z}^{\prime}\right)_{z \in I^{\prime}}$ is in $T \mathcal{G}=\mathcal{F}$ so the associated vector field $Z$ satisfies (4.1) — both conditions are equivalent, it comes from the fact that the distributions $\mathbf{X}$ and $\mathbf{Y}^{s}$ are parallel. Therefore, with $\left(f_{z_{0}}, f^{\prime}\left(z_{0}\right), z_{0}\right)$ is associated the one-form $\alpha_{\sigma\left(f_{z_{0}}\right), Z, Z}$ given by Lemma 4.3. We denote it by $\alpha\left(f_{z_{0}}, f_{z_{0}}^{\prime}, z_{0}\right)$.

Now Theorem 3.7 is based on Remark 4.9 and on both following lemmas, the first of which is already nearly proven by all that precedes.

4.11 Lemma There exists, for small values of $|z|$, a unique curve $\left(f_{z}\right)_{z}$ in $\mathcal{G}$ such that:

- for all $z_{0},\left\{\begin{array}{l}D_{Z} Z \equiv \alpha\left(f_{z_{0}}, f_{z_{0}}^{\prime}, z_{0}\right)^{\sharp}[\mathbf{X}] \\ g\left(D_{Z} Z, Z\right)=0\end{array}\right.$, 


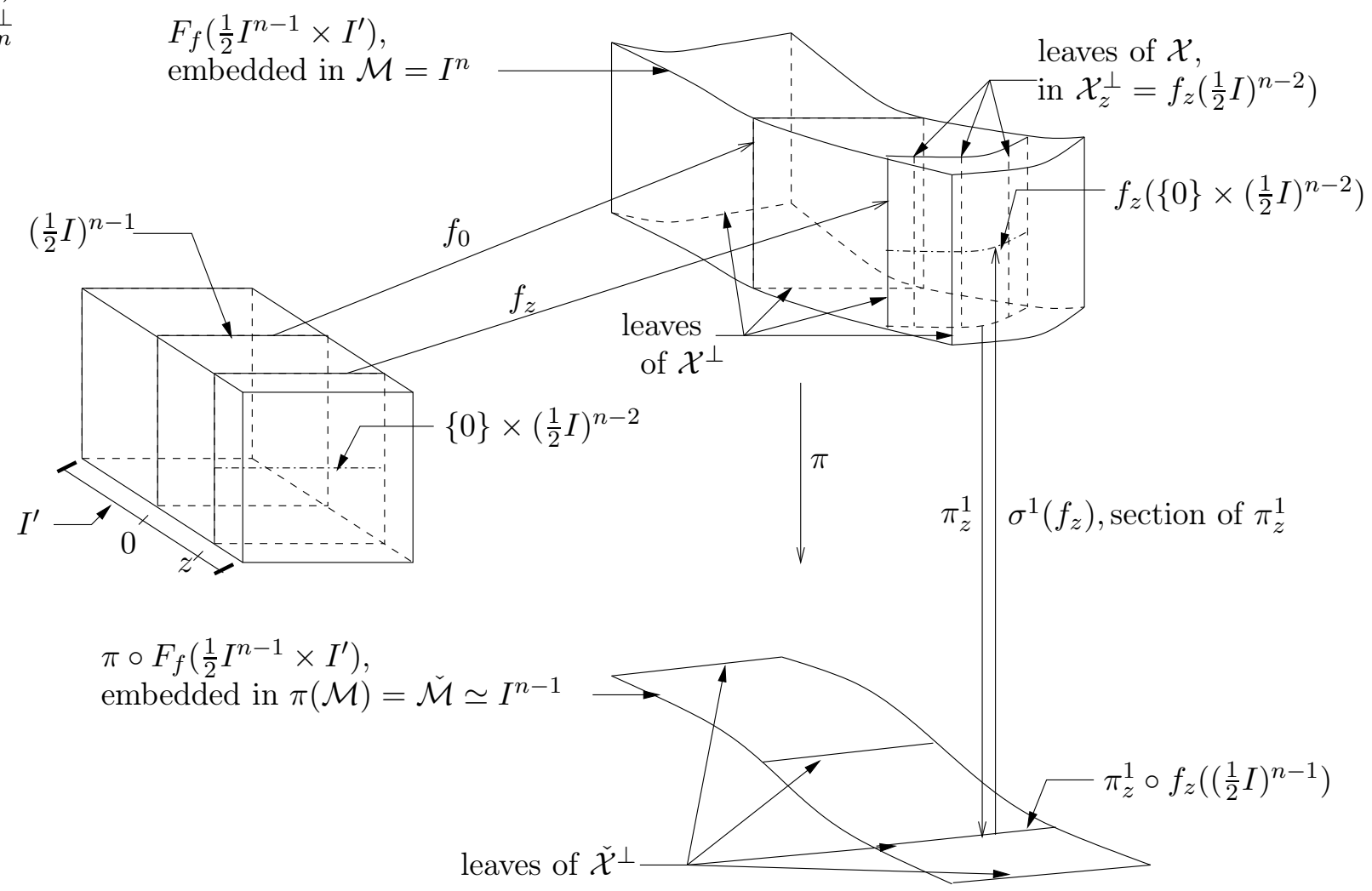

Figure 2: definition of the $\sigma^{s}$, drawing with $n=3, n_{0}=0, k=1, \mathbf{X}^{\perp}=\mathbf{Y}^{1}$.

- at $z=0, f_{0}$ is the identity embedding of $\left(\frac{1}{2} I\right)^{n-1}$ in $I^{n-1}$ and $Z_{\mid z=0}$, thus $f_{0}^{\prime}$, is defined along $f_{0}$ by: $g\left(Z_{\mid 0}, X\right)=1 ; \forall s, i, g\left(Z_{\mid 0}, Y_{i}^{s}\right)=0$ and $g\left(Z_{\mid 0}, Z_{\mid 0}\right)=0$.

4.12 Lemma Let $\left(\bar{x},\left(\bar{y}^{s}\right)_{s=0}^{k}\right)$ be some coordinates of a neighbourhood of $m$ in $\mathcal{X}_{m}^{\perp}$, as given in Lemma 4.6, (i). A system of foliated coordinates of $\mathcal{M}$, given as a curve $\left(f_{z}\right)_{z \in I^{\prime}}$ in $\mathcal{G}$, is adapted, as defined in Theorem 3.7, and, in restriction to $\mathcal{X}_{m}^{\perp}$, equal to $\left(\bar{x},\left(\bar{y}^{s}\right)_{s=0}^{k}\right)$, if and only if it satisfies the conditions of Lemma 4.11.

The lemmas' proofs are postponed. Let us end the Theorem's proof. For each $r \geqslant 3$, both last lemmas give the existence of an adapted system of coordinates of a neighbourhood of $m$, of class $C^{r}$ (and, transversely to the leaves of $\mathcal{X}^{\perp}$, of class $C^{\infty}$ ). They give also the unicity of such a system, once it is fixed on $\mathcal{X}_{m}^{\perp}$. Hence in particular, for a given initial value of class $C^{\infty}$ on $\mathcal{X}_{m}^{\perp}$, the solutions in each space $C^{r}$ coincide: this unique solution is thus $C^{r}$ for all $r$, i.e. $C^{\infty}$ and Theorem 3.7 is proven. We are left with proving Lemmas 4.10, 4.11 and 4.12 .

Proof of Lemma 4.10. Let $\left(x,\left(y^{s}\right)_{s=0}^{k}, z\right)$ be an adapted coordinate system centered at $m$. Let us show that $\left(m_{z}\right)_{z}$ is a geodesic. By theorem 3.7 (a) (i), along $\left(m_{z}\right)_{z}, \gamma=\mathrm{d} x$ i.e. $g(Z, X)=0$ and for all $s, i, g\left(Z, Y_{i}^{s}\right)=0$. Thus along $\left(m_{z}\right)_{z}, g\left(D_{Z} Z, X\right)=L_{Z} g(Z, X)-$ $\frac{1}{2} L_{X} g(Z, Z)=0$, as $g(Z, Z)=0$ everywhere; similarly, for all $s, i, g\left(D_{Z} Z, Y_{i}^{s}\right)=0$. Finally as $g(Z, Z)$ vanishes everywhere, $g\left(D_{Z} Z, Z\right)$ too, so along $\left(m_{z}\right)_{z}, D_{Z} Z=0$.

Proof of Lemma 4.11. Let us clarify the meaning of " $\equiv \alpha\left(f_{z_{0}}, f_{z_{0}}^{\prime}, z_{0}\right)^{\sharp}[\mathbf{X}]$ ": the 1-form $\alpha\left(f_{z_{0}}, f_{z_{0}}^{\prime}, z_{0}\right)$ is defined along $\mathcal{X}_{z_{0}}^{\perp}$, so $\alpha\left(f_{z_{0}}, f_{z_{0}}^{\prime}, z_{0}\right)^{\sharp}$, given by the musical isomorphism 
induced by $g$, is, along $\mathcal{X}_{z_{0}}^{\perp}$, a section of $\mathrm{T} \mathcal{M} /\left(\mathrm{T} \mathcal{X}_{z_{0}}^{\perp}\right)=\mathrm{T} \mathcal{M} / \mathbf{X}$; in other terms, it is a vector field defined modulo $\mathbf{X}$. As, by construction, $Z$ is never orthogonal to $\mathbf{X}$, both conditions of the system (4.8) define $\left(D_{Z} Z\right)_{\mid z_{0}}$ as a function of $\alpha\left(f_{z_{0}}, f_{z_{0}}^{\prime}, z_{0}\right)$ and $Z_{\mid z_{0}}$. As, in turn, $\alpha\left(f_{z_{0}}, f_{z_{0}}^{\prime}, z_{0}\right)$ is a Lipschitzian function of its arguments, for the $C^{r}$ norms (see Lemma 4.3) and $f_{z_{0}}^{\prime \prime}$ is a Lipschitzian function of $\left(D_{Z} Z\right)_{\mid z_{0}}, f_{z_{0}}^{\prime}, f_{z_{0}}$ and $z_{0}$, for the $C^{r}$ norms, the system (4.8) is of the form:

$$
\left.f_{z}^{\prime \prime}=\Phi\left(f_{z}^{\prime}, f_{z}, z\right) \text {, with } \Phi \text { Lipschitzian from } \mathrm{TG} \times \mathcal{G} \times I^{\prime} \text { to } C^{r}\left(\left(\frac{1}{2} I\right)^{n-1}, I^{n-1}\right)\right) .
$$

To obtain an O.D.E. in $\mathcal{G}$, we must check that $\Phi$ applies in $\mathrm{T} \mathcal{G}=\mathcal{F} \subset C^{r}\left(\left(\frac{1}{2} I\right)^{n-1}, I^{n-1}\right)$. We have seen that a curve $f=\left(f_{z}\right)_{z}$ in $C^{r}\left(\left(\frac{1}{2} I\right)^{n-1}, I^{n-1}\right)$ is a curve in $\mathcal{F}$ if and only if $f_{0} \in \mathcal{F}$ and for every $z, Z_{\mid z}$ satisfies (4.1). So it is a curve in $\mathcal{F}$ if and only if $f_{0} \in \mathcal{F}, f_{0}^{\prime} \in \mathcal{F}$ and the following derivative in $z$ of Relation (4.1) holds:

$$
D_{Z}\left(D_{\mathbf{X}} Z\right) \in \mathbf{X} \text { and } \forall s \leqslant k, D_{Z}\left(D_{\mathbf{Y}^{s}} Z\right) \in \mathbf{Y}^{s}
$$

Now, for each $s$ and any vector $Y^{s} \in \mathbf{Y}^{s}, D_{Z}\left(D_{\mathbf{Y}^{s}} Z\right)=R\left(Z, Y^{s}\right) Z+D_{Y^{s}}\left(D_{Z} Z\right)$, and similarly for $\mathbf{X}$, so (4.10) is equivalent to:

$$
\left\{\begin{array}{l}
\forall X \in \mathbf{X}, D_{X}\left(D_{Z} Z\right) \equiv R(X, Z) Z[\mathbf{X}] \\
\forall s \leqslant k, \forall Y^{s} \in \mathbf{Y}^{s}, D_{Y^{s}}\left(D_{Z} Z\right) \equiv R\left(Y^{s}, Z\right) Z\left[\mathbf{Y}^{s}\right]
\end{array} .\right.
$$

So, we are done if and only if, replacing $D_{Z} Z$ by $\alpha\left(f_{z}, f_{z}^{\prime}, z\right)^{\sharp}$, in (4.11), the relation is still satisfied. It is immediately the case as, by (4.2) in Lemma 4.3, for any $x \in \mathbf{X}$, $D_{X}\left(\alpha\left(f_{z}, f_{z}^{\prime}, z\right)^{\sharp}\right)=R(X, Z) Z$ and for any $s \leqslant k$ and $Y^{s} \in \mathbf{Y}^{s}, D_{Y^{s}}\left(\alpha\left(f_{z}, f_{z}^{\prime}, z\right)^{\sharp}\right)=$ $R\left(Y^{s}, Z\right) Z$.

So Relation (4.8) is an O.D.E. of order two in $\mathcal{F}$, with initial condition in $\mathcal{G} \times \mathrm{T} \mathcal{G}$ given by the second point of the lemma. The Cauchy-Lipschitz theorem gives the result.

Proof of Lemma 4.12. We have to prove " $\left(f_{z}\right)_{z}$ satisfies the conditions of Lemma $4.11 \Leftrightarrow$ it is as claimed by Lemma 4.12". Notice first that, if

$$
D_{Z} Z \equiv \alpha\left(f_{z_{0}}, f_{z_{0}}^{\prime}, z_{0}\right)^{\sharp}[\mathbf{X}],
$$

then for each two distributions $\mathbf{A}, \mathbf{B}$ among $\left\{\mathbf{X}, \mathbf{Y}^{s} ; 0 \leqslant s \leqslant k\right\}$ and for each coordinatevectors $A \in \mathbf{A}$ and $B \in \mathbf{B}$,

$$
\left(\mathbf{A} \perp \mathbf{B} \text { or } \mathbf{A}=\mathbf{B}=\mathbf{Y}^{0}\right) \Rightarrow g\left(D_{Z} D_{A} Z, B\right)=0 .
$$

Indeed, $g\left(D_{Z} D_{A} Z, B\right)=g(R(Z, A) Z, B)+g\left(D_{A} D_{Z} Z, B\right)$ and in turn $g\left(D_{A} D_{Z} Z, B\right)=$ $\alpha\left(f_{z_{0}}, f_{z_{0}}^{\prime}, z_{0}\right)(A, B)=g(R(A, Z) Z, B)$. In fact, (4.13), together with some limit conditions on $D_{Z} Z$ along the submanifolds $\left\{y^{s}=0\right\} \cap\left\{z=z_{0}\right\}$, is equivalent to (4.12). Lemma 4.12 is based essentially on this remark - the form $\alpha\left(f_{z_{0}}, f_{z_{0}}^{\prime}, z_{0}\right)$ was built in order to let Lemma 4.12 work. We detail the most part of the direct sense of the equivalence and leave the rest to the reader.

We prove that for each $z, \gamma_{z}$ is closed and that $g^{0}$ is everywhere the identity matrix.

Along $\mathcal{X}_{m}^{\perp}, \gamma_{0}$ vanish, so is closed. Let us show that $L_{Z}\left(\mathrm{~d} \gamma_{z}\right)=0$; let $A$ and $B$ be two 
coordinate-vectors among $\left(X,\left(\left(Y_{i}^{s}\right)_{i=1}^{n_{s}}\right)_{s=0}^{k}\right)$.

$$
\begin{aligned}
L_{Z}\left(\left(\mathrm{~d} \gamma_{z}\right)(A, B)\right) & =L_{Z}\left(L_{A} g(Z, B)-L_{B} g(Z, A)\right) \\
& =L_{Z}\left(g\left(D_{A} Z, B\right)-g\left(D_{B} Z, A\right)\right) \quad \text { as } D_{A} B=D_{B} A \\
& =g\left(D_{Z} D_{A} Z, B\right)-g\left(D_{Z} D_{B} Z, A\right) \quad \text { as } D_{A} Z=D_{Z} A \text { and } D_{B} Z=D_{Z} B \\
& =g(R(Z, A) Z, B)+g\left(D_{A} D_{Z} Z, B\right)-g(R(Z, B) Z, A)-g\left(D_{B} D_{Z} Z, A\right) \\
& =g\left(D_{A} D_{Z} Z, B\right)-g\left(D_{B} D_{Z} Z, A\right) \\
& =L_{A} g\left(D_{Z} Z, B\right)-L_{B} g\left(D_{Z} Z, A\right) \quad \text { again as } D_{A} B=D_{B} A \\
& =L_{A} \alpha\left(f_{z}, f_{z}^{\prime}, z\right)(B)-L_{B} \alpha\left(f_{z}, f_{z}^{\prime}, z\right)(A) \\
& =0 \quad \text { as } \alpha\left(f_{z}, f_{z}^{\prime}, z\right) \text { is closed. }
\end{aligned}
$$

So $L_{Z}\left(\mathrm{~d} \gamma_{z}\right)=0$, so for all $z, \gamma_{z}$ is closed. Similarly, on $\mathcal{X}_{m}^{\perp}, g_{z=0}^{0}$ is constant, equal to the identity matrix, as the coordinates on $\mathcal{X}_{m}^{\perp}$, given by Lemma 4.6, satisfy (4.5) and (4.6). We show finally that for all $z, \frac{\mathrm{d}}{\mathrm{d} z} g_{z}^{0}=0$. For each value of $z$, let us consider $\left(D_{Z}\right)_{\mid \mathbf{Y}^{0}}$ as a field of endomorphisms of $\mathbf{Y}^{0}$, defined along $\mathcal{X}_{z}^{\perp}$. On the one hand, at $z=0,\left(D_{Z}\right)_{\mid \mathbf{Y}^{0}}=0$ :

$$
g\left(D_{Z} Y_{i}^{0}, Y_{j}^{0}\right)=g\left(D_{Y_{i}^{0}} Z, Y_{j}^{0}\right)=L_{Y_{i}^{0}} g\left(Z, Y_{j}^{0}\right)-g\left(Z, D_{Y_{i}^{0}} Y_{j}^{0}\right)=0,
$$

as, by the second point of Lemma $4.11, Z \perp Y_{j}^{0}$ along $\mathcal{X}_{m}^{\perp}$, and by (4.5), $D_{Y_{i}^{0}} Y_{j}^{0}=0$ on $\mathcal{X}_{m}^{\perp}$. On the other hand, when the parameter $z$ runs, $\left(D_{Z}\right)_{\mid \mathbf{Y}^{0}}$ satisfies a homogeneous O.D.E. Indeed:

$$
\begin{aligned}
L_{Z} g\left(D_{Z} Y_{i}^{0}, Y_{j}^{0}\right) & =g\left(D_{Z} D_{Z} Y_{i}^{0}, Y_{j}^{0}\right)+g\left(D_{Z} Y_{i}^{0}, D_{Z} Y_{j}^{0}\right) \\
& =g\left(D_{Z} D_{Y_{i}^{0}} Z, Y_{j}^{0}\right)+g\left(D_{Z} Y_{i}^{0}, D_{Z} Y_{j}^{0}\right) \\
& =g\left(D_{Z} Y_{i}^{0}, D_{Z} Y_{j}^{0}\right) \quad \text { as, by }(4.13), g\left(D_{Z} D_{Y_{i}^{0}} Z, Y_{j}^{0}\right)=0
\end{aligned}
$$

Now $g\left(D_{Z} Y_{i}^{0}, D_{Z} Y_{j}^{0}\right)=g\left({ }^{t}\left(D_{Z}\right) D_{Z} Y_{i}^{0}, Y_{j}^{0}\right)$ where ${ }^{t}\left(D_{Z}\right)$ is the $g$-adjoint of $D_{Z}$. Hence, $L_{Z}\left(\left(D_{Z}\right)_{\mid \mathbf{Y}^{0}}\right)=\Phi\left(\left(D_{Z}\right)_{\mid \mathbf{Y}^{0}}, z\right)$ for $\Phi$ some bilinear continuous operator. So $\left.\left(D_{Z}\right)_{\mid \mathbf{Y}^{0}}\right)=0$ for all $z$; it follows quickly that $L_{z}\left(g_{z}^{0}\right)=0$ for all $z$. We are done.

4.13 Remark Notice that by construction, in adapted coordinates, $L_{Z} \gamma=\alpha\left(f_{z}, f_{z}^{\prime}, z\right)$. Indeed, take any coordinate-vector $Y \in \mathbf{X}^{\perp}, L_{Z} \gamma(Y)=L_{Z} g(Y, Z)=g\left(D_{Z} Y, Z\right)+g\left(Y, D_{Z} Z\right)$, now $g\left(D_{Z} Y, Z\right)=g\left(D_{Y} Z, Z\right)=\frac{1}{2} L_{Y} g(Z, Z)=0$ and, by $(4.8), g\left(Y, D_{Z} Z\right)=\alpha\left(f_{z}, f_{z}^{\prime}, z\right)(Y)$.

Proof of Proposition 3.9. Notice that for any coordinate-vector $Y_{i}^{0}, \check{Y}_{i}^{0}$ is parallel i.e. for all $V, D_{V} Y_{i}^{0} \in \mathbf{X}$. We take the notation of the proposition and set $\alpha=\alpha\left(f_{z}, f_{z}^{\prime}, z\right)$.

$$
\begin{aligned}
& \gamma(X) L_{Z}\left(\frac{1}{\gamma(X)} L_{A} \gamma(B)\right) \\
= & L_{Z} L_{A} \gamma(B)-\frac{1}{\gamma(X)} L_{Z} \gamma(X) L_{A} \gamma(B) \\
= & L_{A} \alpha(B)-\frac{1}{\gamma(X)} \alpha(X)\left(g\left(D_{A} Z, B\right)+g\left(Z, D_{A} B\right)\right) \quad \text { as } D_{Z} Z \equiv \alpha[\mathbf{X}] \\
= & \left(D_{A} \alpha\right)(B)-\alpha\left(D_{A} B\right)-\frac{1}{g(Z, X)} g\left(D_{Z} Z, X\right) g\left(Z, D_{A} B\right) \\
& \quad \text { as, if } \mathbf{A} \neq \mathbf{B}, D_{A} Z \in \mathbf{A} \perp \mathbf{B} \text { and, if } \mathbf{A}=\mathbf{B}=\mathbf{Y}^{0}, D_{A} Z=D_{Z} A \in \mathbf{X} \\
= & g(R(A, Z) Z, B)-g\left(D_{Z} Z, D_{A} B\right)-\frac{g\left(Z, D_{A} B\right)}{g(Z, X)} g\left(D_{Z} Z, X\right)
\end{aligned}
$$

Besides, if $\mathbf{A} \neq \mathbf{B}, D_{A} B=D_{B} A \in \mathbf{A} \cap \mathbf{B}=\mathbf{X}$ so in all cases $D_{A} B \in \mathbf{X}$, thus $D_{A} B=\frac{g\left(Z, D_{A} B\right)}{g(Z, X)} X$. Replacing in the last line above gives the result. 
Proof of Proposition 3.12. It is a long but not tricky calculation. First it is sufficient to prove the equality for $Y, Y^{\prime}, Y^{\prime \prime} \in \mathbf{Y}^{s}$ for each $s \in \llbracket 1, k \rrbracket$; else, the left side vanishes as $\check{g}$ is the product of the $\check{g}^{s}$ which satisfy the first point of (3.2) and the right side vanishes by Lemma 4.1. Then it suffices to prove it along $\check{\mathcal{Y}}_{\tilde{m}_{z}}^{s}=\left\{\check{y}^{r}=0 ; r \neq s\right\}$. Indeed, for any vector field $\check{Y}^{r}$ in $\check{\mathbf{Y}}^{r}$, with $r \neq s$ or $r=0, L_{\check{Y}^{r}}\left(\mathrm{~d}^{D}\left(\frac{\mathrm{d} \check{g}_{z}^{s}}{\mathrm{~d} z}\right)\left(\check{Y}, \check{Y}^{\prime}, \check{Y}^{\prime \prime}\right)\right)=0$ (straightforward) and:

$$
\begin{aligned}
L_{\check{Y}^{r}} g\left(R\left(\check{Y}, \check{Y}^{\prime}\right) \check{Y}^{\prime \prime}, Z\right)= & \left(D_{\check{Y}^{r}} g\right)\left(R\left(\check{Y}, \check{Y}^{\prime}\right) \check{Y}^{\prime \prime}, Z\right)+\Sigma \\
& \quad \text { where } \Sigma \text { is a sum of terms which vanish by Lemma 4.1, } \\
= & \left(D_{\check{Y}^{\prime}} g\right)\left(R\left(\check{Y}^{r}, \check{Y}^{\prime}\right) \check{Y}^{\prime \prime}, Z\right)+\left(D_{\check{Y}^{\prime}} g\right)\left(R\left(\check{Y}, \check{Y}^{r}\right) \check{Y}^{\prime \prime}, Z\right) \\
& \quad \text { by the second Bianchi identity } \\
= & \quad \text { again by Lemma } 4.1 \text { or, if } r=0, \text { directly, as } D_{\mathbf{Y}^{\circ}} g=0 .
\end{aligned}
$$

So let us take $\check{p} \in \check{\mathcal{Y}}_{\check{m}_{z}}^{s}$. To simplify the next calculations, we take a system $\left(\check{y}_{i}\right)_{i=1}^{n_{s}}$ of normal coordinates of $\check{\mathcal{Y}}_{\check{m}_{z}}^{s}$, centered at $\check{p}$, so at $\check{p}: \forall i, j, D_{\check{Y}_{i}} \check{Y}_{j}=0$. Setting $y_{i}=\check{y}_{i} \circ \pi$ and adding on $\mathcal{Y}_{m_{z}}^{s}$ the coordinate $x$ given by the adapted system of coordinates, we obtain a coordinate system $\left(x,\left(y_{i}\right)_{i=1}^{n_{s}}\right)$ of $\mathcal{Y}_{m_{z}}^{s}$ with the following properties: along $\mathcal{S}_{z}^{s}, \forall i, g\left(Y_{i}, Z\right)=0$ and at the point $p$ of $\mathcal{S}_{z}^{s}$ such that $\pi(p)=\check{p}, \forall i, j, D_{Y_{i}} Y_{j} \in \mathbf{X}_{p}$. Then we propagate those coordinates, from $p$, on $\cup_{z \in I} \mathcal{Y}_{m_{z}}^{s}$ by the flow of the coordinate vector field $Z=\frac{\partial}{\partial z}$ of the adapted coordinates. This flow preserves $\mathbf{Y}^{s}$, so for all $i, D_{Z} Y_{i}=D_{Y_{i}} Z \in \mathbf{Y}^{s}$.

It is sufficient to show (3.6) for $Y, Y^{\prime}, Y^{\prime \prime}$ among the $Y_{i}$ at $p$. Let $h$ be the bilinear form $\frac{\mathrm{d} \check{g}_{z}}{\mathrm{~d} z} ; D_{Y} h\left(Y^{\prime}, Y^{\prime \prime}\right)=g\left(D_{Y} D_{Z} Y^{\prime}, Y^{\prime \prime}\right)+g\left(D_{Y} D_{Z} Y^{\prime \prime}, Y^{\prime}\right)$. Indeed, $D_{Y} h\left(Y^{\prime}, Y^{\prime \prime}\right)=$ $L_{Y} h\left(Y^{\prime}, Y^{\prime \prime}\right)-h\left(D_{Y} Y^{\prime}, Y^{\prime \prime}\right)-h\left(Y^{\prime}, D_{Y} Y^{\prime \prime}\right)$ and:

$$
\begin{aligned}
& L_{Y} h\left(Y^{\prime}, Y^{\prime \prime}\right) \\
= & L_{Y} L_{Z} g\left(Y^{\prime}, Y^{\prime \prime}\right) \\
= & g\left(D_{Y} D_{Z} Y^{\prime}, Y^{\prime \prime}\right)+g(\underbrace{D_{Z} Y^{\prime}}_{\in \mathbf{Y}_{p}^{s}}, \underbrace{D_{Y} Y^{\prime \prime}}_{\in \mathbf{X}_{p}})+g(\underbrace{D_{Y} Y^{\prime}}_{\in \mathbf{X}_{p}}, \underbrace{D_{Z} Y^{\prime \prime}}_{\in \mathbf{Y}_{p}^{s}})+g\left(Y^{\prime}, D_{Y} D_{Z} Y^{\prime \prime}\right), \\
\bullet \quad & h\left(D_{Y} Y^{\prime}, Y^{\prime \prime}\right) \\
= & L_{Z} g\left(D_{Y} Y^{\prime}, Y^{\prime \prime}\right)-g\left(\left[Z, D_{Y} Y^{\prime}\right], Y^{\prime \prime}\right) \\
= & g\left(D_{Z} D_{Y} Y^{\prime}, Y^{\prime \prime}\right)+g(\underbrace{D_{Y} Y^{\prime}}_{\in \mathbf{X}_{p}}, \underbrace{D_{Z} Y^{\prime \prime}}_{\in \mathbf{Y}_{p}^{s}})-\left(g\left(D_{Z} D_{Y} Y^{\prime}, Y^{\prime \prime}\right)-g(\underbrace{D_{D_{Y} Y^{\prime} Z}}_{\propto D_{X} Z \in \mathbf{X}_{p}}, \underbrace{Y^{\prime \prime}}_{\mathbf{Y}_{p}^{s}})=0,\right.
\end{aligned}
$$

•symmetrically, $h\left(Y^{\prime}, D_{Y} Y^{\prime \prime}\right)=0$.

$$
\text { So: } \begin{aligned}
& \mathrm{d}^{D} h\left(Y, Y^{\prime}, Y^{\prime \prime}\right) \\
= & g\left(D_{Y} D_{Z} Y^{\prime}, Y^{\prime \prime}\right)+g\left(D_{Y} D_{Z} Y^{\prime \prime}, Y^{\prime}\right)-\left(g\left(D_{Y^{\prime}} D_{Z} Y, Y^{\prime \prime}\right)+g\left(D_{Y^{\prime}} D_{Z} Y^{\prime \prime}, Y\right)\right) \\
= & \left.g\left(R\left(Y, Y^{\prime}\right) Z, Y^{\prime \prime}\right)+g\left(D_{Y} D_{Y^{\prime \prime}} Z, Y^{\prime}\right)-g\left(D_{Y^{\prime}} D_{Y^{\prime \prime}} Z, Y\right)\right) \\
= & -g\left(R\left(Y, Y^{\prime}\right) Y^{\prime \prime}, Z\right)+g\left(R\left(Y, Y^{\prime \prime}\right) Z, Y^{\prime}\right)-g\left(D_{Y^{\prime \prime}} D_{Y} Z, Y^{\prime}\right) \\
& \quad-g\left(R\left(Y^{\prime}, Y^{\prime \prime}\right) Z, Y\right)+g\left(D_{Y^{\prime \prime}} D_{Y^{\prime}} Z, Y\right)
\end{aligned}
$$

By the first Bianchi identity, $g\left(R\left(Y, Y^{\prime \prime}\right) Z, Y^{\prime}\right)-g\left(R\left(Y^{\prime}, Y^{\prime \prime}\right) Z, Y\right)=-g\left(R\left(Y, Y^{\prime}\right) Y^{\prime \prime}, Z\right)$. Finally as, along $\mathcal{S}_{z}^{s}$, for all $i, g\left(Y_{i}, Z\right)=0$, one checks that: $g\left(D_{Y^{\prime \prime}} D_{Z} Y, Y^{\prime}\right)=L_{Y^{\prime \prime}} g\left(D_{Z} Y, Y^{\prime}\right)$ $=\frac{1}{2} L_{Y^{\prime \prime}} L_{Z} g\left(Y, Y^{\prime}\right)$, so $-g\left(D_{Y^{\prime \prime}} D_{Y} Z, Y^{\prime}\right)+g\left(D_{Y^{\prime \prime}} D_{Y^{\prime}} Z, Y\right)=\frac{1}{2} L_{Y^{\prime \prime}} L_{Z}\left(-g\left(Y, Y^{\prime}\right)+g\left(Y^{\prime}, Y\right)\right)$ $=0$. We are done. 
4.14 Remark So, adapted coordinates appear as solutions of an O.D.E. in an infinite dimensional Banach space. This O.D.E. cannot be "factored", to be turned into an O.D.E. on $I^{n-1}$ itself, unless the holonomy group acts trivially on $\mathbf{X}$. In this particular case, the problem becomes a lot easier to solve, see [Bo00]. Notably, if $n_{0}=0$ and $k=1$ (only "one block" $\check{\mathbf{Y}}^{1}$ ) the O.D.E. on the field $Z$ is exactly the equation of the geodesics: along $\mathcal{X}_{m}^{\perp}, Z \perp \operatorname{span}\left(Y_{i}\right)_{i=1}^{n_{1}}, g(Z, Z)=0$ and $g(Z, X)=1$; then everywhere, $D_{Z} Z=0$ gives the adapted coordinates in this case, with moreover $\gamma \equiv 0$. The reader can check it immediately. So all the difficulty, in Theorem 3.7, comes from the case where $H^{0}$ acts non trivially on $\mathbf{X}$.

\section{The metrics giving each type of holonomy representation}

\section{5. a A more detailed description of the algebras "of type 3 and 4"}

First, if a holonomy algebra $\mathfrak{h}$ is of type 3 or 4 , as defined in Theorem 2.1, the nature of the Lie algebra $\mathfrak{r}$ and of the map $\psi$ appearing in that theorem follows quickly from Proposition 2.5; we give it in Lemma 5.1 below, left to the reader. Based on the notation of Proposition 2.5, we denote by $\check{\mathfrak{h}}^{s}$ the Lie algebra of $\check{H}^{s}$.

Here we call reductive a Lie algebra $\mathfrak{r}$ which is the direct sum of two ideals $\mathfrak{r}=\mathfrak{s} \oplus \mathfrak{t}$ with $\mathfrak{s}$ semi-simple and $\mathfrak{t}$ abelian, be the subgroup $\exp (\mathfrak{t})$ closed or not in $\exp (\mathfrak{r})$. In that sense, all subalgebra of $\mathfrak{s o}_{n}(\mathbb{R})$ is reductive, as $\mathrm{SO}_{n}(\mathbb{R})$ is compact.

5.1 Lemma Let $\mathfrak{h}_{m}$ be a holonomy algebra of type 3 or 4 and $\mathfrak{h}_{m}=\mathfrak{s}+\mathfrak{t}$ be its decomposition into its semi-simple and abelian ideals. As $\mathrm{SO}_{n}(\mathbb{R})$ is compact, every Lie subalgebra of $\mathfrak{s o}_{n}(\mathbb{R})$ admits indeed such a decomposition (the subgroup $\exp (\mathfrak{t})$ being not necessarily closed in $\exp (\mathfrak{r})$ ). For each $s$,

- either $\check{\mathfrak{h}}_{\check{m}}^{s} \subset \mathfrak{s}$

- or $\check{\mathfrak{h}}_{\check{m}}^{s}=\check{\mathfrak{t}}_{\check{m}}^{s} \oplus \check{\mathfrak{s}}_{\check{m}}^{s}$, where $\check{\mathfrak{t}}_{\check{m}}^{s}=\mathfrak{t} \cap \check{\mathfrak{h}}_{\check{m}}^{s}$ and $\check{\mathfrak{s}}_{\breve{m}}^{s}=\mathfrak{s} \cap \check{\mathfrak{h}}_{m}^{s}$ are commuting ideals.

In the second case, $\operatorname{dim} \check{\mathbf{Y}}_{\check{m}}^{s}$ is even and there exists a complex structure $J_{\check{m}}^{s} \in \mathrm{SO}\left(\check{\mathbf{Y}}_{\check{m}}^{s}, \check{g}_{\check{m}}^{s}\right)$ i.e. $\left(J_{m}^{s}\right)^{2}=-\mathrm{Id}$, such that $\check{\mathfrak{s}}_{m}^{s} \subset \mathfrak{s u}\left(\check{\mathbf{Y}}_{\check{m}}^{s}, \check{g}_{\check{m}}^{s}, J_{\check{m}}^{s}\right)$ and that $\check{\mathfrak{t}}_{\grave{m}}^{s}=\operatorname{span}\left(J_{\dddot{m}}^{s}\right) \simeq \mathbb{R}$ is the center of $\mathfrak{u}\left(\check{\mathbf{Y}}_{\check{m}}^{s}, \check{g}_{\check{m}}^{s}, J_{\check{m}}^{s}\right)$.

5.2 Consequence/Notation Reordering possibly the indices $s$, we suppose that the $\check{\mathfrak{h}}_{\dddot{m}}^{s}$ are in the second case if and only if $s \in \llbracket 1, k^{\prime} \rrbracket$. Besides, for each $s \leqslant k^{\prime}$, the map $t \mapsto t J_{m}^{s}$ provides a canonical isomorphism from $\mathbb{R}$ to $\check{\mathfrak{t}}_{\tilde{m}}^{s}$. By this means, $\mathfrak{t}=\oplus_{s=1}^{k^{\prime}} \check{\mathfrak{t}}_{\breve{m}}^{s}$ is canonically identified with $\mathbb{R}^{k^{\prime}}$. Therefore, if $\mathfrak{h}_{m}$ is of type 3 , the map $\psi$ is of the form:

$$
\begin{array}{cc}
\mathfrak{t} \simeq \mathbb{R}^{k^{\prime}} & \rightarrow \mathbb{R} \\
\psi: \quad\left(t_{1}, \ldots, t_{k^{\prime}}\right) & \mapsto \sum_{i=1}^{k^{\prime}} t_{s} \lambda^{s}
\end{array} \text { for a certain } \lambda(\psi)=\left(\lambda^{s}\right)_{s=1}^{k^{\prime}} \in \mathbb{R}^{k^{\prime}} \quad\left(k^{\prime} \leqslant k\right)
$$

If $\mathfrak{h}_{m}$ is of type 4 , let us denote by $\mathbf{Y}_{m}^{0 \prime}$ the binded subspace of $\mathrm{T}_{m} \mathcal{M}$, according to Terminology 2.2 (notice that $\mathbf{Y}_{m}^{0 \prime} \subset \mathbf{Y}_{m}^{0}$ ), and as usual $\check{\mathbf{Y}}_{m}^{0 \prime}=\mathbf{Y}_{m}^{0 \prime} / \mathbf{X}_{m}$. With that notation, independent of the chosen coordinates, $\psi$ maps $T$ onto $\check{\mathbf{Y}}_{m}^{0 \prime *} \otimes \mathbf{X}_{m}$. Once (arbitrarily) chosen a nonzero vector $X \in \mathbf{X}_{m}, \psi$ can be seen with values in $\tilde{\mathbf{Y}}_{\dot{m}}^{0 / *}$. So, If $\mathfrak{h}_{m}$ is of type $4, \psi$ is of the form:

$$
\begin{array}{ccc}
\mathfrak{t} \simeq \mathbb{R}^{k^{\prime}} & \rightarrow & \mathbb{R} \\
\psi: \quad\left(t_{1}, \ldots, t_{k^{\prime}}\right) & \mapsto \sum_{i=1}^{k^{\prime}} t_{s} \Lambda^{s}
\end{array} \text { for a certain } \Lambda(\psi)=\left(\Lambda^{s}\right)_{s=1}^{k^{\prime}} \in\left(\check{\mathbf{Y}}_{\check{m}}^{0 / *}\right)^{k^{\prime}} \quad\left(k^{\prime} \leqslant k\right)
$$


Eventually, reordering again the indices $s$ and decreasing possibly $k^{\prime}$ to let apart the null $\lambda_{s}$ or $\Lambda_{s}$, we can suppose that none of them vanishes. Notice that, as $\psi$ is onto, the $\left(\lambda^{s}\right)_{s=1}^{k^{\prime}}$ and $\left(\Lambda^{s}\right)_{s=1}^{k^{\prime}}$ span, respectively, $\mathbb{R}$-so $k^{\prime} \geqslant 1-$ and $\check{\mathbf{Y}}_{\check{m}}^{0 / *}$-so $k^{\prime} \geqslant \operatorname{dim} \check{\mathbf{Y}}_{\check{m}}^{0 / *}$.

5.3 Remark Despite their similar appearence, representations of type 3 and 4 are quite different. Indeed, with a representation of type 3 is associated the family of scalars $\left(\lambda^{s}\right)_{s=1}^{k^{\prime}}$. This corresponds, by exponentiation in the holonomy group, to a family of angles: an element $e \in \exp (\mathfrak{h})$, acting trivially on all $\check{\mathbf{Y}}^{s}$ except $\check{\mathbf{Y}}^{r}$ for some $r \in \llbracket 1, k^{\prime} \rrbracket$, magnifies $\mathbf{X}_{m}$ by 2 if and only if it "rotates" the space $\left(\check{\mathbf{Y}}_{m}^{r}, J_{m}^{r}\right)$, of the angle $\frac{\pi}{2} \frac{\ln 2}{\lambda^{r}}$ i.e. if $e=\exp (h)$ with $h$ of complex trace $\operatorname{tr}_{J^{r}}\left(h_{\mid \check{\mathbf{Y}}_{m}^{r}}\right)=\frac{\ln 2}{\lambda^{r}}$. Nothing similar appears for type 4.

\section{5. b Some auxiliary definitions}

To state Theorem 5.14, we need some auxiliary concepts. If $\mathcal{U}$ is an open subset of $\mathbb{R}^{N}, N$ even, we view here a Kähler metric on $\mathcal{U}$ as a pair $(J, g)$ with $J$ a complex structure on $\mathcal{U}$ i.e. an integrable field of endomorphisms such that $J^{2}=-$ Id and $g$ a Riemannian metric on $\mathcal{U}$ such that $J$ is $g$-orthogonal and parallel for the Levi-Civita connection of $g$.

5.4 Definition A one-parameter family $\left(J_{t}, g_{t}\right)_{t \in I}$ of Kähler metrics on $\mathcal{U}$ is called admissible if, denoting by $D_{t}$ the Levi-Civita connection of $g_{t}$ :

- The field of endomorphisms $\frac{\mathrm{d}}{\mathrm{d} t} J_{t}$ is $g_{t}$-selfadjoint,

- The 1-form $U \mapsto \operatorname{tr}_{g_{t}}\left[\mathrm{~d}^{D_{t}}\left(\frac{\mathrm{d} g}{\mathrm{~d} t}\right)_{t}\left(\cdot, J_{t} \cdot, U\right)\right]$ do not everywhere vanish on $\mathcal{U}$.

5.5 Remark Any family $\left(J_{t}, g_{t}\right)_{t \in I}$ of Kähler metrics can be written $\left(\left(\varphi_{t}\right)_{*} J_{0},\left(\varphi_{t}^{-1}\right)^{*} \underline{g}_{t}\right)_{t \in I}$, where $\left(\varphi_{t}\right)_{t \in I}$ is a family of diffeomorphisms mapping $J_{0}$-complex coordinates on $J_{t}$-complex coordinates: $\left(\varphi_{t}\right)_{*} J_{o}=J_{t}$, and where $\left(g_{t}\right)_{t \in I}=\left(\left(\varphi_{t}\right)^{*} g_{t}\right)_{t \in I}$ is a family of $J_{0}$-Kähler metrics. So the set of families $\left(J_{t}, g_{t}\right)_{t \in I}$ of Kähler metrics is parametrized by the set of families $\left(g_{t}, \varphi_{t}\right)_{t \in I}$, with $\left(\varphi_{t}\right)_{t \in I}$ a family of diffeomorphisms from $\mathcal{U}$ to $\varphi_{t}(\mathcal{U})$ and $\left(g_{t}\right)_{t \in I}$ a family of $\bar{J}_{0}$-Kähler metrics on $\mathcal{U}$. In turn, $\left(\varphi_{t}\right)_{t \in I}$ can be seen as the (non autonomous) flow of some vector field $\left(V_{t}\right)_{t \in I}$ on $\varphi_{t}(\mathcal{U}): \frac{\mathrm{d}}{\mathrm{d} t} \varphi_{t}=V_{t} \circ \varphi_{t}$. The datum of $\left(\varphi_{t}\right)_{t \in I}$ is equivalent to that of $\left(V_{t}\right)_{t \in I}$ or to that of $\left(W_{t}\right)_{t \in I}=\frac{\mathrm{d}}{\mathrm{d} t} \varphi_{t}=\left(\mathrm{d} \varphi_{t}^{-1} \cdot V_{t}\right)_{t \in I}$, family of vector fields on $\mathcal{U}$.

5.6 Proposition We use here the notation introduced in Remark 5.5.

(a) The set $\mathcal{K}$ of the families $\left(J_{t}, g_{t}\right)_{t \in I}$ of Kähler metrics on $\mathcal{U}$ satisfying (5.3) is parametrized by the datum of $J_{0}$ and by the families $\left(\underline{g}_{t}, W_{t}\right)_{t \in I}$ where:

- $\left(\underline{g}_{t}\right)_{t \in I}$ is a family of $J_{0}$-Kähler metrics,

- $\left(W_{t}\right)_{t \in I}=\left(\left(\partial f_{t}+\overline{\partial f_{t}}\right)^{\sharp t}\right)_{t \in I}$ where $\left(f_{t}\right)_{t \in I}$ is any family of functions $f_{t} \in C^{\infty}(\mathcal{U}, \mathbb{C}), \partial$ is the $J_{0}$-complex derivative and $\sharp_{\underline{t}}^{t}$ is the musical isomorphism associated with $\underline{g}_{t}$.

In particular, such families exist; besides, for each $t, g_{t}$ is Ricci-flat if and only if $\underline{g}_{t}$ is.

(b) On $\mathcal{K},(5.4)$ is the negation of an algebraic condition on the 2-jet of each $\left(J_{t}, g_{t}\right)$, which is satisfied by at least one element of $\mathcal{K}$, moreover Ricci-flat for all $t$. So "almost all" (Ricci-flat or not) elements of $\mathcal{K}$ satisfy (5.4) i.e. are adapted. More precisely, these elements form a residual set in the Kähler Ricci-flat metrics, for example in the $C^{2}$ topology.

5.7 Remark In other words, the latter point of (a) in Proposition 5.6 means that $W_{t}$ is the sum of a gradient and of a symplectic gradient, with respect to the $J_{0}$-Kähler symplectic form. This always holds in real dimension 2, but is a strong condition in greater dimension. 
5.8 Remark With the notation of Remark 5.5, the set of the families $\left(J_{t}, g_{t}\right)_{t \in I}$ of Kähler metrics, with $J_{0}$ fixed, is, more precisely, in bijection with $\mathcal{E} / \sim$, where $\mathcal{E}$ is the set of the families $\left(\underline{g}_{t}, \varphi_{t}\right)_{t \in I}$ as in 5.5, and $\left(\underline{g}_{t}, \varphi_{t}\right) \sim\left(\underline{g}_{t}^{\prime}, \varphi_{t}^{\prime}\right)$ if $\varphi_{t}^{\prime} \circ \varphi_{t}^{-1}$ is $J_{0}$-holomorphic and and $\underline{g}_{t}^{\prime}=\left(\varphi_{t}^{\prime} \circ \varphi_{t}^{-1}\right)^{*} \underline{g}_{t}$. So, in $5.5,\left(\varphi_{t}\right)_{t \in I}$ can be seen as "defined up to right composition with a $J_{0}$-biholomorphism".

5.9 Definition We now consider again a metric $g$ on $\mathcal{M} \simeq I^{n}$, written in adapted coordinates. We suppose that, for some $k^{\prime} \in \llbracket 1, k \rrbracket$, the metrics $\left(\check{g}_{z}^{s}\right)_{s=1}^{k^{\prime}}$ are Kähler with respect to some complex structure $J_{z}^{s}$.

Take $\lambda=\left(\lambda_{s}\right)_{s=1}^{k^{\prime}} \in \mathbb{R}^{k^{\prime}}$; a function $f$ on $\mathcal{M}$ is called $\left(\left(\check{g}_{z}\right)_{z \in I}, \lambda\right)$-binded if:

- $f$ only depends on the coordinates $\left(y^{s}\right)_{s=1}^{k^{\prime}}$ and $z$,

- $\forall s \in \llbracket 1, k^{\prime} \rrbracket, f\left(L_{Y_{i}^{s}} L_{Z} \ln f\right)_{i=1}^{n_{s}}=\lambda^{s}\left(\operatorname{tr}_{\check{g}_{z}^{s}}\left(\mathrm{~d}^{\check{D}_{z}^{s}}\left(\frac{\mathrm{d}}{\mathrm{d} z} \check{g}_{z}^{s}\right)\left(\cdot, J_{z}^{s} \cdot \check{Y}_{i}^{s}\right)\right)\right)_{i=1}^{n_{s}}$.

In case $n_{0}>0$, let $\check{\mathbf{Y}}_{\check{m}}^{0 \prime}$ be a subspace of $\check{\mathbf{Y}}_{\check{m}}^{0}$ and $\Lambda=\left(\Lambda^{s}\right)_{s=0}^{k^{\prime}} \in\left(\check{\mathbf{Y}}_{\check{m}}^{0 / *}\right)^{k^{\prime}}$ a $k^{\prime}$-tuple of vectors of the dual space of $\check{\mathbf{Y}}_{\check{m}}^{0 \prime}$, with $k^{\prime} \leqslant k$. As the holonomy group acts trivially on $\check{\mathbf{Y}}_{\check{m}}^{0}$, $\check{\mathbf{Y}}_{\check{m}}^{0 \prime}$ gives rise, by parallel transport, to a parallel distribution $\check{\mathbf{Y}}^{0 \prime}$. If $\check{\alpha}$ is a one-form with variable in $\check{\mathbf{Y}}^{0 \prime}$, defined along $\check{\mathcal{M}}$, i.e. a section of $\check{\mathbf{Y}}^{0 / *} \check{\mathcal{M}}$, $\check{\alpha}$ is called $\left(\left(\check{g}_{z}\right)_{z \in I}, \Lambda\right)$-binded if:

- $\check{\alpha}$ only depends on the coordinates $\left(y^{s}\right)_{s=1}^{k^{\prime}}$ and $z$,

- $\forall s \in \llbracket 1, k^{\prime} \rrbracket,\left(L_{\check{Y}_{i}^{s}} L_{\check{Z}} \check{\alpha}\right)_{i=1}^{n_{s}}=\left(\operatorname{tr}_{\check{g}_{z}^{s}}\left(\mathrm{~d}^{\check{D}_{z}^{s}}\left(\frac{\mathrm{d}}{\mathrm{d} z} \check{g}_{z}^{s}\right)\left(\cdot, J_{z}^{s} \cdot, \check{Y}_{i}^{s}\right)\right) \Lambda^{s}\right)_{i=1}^{n_{s}}$.

5.10 Remarks • The terms $L_{\check{Y}_{i}^{s}} L_{\check{Z}} \check{\alpha}_{\mid \check{\mathbf{Y}}^{0 \prime}}$, apparently very dependent on the coordinates in which they are expressed, are a second derivative of $\check{\alpha}$ in an intrinsic sense. Indeed, in adapted coordinates, the coordinate-vector fields $\left(Y_{i}^{0}\right)_{i=1}^{n_{0}}$ are parallel — which is possible as

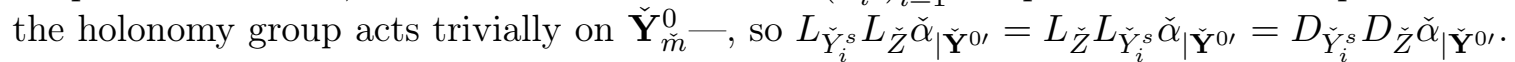

- Proposition 7.7 makes more explicit, in some cases, the terms $\operatorname{tr}_{\check{g}_{z}^{s}}(\ldots)$.

Eventually, the following lemma gives the link between "admissible" families of metrics and our topic: Lorentzian holonomy algebras.

5.11 Lemma For any $s \in \llbracket 1, k \rrbracket$, there is a complex structure $J^{s} \in \mathrm{SO}\left(\check{\mathbf{Y}}_{\check{m}}^{s}, g_{\check{m}}^{s}\right)$ such that

$$
\check{\mathfrak{h}}_{\check{m}}^{s} \subset \mathfrak{u}\left(\check{\mathbf{Y}}_{\check{m}}^{s}, g_{\check{m}}^{s}, J_{\check{m}}^{s}\right)
$$

if and only if there exists a one-parameter family $J_{z}^{s}$ of complex structures of $\check{\mathcal{Y}}_{\tilde{m}_{z}}^{s}$, such that $J_{0}^{s}=J^{s}$ and that $\left(g_{z}^{s}, J_{z}^{s}\right)_{z \in I}$ satifies Condition (5.3) of definition 5.4. If moreover, for each $z, g_{z}^{s}$ is Ricci-flat, then

$$
\check{\mathfrak{h}}_{\check{m}}^{s} \not \subset \mathfrak{s u}\left(\check{\mathbf{Y}}_{\check{m}}^{s}, g_{\check{m}}^{s}, J_{\check{m}}^{s}\right)
$$

if and only if $\left(g_{z}^{s}, J_{z}^{s}\right)_{z \in I}$ is admissible, i.e. satisfies also Condition (5.4) of Definition 5.4.

\section{5. c The germs of metrics, classified according to their holonomy repre- sentation}

5.12 Notation In this section, $g$ is a reducible-indecomposable Lorentzian metric given by $\left(\left(\left(g_{z}^{s}\right)_{z \in I}\right)_{s=0}^{k},\left(\gamma_{z}\right)_{z \in I}\right)$ in adapted coordinates applying in $I^{n}$, identified with the manifold.

We determine which $g_{z}^{s}$ and $\gamma_{z}$ let the holonomy algebra $\mathfrak{h}$ of $g$ be of type $1,2,3$ or 4 . First, the Ambrose-Singer theorem gives a translation of Theorem 2.1 in terms of relations, at every point, between some components of the curvature tensor. 
5.13 Proposition The holonomy algebra $\mathfrak{h}$ of $g$ is of type 3 , with $k^{\prime}$-tuple $\lambda(\psi)=\left(\lambda^{s}\right)_{s=1}^{k^{\prime}} \in$ $\mathbb{R}^{k^{\prime}}$ if and only if:

- for every $s \in \llbracket 1, k^{\prime} \rrbracket$ and every $z$, there is a complex structure $J_{z}^{s} \in \mathrm{SO}\left(\check{\mathbf{Y}}^{s}, \check{g}^{s}\right)$, such that the field $J_{z}^{s}$ (denoted also by $J^{s}$ ) is parallel on $\mathcal{M}$;

besides for every $z, \check{g}_{z}^{s}$ is $J_{z}^{s}$-Kähler, Ricci-flat,

- at any $p:\left\{\begin{array}{l}s=0 \text { or } s>k^{\prime} \Rightarrow g\left(R\left(\mathbf{Y}_{p}^{s}, Z\right) X, Z\right)=\{0\} \\ 1 \leqslant s \leqslant k^{\prime} \Rightarrow \forall Y \in \mathbf{Y}_{p}^{s}, g(R(Y, Z) X, Z)=\left(\operatorname{tr}_{J^{s}}\left(R(Y, Z)_{\mid \check{\mathbf{Y}}^{s}}\right)\right) \lambda^{s}\end{array}\right.$

- for any $s \in \llbracket 1, k^{\prime} \rrbracket$, at some point $q: \exists Y \in \mathbf{Y}_{q}^{s}: \operatorname{tr}_{J^{s}}\left(R(Y, Z)_{\mid \check{\mathbf{Y}}^{s}}\right) \neq 0$,

with $\operatorname{tr}_{J^{s}}$ standing for the $J^{s}$-complex trace of the $J^{s}$-complex endomorphisms $R(*, *)_{\mid \check{\mathbf{Y}}^{s}}$.

The algebra $\mathfrak{h}$ is of type 4 , with $k^{\prime}$-tuple $\Lambda(\psi)=\left(\Lambda^{s}\right)_{s=1}^{k^{\prime}} \in\left(\check{\mathbf{Y}}^{0 / *}\right)^{k^{\prime}}$ if and only if the holonomy group acts trivially on $\mathbf{X}_{m},(5.7)$, (5.8) and (5.10) hold and, instead of (5.9):

$$
\text { - at any } p:\left\{\begin{array}{l}
s=0 \text { or } s>k^{\prime} \Rightarrow g\left(R\left(\mathbf{Y}_{p}^{s}, Z\right) \mathbf{Y}_{p}^{0 \prime}, Z\right)=\{0\} \\
1 \leqslant s \leqslant k^{\prime} \Rightarrow \forall Y \in \mathbf{Y}_{p}^{s}, g\left(R(Y, Z)_{\mid \check{\mathbf{Y}}_{p}^{0 \prime}} \cdot Z\right)=\left(\operatorname{tr}_{J^{s}}\left(R(Y, Z)_{\mid \check{\mathbf{Y}}^{s}}\right)\right) \Lambda^{s}
\end{array}\right.
$$

Now here is the theorem itself. The terms "admissible" and " $\left(\left(\check{g}_{z}\right)_{z}, \lambda\right)$-binded" are defined in the next paragraph.

5.14 Theorem With the notation of this section and of Theorem 2.1:

(a) $\mathfrak{h}$ is of type 2 or 4 if and only if $(\gamma-\mathrm{d} x)$ is the pull back $\pi^{*} \check{\gamma}$ by $\pi$ of a 1 -form $\check{\gamma}$ of $\check{\mathcal{M}}$. It is of type 4 , with binded subspace $\mathbf{Y}_{m}^{0 \prime}$ and with $k^{\prime}$-tuple $\Lambda(\psi)=\left(\Lambda^{s}\right)_{s=1}^{k^{\prime}} \in\left(\check{\mathbf{Y}}^{0 / *}\right)^{k^{\prime}}$ (see Formula (5.2)) if and only if, additionally:

- for $1 \leqslant s \leqslant k^{\prime}$, each $\left(g_{z}^{s}\right)_{z \in I}$ is an admissible family of $J_{z}^{s}$-Kähler, Ricci-flat Riemannian metrics,

- $\check{\gamma}_{\mid \mathbf{Y}^{0}}$ is $\left(\left(\check{g}_{z}\right)_{z \in I}, \Lambda(\psi)\right)$-binded, this condition being satisfied, for a given $\left(\left(\check{g}_{z}\right)_{z \in I}, \Lambda(\psi)\right)$, by a unique form $\check{\gamma}_{\mid \mathbf{Y}^{0 \prime}}$.

(b) Else, $\mathfrak{h}$ is of type 1 or 3. It is of type 3, with $k^{\prime}$-tuple $\lambda(\psi)=\left(\lambda^{s}\right)_{s=1}^{k^{\prime}} \in\left(\mathbb{R}^{*}\right)^{k^{\prime}}$ (see Formula (5.1)) if and only if, additionally:

- for $1 \leqslant s \leqslant k^{\prime}$, each $\left(g_{z}^{s}\right)_{z \in I}$ is an admissible family of $J_{z}^{s}$-Kähler, Ricci-flat Riemannian metrics,

- $\gamma(X)$ is $\left(\left(\check{g}_{z}\right)_{z \in I}, \lambda(\psi)\right)$-binded, this condition being satisfied, for a given $\left(\left(\check{g}_{z}\right)_{z \in I}, \lambda(\psi)\right)$, by a unique function $\gamma(X)$.

5.15 Corollary Theorem 5.14 parametrizes, up to the action of a subgroup of $\mathrm{GL}(n-1, \mathbb{R})$, the set of germs of metrics of the "exceptional" types 3 and 4.

Indeed, in adapted coordinates and if you choose moreover, for each $s \geqslant 1$, the coordinate-vectors $\left(Y_{i}^{s}\right)_{i=1}^{n_{s}}$, in the hypersurface $\mathcal{X}_{0}^{\perp}$, equal to the exponential of linear orthonormal coordinate-vectors on $\mathrm{T}_{m} \mathcal{Y}_{0}^{s}$ (equivalently, if you choose each submanifold $\mathcal{S}_{0}^{s}=\{x=0, z=$ $0, y^{r}=0$ for $\left.r \neq s\right\}$ such that $\mathcal{S}_{0}^{s}=\exp \left(\mathrm{T}_{m} \mathcal{S}_{0}^{s}\right)$, and each set of coordinates $\left(\check{y}_{i}^{s}\right)_{i=1}^{n_{s}}$ of $\check{\mathcal{Y}}_{0}^{s}$ as normal coordinates centered at $\check{m})$, then:

(a) A metric of type 3 (respectively 4), with prescribed $k^{\prime}$-tuple $\lambda(\psi)$, (respectively with prescribed $k^{\prime}$-tuple $\left.\lambda(\psi)\right)$ is given by the independent and arbitrary data of: 
- a nonzero vector $X$ of $\mathbf{X}_{m}=\mathrm{T}_{m} \mathcal{X}$,

- a $k^{\prime}$-tuple of 1-parameter families $\left(\left(g_{z}^{s}\right)_{z \in]-\varepsilon, \varepsilon[}\right)_{s=1}^{k^{\prime}}$ of admissible, Ricci-flat Kähler Riemannian metrics, such that, at $z=0$, each $g_{0}^{s}$ is given in normal coordinates centered at zero and that, if $\vec{r}$ is the normal radius vector in $\mathbb{R}^{n_{s}},\left.\frac{\mathrm{d}}{\mathrm{d} z}\right|_{z=0} g_{z}^{s}(\vec{r}, \vec{r})=0$,

- a $k$-tuple of 1-parameter families $\left(\left(g_{z}^{s}\right)_{z \in]-\varepsilon, \varepsilon[}\right)_{s=k^{\prime}+1}^{k}$ of Riemannian metrics, satisfying at $z=0$ the same condition as the $\left(g_{z}^{s}\right)_{s=1}^{k^{\prime}}$ just above,

- [for a metric of type 3] the value of the closed form $\gamma$ on each submanifold $\{x=$ $\left.0, z=z_{0}\right\}$ i.e. the value of the $\gamma\left(Y_{i}^{s}\right)$ along this manifold (with the constraints given by Theorem 3.7 i.e. for each $s$ and $i \leqslant n_{s}, \gamma\left(Y_{i}^{s}\right)=0$ along each $\mathcal{S}_{z}^{s}$ and $\gamma=0$ on $\left.\mathcal{X}_{0}^{\perp}=\{z=0\}\right)$.

- [respectively, for a metric of type 4] the value of the closed form $\check{\gamma}$ on each hypersurface $\left\{z=z_{0}\right\}$ of the quotient $\check{\mathcal{M}}=\mathcal{M} / \mathcal{X}$ i.e. the value of the $\check{\gamma}\left(\check{Y}_{i}^{s}\right)$ along this hypersurface (with the constraints given by Theorem 3.7 i.e. for each $s$ and $i \leqslant n_{s}, \gamma\left(Y_{i}^{s}\right)=0$ along each $\mathcal{Y}_{z}^{s}$ and $\gamma=0$ on $\left.\check{\mathcal{X}}_{0}^{\perp}=\{z=0\}\right)$.

(b) If $X$ is replaced by $\mu X, \mu \in \mathbb{R}^{*}$, to obtain the same germ of metric, one must replace the $\left(\left(g_{z}^{s}\right)_{z \in]-\varepsilon, \varepsilon[}\right)_{s=1}^{k}$ by $\left(g_{\frac{1}{\mu} z}\right)_{z \in]-\mu \varepsilon, \mu \varepsilon[}$ and $\gamma_{\mid\left(x, y^{0}, \ldots, y^{k}, z\right)}$ by $\frac{1}{\mu} \gamma_{\mid\left(\mu x, y^{0}, \ldots, y^{k},(1 / \mu) z\right)}$.

(c) For a given choice of $X$, two data as described in (a) give isometric germs if and only if the $\left(\left(g_{z}^{s}\right)_{z \in]-\varepsilon, \varepsilon[}\right)_{s=1}^{k}$ and $\gamma$ are on the same orbit of an action of $\prod_{s=1}^{k} \mathrm{SO}\left(n_{s}, \mathbb{R}\right) \times \mathbb{R}^{n_{s}}$.

Proof. We do it for the case of a metric of type 3. Type 4 is similar. In adapted coordinates, a germ of metric is given by the independent and arbitrary data of the 1-form $\gamma$ and of the quotient families of metrics $\left(\left(g_{z}^{s}\right)_{z \in]-\varepsilon, \varepsilon[}\right)_{s=1}^{k}$. Prescribing moreover that the coordinate-vectors $Y_{i}^{s}$ are the exponential of linear coordinate-vectors of $\mathrm{T}_{m} \mathcal{X}_{0}^{\perp}$ amounts to require that, for each $s$ :

- $g_{0}^{s}$ is given in normal coordinates centered at zero,

- $\left.\frac{\mathrm{d}}{\mathrm{d} z}\right|_{z=0} g_{z}^{s}(\vec{r}, \vec{r})=0$, with $\vec{r}$ is the normal radius vector in $\mathbb{R}^{n_{s}}$.

The first point is immediate, as the $\left(\check{y}_{i}^{s}\right)_{i=1}^{n_{s}}$ are normal coordinates of $\check{\mathcal{Y}}_{m}^{s}$ if and only if $g_{0}^{S}$ is given in normal coordinates centered at zero. For the second point, notice that:

$$
\begin{aligned}
& \mathcal{S}_{m}^{s}=\exp \left(\mathrm{T}_{m} \mathcal{S}_{m}^{s}\right) \\
\Leftrightarrow & g\left(D_{\vec{r}} \vec{r}, Z\right)=0 \text { along } \mathcal{Y}_{m}^{s} \\
\Leftrightarrow & L_{\vec{r}} g(\vec{r}, Z)+(1 / 2) L_{Z} g(\vec{r}, \vec{r})=0 \text { along } \mathcal{Y}_{m}^{s} \\
\Leftrightarrow & L_{Z} g(\vec{r}, \vec{r})=0 \text { along } \mathcal{Y}_{m}^{s} \text { as, by definition of adapted } \\
& \text { coordinates, } Z \perp \mathcal{S}_{m}^{s} \text { so } g(\vec{r}, Z)=0 \text { along } \mathcal{S}_{m}^{s} \\
\Leftrightarrow & \frac{\mathrm{d}}{\mathrm{d} z}{ }_{\mid z=0} g_{z}^{s}(\vec{r}, \vec{r})=0 .
\end{aligned}
$$

To achieve the proof of (a), it remains to check that the datum of $\gamma$ on each submanifold $\left\{x=0, z=z_{0}\right\}$ and the fact that $\gamma(X)$ is $\left(\left(\check{g}_{z}\right)_{z \in I}, \lambda(\psi)\right)$-binded determine uniquely $\gamma$ on $\mathcal{M}$. It follows immediately from Definition 5.9 and from the fact that for each $z, \gamma_{z}$ is closed so $L_{X} \gamma\left(Y_{i}^{s}\right)=L_{Y_{i}^{s}} \gamma(X)$ for every $s \leqslant k$ and $i \leqslant n_{s}$. So (a) is proved.

Point (b) follows from the fact that, in adapted coordinates, the replacement of $X$ by $\mu X$ at the origin turns the coordinate $x$ into $\frac{1}{\mu} x$ and the coordinate $z$ into $\mu z$. 
Finally, for a given germ of metric, an adapted system of coordinates is determined by its data on $\{z=0\}$; if moreover you prescribe that the coordinate-vectors $Y_{i}^{s}$ are the exponential of linear orthonormal coordinate-vectors on $\mathbf{X}_{m}^{\perp}=\mathrm{T}_{m} \mathcal{X}_{0}^{\perp}$, an adapted system of coordinates is determined by the choice of the basis $\left(X,\left(\left(Y_{i}^{s}\right)_{i=1}^{n_{s}}\right)_{s=0}^{k}\right)$ of $\mathrm{T}_{m} \mathcal{X}_{0}^{\perp}$. The vector $X$ being fixed, for each $s$, the group $\mathrm{SO}\left(n_{s}, \mathbb{R}\right) \times \mathbb{R}^{n_{s}}$ acts simply transitively on the set of basis $\left(X,\left(Y_{i}^{s}\right)_{i=1}^{n_{s}}\right)$ of $\mathrm{T}_{m} \mathcal{Y}_{m}^{s}$, with $\left(Y_{i}^{s}\right)_{i=1}^{n_{s}}$ orthonormal: $\mathrm{SO}\left(n_{s}, \mathbb{R}\right)$ acts on the basis $\left(\check{Y}_{i}^{s}\right)_{i=1}^{n_{s}}$ of $\mathrm{T}_{m} \mathcal{Y}_{m}^{s} / \mathcal{X}_{m}$ and $\mathbb{R}^{n_{s}}$ on the choice of the supplement of $\mathbf{X}_{m}$ in $\mathbf{Y}_{m}^{s}=\mathrm{T} \mathcal{Y}_{m}^{s}$. In turn, the set $\left(X,\left(\left(Y_{i}^{s}\right)_{i=1}^{n_{s}}\right)_{s=0}^{k}\right)$ of those basis is the initial condition of the O.D.E. giving the adapted coordinates. Hence, for a given germ of metric, $\prod_{s=0}^{k} \mathrm{SO}\left(n_{s}, \mathbb{R}\right) \times \mathbb{R}^{n_{s}}$ acts simply transitively on the set of such adapted coordinates, so on the families $\left(\left(g_{z}^{s}\right)_{z \in]-\varepsilon, \varepsilon[}\right)_{s=0}^{k}$ and on $\gamma$. The orbits of this action correspond to equivalence classes of metrics, up to diffeomorphism. In other words, to germs are isometric if and only if they are given by two data $\left(\left(\left(g_{z}^{s}\right)_{z \in]-\varepsilon, \varepsilon[}\right)_{s=0}^{k}, \gamma\right)$ on the same orbit of this action. This is (c).

This action of $\prod_{s=0}^{k} \mathrm{SO}\left(n_{s}, \mathbb{R}\right) \times \mathbb{R}^{n_{s}}$, which is the natural action on the initial condition of an O.D.E., cannot be described explicitely on the solutions of the O.D.E. themselves, so

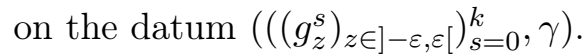

\section{Proof of Theorem 5.14 and of its adjacent results}

Proof of Lemma 5.11. By definition of the holonomy group, $\check{\mathfrak{h}}_{\check{m}}^{s} \subset \mathfrak{u}\left(\check{\mathbf{Y}}_{\check{m}}^{s}, g_{\tilde{m}}^{s}, J_{\tilde{m}}^{s}\right)$ if and only if $J_{\tilde{m}}^{s}$ can be locally extended as a parallel field of endomorphisms of $\dot{\mathbf{Y}}^{s}$. In turn this can be formulated as:

- each leaf $\check{\mathcal{Y}}_{\tilde{m}_{z}}^{s}$ is endowed with a parallel complex structure $J_{z}^{s}$,

- this $J_{z}^{s}$ is also parallel in the direction $Z$, i.e. $D_{Z} J_{z}^{s}=0$.

The first condition says simply that there exists a one-parameter family $J_{z}^{s}$ of complex structures of $\check{\mathcal{Y}}_{\check{m}_{z}}^{s}$, such that for all $z,\left(\check{\mathcal{Y}}_{\check{m}_{z}}^{s}, g_{z}^{s}, J_{z}^{s}\right)$ is Kähler. The latter is equivalent to Condition (5.3) of Definition 5.4. Indeed, let us take $i, j \in \llbracket 1, n_{s} \rrbracket$; besides we may suppose that the coordinates are complex, i.e. that or all $i, j,\left[J_{z}^{s} Y_{i}^{s}, Y_{j}^{s}\right]=0$. Moreover we propagate these coordinates by the flow of $Z$, so $\left[Z, Y_{i}^{s}\right]=0$. We denote by $Y$ and $Y^{\prime}$ any $Y_{i}^{s}$ and $Y_{j}^{s}$ and $J_{z}^{s}$ by $J$. Note that $J$ is parallel if and only if, for any $Y, D_{Z}(J Y)=J\left(D_{Z} Y\right)$. Now:

$$
\begin{aligned}
g\left(D_{Z}(J Y), Y^{\prime}\right)=\frac{1}{2}\left(L_{Z} g\left(J Y, Y^{\prime}\right)\right. & +\underbrace{L_{J Y} g\left(Z, Y^{\prime}\right)-L_{Y^{\prime}} g(Z, J Y)}_{=0 \text { as } \mathrm{d} \gamma=0} \\
& -g(Z, \underbrace{\left[J Y, Y^{\prime}\right]}_{=0})-g(J Y, \underbrace{\left[Z, Y^{\prime}\right]}_{=0})+\underbrace{g\left(Y^{\prime},[Z, J Y]\right)}_{=g\left(Y^{\prime},\left(L_{Z} J\right) Y\right) \text { as }[Z, Y]=0}) .
\end{aligned}
$$

A symmetric computation gives:

$$
g\left(J\left(D_{Z} Y\right), Y^{\prime}\right)=-g\left(D_{Z} Y, J Y^{\prime}\right)=\frac{1}{2}\left(-L_{Z} g\left(Y, J Y^{\prime}\right)+g\left(Y,\left(L_{Z} J\right) Y^{\prime}\right)\right) .
$$

As $g\left(Y, J Y^{\prime}\right)=-g\left(J Y, Y^{\prime}\right), L_{Z} J=\frac{\mathrm{d}}{\mathrm{d} z} J_{z}^{s}$ is $g_{z}^{s}$-selfadjoint if and only if $D_{Z} J_{z}^{s}=0$.

This proves the first part of Lemma 5.11. Let us deal with the second one. We now suppose that for every $z,\left(\check{\mathbf{Y}}_{\check{m}}^{s}, g_{\check{m}}^{s}\right)$ is Ricci-flat. By the Ambrose-Singer theorem, $\check{\mathfrak{h}}_{\check{m}}^{s} \not \subset$ $\mathfrak{s u}\left(\check{\mathbf{Y}}_{\check{m}}^{s}, g_{\grave{m}}^{s}, J_{\check{m}}^{s}\right)$ if and only if, at some point $p \in \mathcal{M}$, for some $A, B \in \mathrm{T}_{p} \mathcal{M}, R(A, B)_{\mid \check{\mathbf{Y}}_{p}^{s}}$ has nonvanishing $J^{s}$-complex trace, i.e. $\operatorname{tr}_{g^{s}}\left(g\left(R(A, B)_{\mid \check{\mathbf{Y}}_{p}^{s}} \cdot, J^{s} \cdot\right)\right) \neq 0$, with $\operatorname{tr}_{g^{s}}$ the real 
trace of a real bilinear form, with respect to the metric $g^{s}$. Now if $A$ or $B$ is in ${ }_{r \neq s} \mathbf{Y}_{p}^{r}$, by Lemma 4.1, $R(A, B)_{\check{\mathbf{Y}}_{p}^{s}}=0$. Besides, for every $z, g_{s}^{z}$ is supposed to be Ricci-flat, i.e. precisely, if $A, B \in \mathbf{Y}_{p}^{s}$, the $J^{s}$-complex trace of $R(A, B)_{\mid \check{\mathbf{Y}}_{p}^{s}}$ vanishes. So if this trace is nonzero for some $A, B \in \mathrm{T}_{p} \mathcal{M}$, it is with $B=Z$ and $A \in \mathbf{Y}_{p}^{s}$. But now, by Proposition 3.12 :

$$
\left.\left.\operatorname{tr}_{g^{s}}\left(g\left(R(A, Z)_{\mid \check{\mathbf{Y}}_{p}^{s}} \cdot, J^{s} \cdot\right)\right)=\operatorname{tr}_{g^{s}}\left(g\left(R\left(\cdot, J^{s} \cdot\right) A, Z\right)\right)=\operatorname{tr}_{g^{s}}\left(\mathrm{~d}^{D_{z}^{s}} \frac{\mathrm{d} g_{z}^{s}}{\mathrm{~d} z}\right)\left(\cdot, J^{s} \cdot\right) A\right)\right),
$$

which proves Lemma 5.11.

Proof of Proposition 5.6. (a) We use the notation of Remark 5.5. In local coordinates, we set $G_{t}=\operatorname{Mat}\left(g_{t}\right), \underline{G}_{t}=\operatorname{Mat}\left(\underline{g}_{t}\right), P_{t}=\operatorname{Mat}\left(\mathrm{d} \varphi_{t}\right)$ and identify $J_{t}$ with its matrix; a prime after a symbol stands for its derivative in $t .{ }^{t} M$ stands for the transpose of a matrix $M$. Then:

$J_{t}^{\prime} \quad$ is $g_{t}$-selfadjoint

$\Leftrightarrow G_{t} J_{t}^{\prime} \quad$ is symmetric

$\Leftrightarrow\left({ }^{t} P_{t}^{-1} \underline{G}_{t} P_{t}^{-1}\right)\left(P_{t}^{\prime} J_{0} P_{t}^{-1}-P_{t} J_{0} P_{t}^{-1} P_{t}^{\prime} P_{t}^{-1}\right) \quad$ is symmetric

$\Leftrightarrow \underline{G}_{t} J_{0} P_{t}^{-1} P_{t}^{\prime}-\underline{G}_{t} P_{t}^{-1} P_{t}^{\prime} J_{0}$ is symmetric (multiply left by ${ }^{t} P_{t}$, right by $P_{t}$ )

$\Leftrightarrow-{ }^{t} J_{0} \underline{G}_{t} P_{t}^{-1} P_{t}^{\prime}-\underline{G}_{t} P_{t}^{-1} P_{t}^{\prime} J_{0}$ is symmetric, as $J_{0}$ is $\underline{g}_{t}$-skew-adjoint,

$\Leftrightarrow{ }^{t} J_{0} A_{t}+A_{t} J_{0}={ }^{t} A_{t} J_{0}+{ }^{t} J_{0}^{t} A_{t} \quad$ is symmetric, with $A_{t}=\underline{G}_{t} P_{t}^{-1} P_{t}^{\prime}$

$\Leftrightarrow{ }^{t} J_{0}\left(A_{t}-{ }^{t} A_{t}\right)=-\left(A_{t}-{ }^{t} A_{t}\right) J_{0}$

$\Leftrightarrow \alpha_{t}\left(J_{0} \cdot, \cdot\right)=-\alpha_{t}\left(\cdot, J_{0} \cdot\right)$, with $\alpha_{t}$ the 2 -form the matrix of which is $A_{t}-{ }^{t} A_{t}$

$\Leftrightarrow \alpha_{t}$ is of type $(1,1)$, with respect to the complex structure $J_{0}$.

Claim. $\alpha_{t}=\mathrm{d} w_{t}^{b^{t}}$, with $\underline{b}^{t}$ the musical isomorphism associated with $\underline{g}_{t}$.

Indeed, let us choose a point $p \in \mathcal{M}$, any basis $\beta$ of $T_{p} \mathcal{M}$ and let us prove that $\operatorname{Mat}_{\beta}\left(\mathrm{d} w_{t}^{b_{t}}\right)=$ $A_{t}-{ }^{t} A_{t}=\underline{G}_{t} P_{t}^{-1} P_{t}^{\prime}-{ }^{t}\left(\underline{G}_{t} P_{t}^{-1} P_{t}^{\prime}\right)$. By the $\underline{g}_{t}$-exponential map, $\beta$ gives a system of $\underline{g}_{t}$-normal coordinates in a neighbourhood of $p$. Let $B$ and $C$ be two normal coordinate-vectors.

$$
\begin{aligned}
\mathrm{d} w_{t}^{b_{t}}(B, C)= & L_{B}\left(\underline{g}_{t}\left(w_{t}, C\right)\right)-L_{C}\left(\underline{g}_{t}\left(w_{t}, B\right)\right) \\
= & \left(L_{B} \underline{g}_{t}\right)\left(w_{t}, C\right)+\underline{g}_{t}\left(\left[B, w_{t}\right], C\right)+\underline{g}_{t}\left(w_{t},[B, C]\right) \\
& \quad-\left(L_{C} \underline{g}_{t}\right)\left(w_{t}, B\right)-\underline{g}_{t}\left(\left[C, w_{t}\right], B\right)-\underline{g}_{t}\left(w_{t},[C, B]\right) .
\end{aligned}
$$

Now $[B, C]=0$ and, at $p, L_{B} \underline{g}_{t}=L_{C} \underline{g}_{t}=0$. Besides:

$$
\begin{aligned}
& {\left[B, w_{t}\right] }=L_{B}\left(\frac{\mathrm{d}}{\mathrm{d} s} \mid s=t\right. \\
&=\left.\frac{\mathrm{d}}{\mathrm{d} s}\right|_{s=t} ^{-1}\left(\varphi_{B}\left(\varphi_{t}^{-1} \varphi_{s}\right)\right) \\
&=\left.\frac{\mathrm{d}}{\mathrm{d} s}\right|_{s=t}\left(\mathrm{~d} \varphi_{t}^{-1} \cdot \mathrm{d} \varphi_{s} \cdot B\right) \\
&=\mathrm{d} \varphi_{t}^{-1} \cdot\left(\frac{\mathrm{d}}{\mathrm{d} s} \mid s=t\right. \\
&\left.\mathrm{d} \varphi_{s}\right) \cdot B
\end{aligned}
$$

which is exactly saying that at $p, \operatorname{Mat}_{\beta}\left(\left[B, w_{t}\right]\right)=P_{t}^{-1} P_{t}^{\prime} \operatorname{Mat}_{\beta}(B)$. As $\mathrm{d} w_{t}^{b_{t}}$ is a tensor, we can replace in (6.1), and obtain an equality only depending on the value of $B$ and $C$ at $p$. 
This gives $\operatorname{Mat}_{\beta}\left(\mathrm{d} w_{t}^{b_{t}}\right)=\underline{G}_{t} P_{t}^{-1} P_{t}^{\prime}-{ }^{t}\left(\underline{G}_{t} P_{t}^{-1} P_{t}^{\prime}\right)$, which proves the claim. Therefore:

$$
\left(g_{t}, J_{t}\right)_{t \in I} \text { satisfy (5.3) }
$$

$\Leftrightarrow \mathrm{d} W_{t}^{\frac{b^{t}}{t}}$ is of $J_{0}$-type $(1,1)$

$\Leftrightarrow \mathrm{d}\left(\omega^{(1,0)}+\omega^{(0,1)}\right)$ is of $J_{0}$-type $(1,1)$, setting $W_{t}^{\underline{b}^{t}}=\omega^{(1,0)}+\omega^{(0,1)}$

$\Leftrightarrow \underbrace{\partial \omega^{(1,0)}}_{\text {type }(2,0)}+\underbrace{\partial \omega^{(0,1)}+\bar{\partial} \omega^{(1,0)}}_{\text {type }(1,1)}+\underbrace{\bar{\partial} \omega^{(0,1)}}_{\text {type }(0,2)}$ is of $J_{0}$-type $(1,1)$

$\Leftrightarrow \partial \omega^{(1,0)}=\bar{\partial} \omega^{(0,1)}=0$

$\Leftrightarrow \partial \omega^{(1,0)}=0 \quad$ as, $W_{t}^{b^{t}}$ being real, $\omega^{(0,1)}=\overline{\omega^{(1,0)}}$

$\Leftrightarrow \omega^{(1,0)}=\partial f_{t}$, where $f_{t}$ is a complex function, as $\partial$ is locally exact

$\Leftrightarrow W_{t}^{\underline{b}^{t}}=\partial f_{t}+\overline{\partial f_{t}}=\mathrm{d}(\Re f)+(\mathrm{d}(\Im f)) \circ J_{0} \quad$ which proves (a).

6.1 Remarks • About the meaning of the last line, see also Remark 5.7 p. 19.

- We can check the coherence of the result with the particular case $J_{t}=J_{0}$ for all $t$. Then $\frac{\mathrm{d}}{\mathrm{d} t} J_{t}=0$ so $\left(J_{t}, g_{t}\right)_{t \in I}$ satisfies (5.3); besides $\left(\varphi_{t}\right)_{t \in I}$ is a family of $J_{0}$-biholomorphisms. It is the case if and only if $W_{t}$, or equivalently $V_{t}$, is a family of holomorphic vector fields, which can be checked to be equivalent to $W_{t}^{b^{t}}=\partial f_{t}+\overline{\partial f_{t}}$ with $f_{t}$ holomorphic from $\left(\mathbb{R}^{2 N}, J_{0}\right)$ to $\mathbb{C}$. So, considering $\varphi_{t}$, as in 5.8 , as defined up to right composition by a $J_{0}$-biholomorphism, is equivalent to considering $f_{t}$ in the same way.

(b) We have to find one admissible and Ricci-flat family $\left(g_{t}, J_{t}\right)_{t \in I}$. In fact, we exhibit a non-empty collection of such families. Let us choose $\left(g_{0}, J_{0}\right)$ any Ricci-flat Kähler metric, $\left(f_{t}\right)_{t \in I}$ some family of functions $f_{t} \in C^{\infty}(\mathcal{M}, \mathbb{C})$ and $\left(\varphi_{t}\right)_{t \in I}$ the family of diffeomorphisms associated with it as above; we set $g_{t}=\left(\varphi_{t}^{-1}\right)^{*} g_{0}$. By Lemma 6.4, independent of the rest of the paper, with $\underline{g}_{t} \equiv g_{0}, b_{t}$ the musical isomorphism associated with $g_{t}, D_{t}$ its Levi-Civita connection, $R_{t}$ its curvature and $V_{t}$ the vector field such that $\varphi_{t}$ is the flow of $V_{t}$ :

$$
\mathrm{d}^{D_{t}}\left(\frac{\mathrm{d} g_{t}}{\mathrm{~d} t}\right)(A, B, C)=-D_{t}\left(\mathrm{~d} V_{t}^{b_{t}}\right)(C, A, B)+2 g_{t}\left(R_{t}\left(C, V_{t}\right) A, B\right)
$$

So:

$$
\begin{aligned}
\left.\operatorname{tr}_{g_{t}}\left(\mathrm{~d}^{D_{t}}\left(\frac{\mathrm{d} g_{t}}{\mathrm{~d} t}\right)\left(\cdot, J_{t} \cdot, U\right)\right)\right) & =-\operatorname{tr}_{g_{t}}\left(D_{t}\left(\mathrm{~d} V_{t}^{\mathrm{b}_{t}}\right)\left(U, \cdot, J_{t} \cdot\right)\right)-2 \operatorname{tr}\left(R_{t}\left(C, V_{t}\right) \circ J_{t}\right) \\
& =-L_{U} \operatorname{tr}_{g_{t}}\left(\mathrm{~d} V_{t}^{b_{t}}\left(\cdot, J_{t} \cdot\right)\right)-2 \operatorname{tr}\left(R_{t}\left(C, V_{t}\right) \circ J_{t}\right)
\end{aligned}
$$

As $g_{0}$ is Ricci-flat, so are every $g_{t}$, so $\operatorname{tr}\left(R_{t}\left(C, V_{t}\right) \circ J_{t}\right)=0$. So $\left(g_{t}, J_{t}\right)_{t \in I}$ satisfy (5.4) if and only if $\operatorname{tr}_{g_{t}}\left(\mathrm{~d} V_{t}^{b_{t}}\left(\cdot, J_{t} \cdot\right)\right)$ is not constant over $\varphi_{t}(\mathcal{M})$, i.e. $\operatorname{tr}_{\underline{g}_{t}}\left(\mathrm{~d} W_{t}^{\underline{\mathrm{b}}_{t}}\left(\cdot, J_{0} \cdot\right)\right)$ is not constant over $\mathcal{M}$. Now:

Claim. $\operatorname{tr}_{\underline{g}_{t}}\left(\mathrm{~d} W_{t}^{\underline{\mathrm{b}}_{t}}\left(\cdot, J_{0} \cdot\right)\right)=\Delta_{\underline{g}_{t}}\left(\Im f_{t}\right)$.

Therefore, in our case, $\left(g_{t}, J_{t}\right)_{t \in I}$ satisfies (5.4) if and only if $\Im f_{t}$ has not a constant $\underline{g}_{t}$-laplacian. (Lots of) germs of such functions exist, so we are left with proving the claim.

$$
\begin{aligned}
\mathrm{d} W_{\bar{t}}^{\underline{\underline{t}}^{t}} & =(\partial+\bar{\partial})\left(\partial f_{t}+\bar{\partial} \overline{f_{t}}\right) \\
& =\bar{\partial} \partial f_{t}+\partial \bar{\partial} \overline{f_{t}} \\
& =(\bar{\partial} \partial+\partial \bar{\partial})\left(\Re f_{t}\right)-\mathrm{i}(\bar{\partial} \partial-\partial \bar{\partial})\left(\Im f_{t}\right) \\
& =2 \mathrm{i} \partial \bar{\partial}\left(\Im f_{t}\right) \quad \text { as } \bar{\partial} \partial+\partial \bar{\partial}=0 .
\end{aligned}
$$


Eventually, for a Kähler metric, $\Delta_{\underline{g}_{t}}\left(\Im f_{t}\right)$ is equal to the symplectic trace of $2 \mathrm{i} \partial \bar{\partial}\left(\Im f_{t}\right)$, i.e. precisely to $\operatorname{tr}_{\underline{g}_{t}}\left(\mathrm{~d} W_{t}^{\underline{\mathrm{b}}^{t}}\left(\cdot, J_{0} \cdot\right)\right)$, see e.g. [LB70], p. 32. We are done.

Proof of Proposition 5.13. The existence of the complex structures $J^{s}$ is given by Lemma 5.1. Now, as defined in Theorem $2.1, \mathfrak{h}$ is of type 3 with $k^{\prime}$-tuple $\left(\lambda^{s}\right)_{s=1}^{k^{\prime}}$ if and only if:

$$
\forall H \in \mathfrak{h}_{m}, g(H(X), Z)=\sum_{s=1}^{k^{\prime}}\left(\operatorname{tr}_{J^{s}} H_{\mid \check{\mathbf{Y}}^{s}}\right) \lambda^{s} \quad \text { and: } \forall s \in \llbracket 1, k^{\prime} \rrbracket, \operatorname{tr}_{J^{s}} H_{\mid \check{\mathbf{Y}}^{s}} \neq 0,
$$

i.e., by the Ambrose-Singer theorem, if and only if:

$$
\forall p \in \mathcal{M}, \forall A, B \in \mathrm{T}_{p} \mathcal{M}, g(R(A, B) X, Z)=\sum_{s=1}^{k^{\prime}}\left(\operatorname{tr}_{J^{s}} R(A, B)_{\mid \check{\mathbf{Y}}^{s}}\right) \lambda^{s} \quad \text { and }
$$

for every $s \in \llbracket 1, k^{\prime} \rrbracket$, there is a point $q, A, B \in \mathrm{T}_{q} \mathcal{M}$ with: $\operatorname{tr}_{J^{s}} R(A, B)_{\mid \check{\mathbf{Y}}^{s}} \neq 0$.

By Lemma 4.1, $A, B \in \mathbf{X}_{p}^{\perp} \Rightarrow R(A, B) X=0$ and, if $r \neq s,\left(A \in \mathbf{Y}^{r}\right.$ or $\left.B \in \mathbf{Y}^{r}\right) \Rightarrow$ $R(A, B)_{\mid \mathbf{Y}^{s}}=0$. So (6.6) is equivalent to:

$$
\begin{aligned}
& \forall p \in \mathcal{M}, \forall s \in \llbracket 0, k \rrbracket, \forall Y \in \mathbf{Y}_{p}^{s}, g(R(Y, Z) X, Z)=\sum_{r=1}^{k^{\prime}}\left(\operatorname{tr}_{J^{s}} R(Y, Z)_{\left.\right|_{\mathbf{Y}^{r}}}\right) \lambda^{r} \\
& =\left\{\begin{array}{l}
\left(\operatorname{tr}_{J^{s}} R(Y, Z)_{\mid \check{\mathbf{Y}}^{s}}\right) \lambda^{s} \text { if } s \in \llbracket 1, k^{\prime} \rrbracket \\
0 \text { else }
\end{array}\right.
\end{aligned}
$$

Proposition 5.13's part dealing with type 3 algebras follows. The second part is similar.

Proof of Theorem 5.14. (a), first claim. The algebra $\mathfrak{h}$ is of type 2 or 4 if and only if the holonomy group acts trivially on $\mathbf{X}$. In turn, this is equivalent to the fact that, along any leaf of $\mathcal{X}^{\perp}$, the form $\alpha_{\sigma(f), Z, Z}$ introduced in Lemma 4.3 is the pull back $\pi^{*} \check{\alpha}$ of a one-form $\check{\alpha}$ defined on $\check{\mathcal{X}}^{\perp}$. Now by Remark 4.13, $L_{Z} \gamma=\alpha_{\sigma(f), Z, Z}$ and, on $\{z=0\}, \gamma=\mathrm{d} x$.

(b) We now show (b), postponing the end of the proof of (a). We have to translate Proposition 5.13 in terms of properties of the metric, in adapted coordinates. This is nothing but putting together the previous results. By Proposition 3.4 applied to the terms of the type $g(R(*, Z) *, Z)$ and Proposition 3.12 applied to those of the type $\operatorname{tr}_{J^{s}} R(*, *)$ and as, for a $J^{s}$-complex endomorphism $A, \operatorname{tr}_{J^{s}} A=\operatorname{tr}_{\check{g}^{s}} g\left(\cdot, J^{s} \cdot\right),(5.9)$ is equivalent to, at any $p$ :

$$
\left\{\begin{aligned}
& s=0 \text { or } s>k^{\prime} \Rightarrow \forall Y \in \mathbf{Y}^{s}, L_{Z} L_{Y}(\ln \gamma(X))=0 \\
& 1 \leqslant s \leqslant k^{\prime} \Rightarrow \quad \forall Y \in \mathbf{Y}^{s}, \gamma(X) L_{Z} L_{Y}(\ln \gamma(X))=\operatorname{tr}_{\check{g}_{z}^{s}}\left(\mathrm{~d}^{D_{t}^{s}}\left(\frac{\mathrm{d} \check{g}_{z}^{s}}{\mathrm{~d} z}\right)\left(\cdot, J_{z}^{s} \cdot, Y\right)\right) \lambda^{s} \\
& \text { and } \forall Y, Y^{\prime} \in \mathbf{Y}^{s}, \operatorname{tr}_{J_{z}^{s}}\left(R\left(Y, Y^{\prime}\right)_{\mid \check{\mathbf{Y}}^{s}}\right)=0 .
\end{aligned}\right.
$$

By the Ambrose-Singer theorem, points (5.7) and (5.10) of Proposition 5.13 imply that for each $s \in \llbracket 1, k^{\prime} \rrbracket, \check{\mathfrak{h}}_{\check{m}}^{s} \subset \mathfrak{u}\left(\check{\mathbf{Y}}_{\check{m}}^{s}, g_{\check{m}}^{s}, J_{\check{m}}^{s}\right)$ and $\check{\mathfrak{h}}_{\check{m}}^{s} \not \subset \mathfrak{s u}\left(\check{\mathbf{Y}}_{\check{m}}^{s}, g_{\check{m}}^{s}, J_{\check{m}}^{s}\right)$. In particular, for $s \in \llbracket 1, k^{\prime} \rrbracket$, each $\check{g}_{z}^{s}$ is Kähler. Moreover, again by the the Ambrose-Singer theorem applied to each leaf of $\check{\mathcal{Y}}^{s}$ for $s \in \llbracket 1, k^{\prime} \rrbracket$, the last point of (6.8) holds if and only if for each $z$, the metric $\check{g}_{z}^{s}$ is Ricci-flat. So, by Lemma 5.11, Properties (5.7) and (5.10) and the last point of (6.8) hold if and only if for each $s \in \llbracket 1, k^{\prime} \rrbracket,\left(J_{z}^{s},\left(\check{g}_{z}^{s}\right)_{z \in I}\right)$ is Ricci flat, admissible.

Besides, both first claims of (6.8) correspond exactly to the fact that the function $\gamma(X)$ is $\left(\left(\check{g}_{z}\right)_{z \in I}, \lambda\right)$-binded, see Definition 5.9. 
We are left with showing that, for a given $\left(\check{g}_{z}\right)_{z \in I}=\left(\prod_{s=0}^{k} \check{g}_{z}^{s}\right)_{z \in I}$ such that, for each $s \in$ $\llbracket 1, k^{\prime} \rrbracket,\left(\check{g}_{z}^{s}\right)_{z \in I}$ is Ricci-flat and admissible, and for each given $k^{\prime}$-tuple $\lambda=\left(\lambda^{s}\right)_{s=1}^{k^{\prime}} \in\left(\mathbb{R}^{*}\right)^{k^{\prime}}$, one and only one function $f$ on $\mathcal{M}$, equal to 1 on $\mathcal{X}_{m}^{\perp}$ and along $\left(m_{z}\right)_{z \in I}$, is $\left(\left(\check{g}_{z}\right)_{z \in I}, \lambda\right)$ binded. We need the following lemma, proven just after the present proof's end.

6.2 Lemma Let $s$ be in $\llbracket 1, k^{\prime} \rrbracket$. The one-form $\tau_{z}: Y \mapsto \sum_{s=1}^{k^{\prime}} \lambda^{s} \operatorname{tr}_{J^{s}}\left(R(Y, Z)_{\mid \check{\mathbf{Y}}^{s}}\right)$, defined along any leaf $\mathcal{X}_{z}^{\perp}$ of $\mathcal{X}^{\perp}$, is closed.

Now, with $F=\ln f$, defined at least for small values of $z$ :

$f$ is $\left(\left(\check{g}_{z}\right)_{z \in I}, \lambda\right)$-binded $\Leftrightarrow \forall p, \mathrm{~d}\left(\frac{\mathrm{d} F}{\mathrm{~d} z}\right)_{\mid \mathbf{X}^{\perp}}=e^{-F} \tau_{z}, \quad$ with $\tau_{z}$ the one-form introduced in Lemma 6.2 $\Leftrightarrow\left(L_{Z} F\right)_{\mid \mathbf{X}^{\perp}}\left(=\frac{\mathrm{d} F}{\mathrm{~d} z} \mid \mathbf{X}^{\perp}\right)=\mathcal{I}\left(e^{-F} \tau_{z}\right)$

with $\mathcal{I}$ the operator associating, with a closed one-form $\alpha$ on $\mathcal{X}_{z}^{\perp}$, its integral vanishing at $m_{z}$. For any $r \geqslant 1, \mathcal{I}$ is continuous and even Lipschitzian on $C^{r}$, for the $C^{r}$ norms. Besides, (6.9) makes sense, i.e. is an O.D.E., in the Banach space $E^{r}=\left\{F \in C^{r}\left(I^{n-1}, \mathbb{R}\right) ; F(0)=\right.$ 0 and $e^{-F} \tau_{z}$ is closed $\}$. Indeed, the map $F \mapsto \mathcal{I}\left(e^{-F} \tau_{z}\right)$, maps $E^{r}$ in $E^{r}$ :

$$
\begin{aligned}
\mathrm{d}\left(e^{-\mathcal{I}\left(e^{-F} \tau_{z}\right)} \tau_{z}\right) & =\left(-e^{-\mathcal{I}\left(e^{-F} \tau_{z}\right)} \mathrm{d}\left(\mathcal{I}\left(e^{-F} \tau_{z}\right)\right) \wedge \tau_{z} \quad \text { as } \tau_{z} \text { is closed, by Lemma } 6.2\right. \\
& =\left(-e^{-\mathcal{I}\left(e^{-F} \tau_{z}\right)} e^{-F} \tau_{z}\right) \wedge \tau_{z} \\
& =0
\end{aligned}
$$

So, by the Cauchy Lipschitz theorem applied in $E^{r}$, (6.9) admits a unique solution with value $F \equiv 0$ at $z=0$; as it is thus of class $C^{r}$ for all $r$, it is of class $C^{\infty}$. We are done.

(a), end. The arguments of (b) applied to each function $\gamma\left(Y_{i}^{0}\right), 1 \leqslant i \leqslant n_{0}^{\prime}$ with $\left(Y_{i}^{0}\right)_{i=1}^{n_{0}^{\prime}}$ the coordinate-vectors spanning $\mathbf{Y}^{0 \prime}$, give the result. The last step, using Lemma 6.2 , is even simpler, as the O.D.E. one gets is $L_{Z} f=\mathcal{I}\left(\tau_{z}\right)$. So we omit the details.

Proof of Lemma 6.2. In fact, for each $s \in \llbracket 1, k^{\prime} \rrbracket, Y \mapsto \operatorname{tr}_{J^{s}}\left(R(Y, Z)_{\mid \mathbf{Y}^{s}}\right)$ is closed. Notice that, as $\check{g}^{s}$ is Ricci-flat, $\operatorname{tr}_{J^{s}}\left(R\left(\mathbf{Y}^{s}, \mathbf{Y}^{s}\right)_{\mid \mathbf{Y}^{s}}\right)=\{0\}$; besides, by Lemma 4.1, for $r \neq s$, $R\left(\mathbf{Y}^{r}, \mathbf{Y}^{r}\right)_{\mid \mathbf{Y}^{s}}=\{0\}$, so $\operatorname{tr}_{J^{s}}\left(R\left(\mathbf{X}^{\perp}, \mathbf{X}^{\perp}\right)_{\mid \mathbf{Y}^{s}}\right)=\{0\}$. Let $Y, Y^{\prime}$ be two adapted coordinatevectors of $\mathbf{X}^{\perp}$. We denote below by $\Sigma$ and $\Sigma^{\prime}$ some sums of terms $\operatorname{in}_{J^{s}}\left(R\left(\mathbf{X}^{\perp}, \mathbf{X}^{\perp}\right)_{\mid \mathbf{Y}^{s}}\right)$, thus vanishing.

$$
\begin{aligned}
& L_{Y} \operatorname{tr}_{J^{s}}\left(R\left(Y^{\prime}, Z\right)_{\mid \mathbf{Y}^{s}}\right)-L_{Y^{\prime}} \operatorname{tr}_{J^{s}}\left(R(Y, Z)_{\mid \mathbf{Y}^{s}}\right) \\
= & \operatorname{tr}_{J^{s}}\left(\left(D_{Y} R\right)\left(Y^{\prime}, Z\right)_{\mid \mathbf{Y}^{s}}-\left(D_{Y^{\prime}} R\right)(Y, Z)_{\mid \mathbf{Y}^{s}}\right)+\Sigma \\
= & \operatorname{tr}_{J^{s}}\left(\left(D_{Z} R\right)\left(Y^{\prime}, Y\right)_{\mid \mathbf{Y}^{s}}\right) \quad \text { by the second Bianchi identity } \\
= & L_{Z} \operatorname{tr}_{J^{s}}\left(R\left(Y^{\prime}, Y\right)_{\mid \mathbf{Y}^{s}}\right)+\Sigma^{\prime} \\
= & 0 .
\end{aligned}
$$

The last remaining work is to state and prove Lemma 6.4, independent of the rest of the article. We need the following definition.

6.3 Definition If $A$ is a $(p, q)$-tensor on a manifold $\mathcal{M}$ with a connection $D, \delta^{*} A$ is the symmetrization of $D A$, i.e. the $(p+1, q)$-tensor defined by:

$$
\delta^{*} A\left(X_{1}, \ldots, X_{p+1}\right)=\frac{1}{(p+1) !} \sum_{\sigma \in \mathfrak{S}_{p+1}} D A\left(X_{\sigma(1)}, \ldots, X_{\sigma(p+1)}\right)
$$


6.4 Lemma Let $\left(\varphi_{t}\right)_{t \in I}$ be a one-parameter family of diffeomorphisms from an open set $\mathcal{U}$ of $\mathbb{R}^{n}$ to $\varphi_{t}(\mathcal{U}),\left(V_{t}\right)_{t \in I}$ the one-parameter family of vector fields on $\varphi_{t}(\mathcal{U})$ such that $\frac{\mathrm{d}}{\mathrm{d} t} \varphi_{t}=V_{t} \circ \varphi_{t}$ and $\left(\underline{g}_{t}\right)_{t \in I}$ a family of Riemannian metrics on $\mathcal{U}$. We set $g_{t}=\left(\varphi_{t}^{-1}\right)^{*} \underline{g}_{t}$, and denote by $D_{t}$ the Levi-Civita connection of $g_{t}$, by $\delta_{t}^{*}$ its symmetrization, by $R_{t}$ its curvature tensor and by $b_{t}$ musical isomorphism of $g_{t}$. Then:

$$
\begin{gathered}
\frac{\mathrm{d}}{\mathrm{d} t} g_{t}=-2 \delta_{t}^{*}\left(V_{t}^{\mathrm{b} t}\right)+\left(\varphi_{t}^{-1}\right)^{*} \frac{\mathrm{d}}{\mathrm{d} t} \underline{g}_{t}, \\
\mathrm{~d}^{D_{t}}\left(\frac{\mathrm{d} g_{t}}{\mathrm{~d} t}\right)(A, B, C)=2 g_{t}\left(R_{t}(A, B) C, V_{t}\right)-D_{t}\left(\mathrm{~d} V_{t}^{b_{t}}\right)(C, A, B)+\mathrm{d}^{D_{t}}\left(\left(\varphi_{t}^{-1}\right)^{*}\left(\frac{\mathrm{d} \underline{g}_{t}}{\mathrm{~d} t}\right)\right)(A, B, C) .
\end{gathered}
$$

Proof. (6.11) is standard and follows from Lemma 1.60 p. 35 of [Bes87]. For (6.12), we may suppose that $\underline{g}_{t}$ is constant; we also drop everywhere the index $t$. Take $A, B, C$ any three normal coordinate-vectors at any point $p$. At $p, D_{A} B=D_{B} A=D_{A} C=\ldots=0$, so:

$$
\begin{aligned}
-D\left(\frac{\mathrm{d} g}{\mathrm{~d} t}\right)(A, B, C) & =-L_{A}\left(\frac{\mathrm{d} g}{\mathrm{~d} t}\right)(B, C) \\
& =2 L_{A} \delta^{*}\left(V^{b}\right)(B, C) \quad \text { by }(6.11) \\
& =L_{A} g\left(D_{B} V, C\right)+L_{A} g\left(D_{C} V, B\right) \\
& =g\left(D_{A} D_{B} V, C\right)+L_{A} L_{C} g(V, B)-L_{A} g\left(V, D_{C} B\right) \\
& =g\left(D_{A} D_{B} V, C\right)+L_{C} L_{A} g(V, B)-g\left(V, D_{A} D_{C} B\right) \\
& =g\left(D_{A} D_{B} V, C\right)+L_{C} L_{A} V^{b}(B)-g\left(V, D_{A} D_{B} C\right) .
\end{aligned}
$$

So: $\quad-\mathrm{d}^{D}\left(\frac{\mathrm{d} g}{\mathrm{~d} t}\right)(A, B, C)=g(R(A, B) V, C)+L_{C}\left(\mathrm{~d} V^{\mathrm{b}}(A, B)\right)-g(R(A, B) C, V)$

$$
=-2 g(R(A, B) C, V)+\left(D_{C} \mathrm{~d} V^{b}\right)(A, B) \text {. }
$$

\section{Additional comments}

\section{7. a A parametrization of the set of germs of Lorentzian reducible metrics}

We parametrize, using Theorem 3.7, the set of germs of Lorentzian reducible-indecomposable metrics, and by the way, understand them a bit better. To obtain such a metric, one must first choose the parallel distributions $\left(\mathbf{X},\left(\mathbf{Y}^{s}\right)_{s=0}^{k}\right)$. So in this paragraph, $\left(\mathbf{X},\left(\mathbf{Y}^{s}\right)_{s=0}^{k}\right)$ are subspaces of $\mathbb{R}^{n}$ such that $\mathbf{X}=\mathbb{R} \times\{0\}^{n-1}, \operatorname{dim}\left(+_{s=0}^{k} \mathbf{Y}^{s}\right)=n-1$, for all $s, \mathbf{X} \subset \mathbf{Y}^{s}$ and $\oplus_{s=0}^{k}\left(\mathbf{Y}^{s} / \mathbf{X}\right)=\mathbb{R}^{n} / \mathbf{X}$. We set $n_{s}=\operatorname{dim} \mathbf{Y}^{s} / \mathbf{X}$. We focus on the germs at $0 \in \mathbb{R}^{n}$ of Lorentzian metrics $g$ such that, identifying $\mathbb{R}^{n}$ with the tangent space at the origin and denoting by $\mathfrak{h}$ the holonomy algebra of $g$ :

$$
g(\mathbf{X}, \mathbf{X})=\{0\},+_{s=0}^{k} \mathbf{Y}^{s}=\mathbf{X}^{\perp}, \mathfrak{h} \text { stabilizes }\left(\mathbf{X},\left(\mathbf{Y}^{s}\right)_{s=1}^{k}\right) \text { and acts trivially on } \mathbf{Y}^{0} .
$$

7.1 Recall If $Z$ is any vector such that $g(Z, X)=1$ and $\left(Y, Y^{\prime}\right) \in \mathbf{Y}^{s} \times \mathbf{Y}^{r}$ with $r \neq s$ or $r=s=0$, then the quantity $g\left(R(Y, Z) Y^{\prime}, Z\right)$ is independent of the choice of $Z$, it follows from Lemma 4.1.

7.2 Corollary [of Theorem 3.7] Let $g$ be a germ of Lorentzian metric satisfying (7.1). Then $g$ is given by the independent and arbitrary data of:

(i) A nonzero vector $X$ of $\mathbf{X}$ at the origin,

(ii) The quotient metrics $\left(\left(\check{g}_{z}^{s}\right)_{z \in I}\right)_{s=1}^{k}$ it induces on $\left(\left(\check{\mathcal{Y}}_{z}^{s}\right)_{s=1}^{k}\right)_{z \in I}$, given as one-parameter families of germs of Riemannian metrics on $\mathbb{R}^{n_{s}}$, 
(iii) A function $f \in C^{\infty}\left(\mathbb{R}^{n}, \mathbb{R}\right)$. This function satisfies, at any point, for any $Z$ such that $g(Z, X)=1$, for all $Y, Y^{\prime}$ in $\mathbf{Y}^{s} \times \mathbf{Y}^{r}$ with $r \neq s$ or $r=s=0, L_{Y} L_{Y^{\prime}} f=L_{Y^{\prime}} L_{Y} f=$ $g\left(R(Y, Z) Y^{\prime}, Z\right)=: \beta\left(Y, Y^{\prime}\right)$. See Proposition 7.4 and Remark 7.5 (ii) below for details. In fact besides, $f$ is naturally defined up to addition of a function of the form $\sum_{s=1}^{k} f^{s}, f^{s}$ depending only on the coordinates $\left(y^{s}\right)_{s=1}^{k}$ and $z$.

If $X$ is turned into $\mu X, \mu \in \mathbb{R}^{*}$, the same germ $g$ is given by (ii) the families $\left(\check{g}_{\frac{1}{\mu}} z\right)_{z \in \mu I}$ and (iii) the function $f_{\mu}:\left(x,\left(y^{s}\right)_{s=1}^{k}, z\right) \mapsto \frac{1}{\mu} f\left(\mu x,\left(y^{s}\right)_{s=1}^{k}, \frac{1}{\mu} z\right)$.

The vector $X$ chosen in (i) being fixed, two data of (ii) +(iii) give the same germ $g$, up to diffeomorphism, if and only if for each $s \geqslant 1$, both families $\left(\check{g}_{z}^{s}\right)_{z \in I}$ are on the same orbit of an action of $\mathcal{G}\left(\mathbb{R}^{n_{s}}, 0\right) \times \mathcal{G}\left(\left(\mathbb{R}^{n_{s}}, \mathbb{R}\right), 0\right)$ and both functions $f$ on the same orbit of an action of the product on $s$ of these groups. Here, $\mathcal{G}\left(\mathbb{R}^{n_{s}}, 0\right)$ is the group of germs of diffeomorphisms of $\mathbb{R}^{n_{s}}$ at 0 and $\mathcal{G}\left(\left(\mathbb{R}^{n_{s}}, \mathbb{R}\right), 0\right)$ that of germs of functions $\mathbb{R}^{n_{s}} \rightarrow \mathbb{R}$ at 0 .

This action cannot be described explicitly. The adapted coordinates are the solution of an O.D.E in an infinite dimensional Banach space, see the proof of Theorem 3.7. This action is the natural action on the initial conditions of this O.D.E., which cannot be described simply on the solution of the O.D.E. themselves.

The holonomy algebra acts trivially on $\mathbf{X}_{m}$ if and only if $f$ does not depend on $x$.

Proof. The proof is the same as that of Corollary 5.15 p. 22, except for two points:

- Here, one does not prescribe that the coordinate-vectors $Y_{i}^{s}$, on $\mathcal{X}_{0}^{\perp}$, are the exponential of coordinate-vectors of $\mathrm{T}_{m} \mathcal{X}_{0}^{\perp}$, so the choice of the adapted coorinates depends on the choice, for each $s$, of the sections $\sigma_{0}^{s}$ of $\pi_{0}^{s}: \mathcal{Y}_{0}^{s} \rightarrow \check{\mathcal{Y}}_{0}^{s}$, on which $\mathcal{G}\left(\left(\mathbb{R}^{n_{s}}, \mathbb{R}\right), 0\right)$ acts simply transitively, and of the coordinates $\left(\check{y}_{i}^{s}\right)_{i=1}^{n_{s}}$ of $\check{\mathcal{Y}}_{\check{m}}^{s}$, on which $\mathcal{G}\left(\mathbb{R}^{n_{s}}, 0\right)$ acts simply transitively. So $\mathbb{R}^{n_{s}}$ is replaced by $\mathcal{G}\left(\left(\mathbb{R}^{n_{s}}, \mathbb{R}\right), 0\right)$ and $\mathrm{SO}\left(n_{s}, \mathbb{R}\right)$ by $\mathcal{G}\left(\mathbb{R}^{n_{s}}, 0\right)$.

- We will see in Proposition 7.4 that the choice of $\gamma$ amounts to that of (iii). Some comments about the meaning of $\gamma$ lead to that proposition, which completes the proof.

A simple means to obtain a reducible-indecomposable Lorentzian metric as described in (7.1) is to take $g=g^{0}+\sum_{s=1}^{k} g_{z}^{s}+2 \mathrm{~d} x \mathrm{~d} z$. Such a germ of metric is a Riemannian fibration over $\mathcal{B}=\left\{(x, 0, \ldots, 0, z) /(x, z) \in I^{2}\right\}, 2 \mathrm{~d} x \mathrm{~d} z$ being the (Lorentzian) metric of $\mathcal{B}$, and the (Riemannian) metric on each fibre depending only on the point in the fibre and the coordinate $z$ of $\mathcal{B}$. Not any Lorentzian reducible-indecomposable metric can be written in this form. The obstruction is exactly the bilinear form $\beta:\left(Y, Y^{\prime}\right) \mapsto g\left(R(Y, Z) Y^{\prime}, Z\right)$ involved in Corollary 7.2, (iii).

Now in adapted coordinates, this obstruction is also exactly given by $\gamma$, which conversely corresponds only to this obstruction. Let $g$ be a metric satisfying (7.1). Let us set $\overline{\mathbf{Y}}=$ $+_{r \neq s \text { or } r=s=0} \mathbf{Y}^{s} \otimes \mathbf{Y}^{r}$ and at some $p$, take $Y \otimes Y^{\prime} \in \overline{\mathbf{Y}}_{p}$ i.e. $Y \in \mathbf{Y}_{p}^{s}$ and $Y^{\prime} \in \mathbf{Y}_{p}^{r}$ with $r \neq s$ or $r=s=0$. By Lemma 4.1, $g\left(R(Y, Z) Y^{\prime}, Z\right)$ only depends on the class of $Z$ modulo $\mathbf{X}_{p}^{\perp}$. So, along each leaf $\mathcal{X}_{z}^{\perp}$ of $\mathcal{X}^{\perp}$, a bilinear symmetric form $\beta_{z}$ is defined, up to proportionality, by: $\beta_{z}\left(Y, Y^{\prime}\right)=g\left(R(Y, Z) Y^{\prime}, Z\right)$. Along $\mathcal{X}_{z}^{\perp}, \beta_{z}$ is a symmetric section of $\overline{\mathbf{Y}}^{*}=+_{r \neq s \text { or } r=s=0} \mathbf{Y}^{s *} \otimes \mathbf{Y}^{r *}$.

7.3 Remark By Lemma 4.3, there exists a closed 1-form $\alpha_{z}$ such that $\beta_{z}=\left(D \alpha_{z}\right) \mid \overline{\mathbf{Y}}$. This covariant derivative of $\alpha_{z}$ is independent of the choice of $g$; indeed, by (7.1) and by Lemma 4.1, the $\mathbf{Y}^{s}$ are parallel along the integral leaves of $\mathbf{Y}^{0}+\left({ }_{r \neq s} \mathbf{Y}^{r}\right)$, so if at some $p, Y \otimes Y^{\prime} \in \overline{\mathbf{Y}}$, a parallel extension of $Y$ (resp. $Y^{\prime}$ ) in the direction of $Y^{\prime}$ (resp. $Y$ ) is independent of $g$. With such extensions, $D_{Y} \alpha_{z}\left(Y^{\prime}\right)=L_{Y} \alpha\left(Y^{\prime}\right)$ and, if $f$ is a function, $L_{Y} L_{Y^{\prime}} f$ does not depend on the choice of $g$. With $f_{z}$ such that $\mathrm{d} f_{z}=\alpha_{z}$, in that sense, $\beta_{z}\left(Y, Y^{\prime}\right)=L_{Y} L_{Y^{\prime}} f_{z}=L_{Y^{\prime}} L_{Y} f_{z}$. 
7.4 Proposition By Lemma 4.3, the forms $\beta_{z}$ must be of the form $\beta_{z}\left(Y, Y^{\prime}\right)=L_{Y} L_{Y^{\prime}} f_{z}=$ $L_{Y^{\prime}} L_{Y} f_{z}$ with $f_{z}$ some function. This is the only constraint, i.e. $f_{z}$ may be any function. Recall that as $\left(Y, Y^{\prime}\right) \in \mathbf{Y}^{s} \times \mathbf{Y}^{r}$ with $r \neq s$ or $r=s=0$, the operator " $L_{Y} L_{Y^{\prime}}$ " is tensorial in $Y$ and $Y^{\prime}$, by Remark 7.3 above.

Proof. The family $\left(f_{z}\right)_{z \in I}$ being given, you must adjust, in adapted coordinates, the family of 1-forms $\left(\gamma_{z}\right)_{z \in I}$, so that $g\left(R(Z, Y) Y^{\prime}, Z\right)=L_{Y} L_{Y^{\prime}} f_{z}$ for $Y \otimes Y^{\prime} \in \overline{\mathbf{Y}}$. The wished family $\left(\gamma_{z}\right)_{z \in I}$ satisfies an O.D.E. of the type of (6.9), which has a solution by the same argument as that given at the end of the proof of Theorem 5.14 (b), after Lemma 6.2 p. 27.

7.5 Remarks (i) Each $f_{z}$, so the (local) failure of $g$ to be a Riemannian fibration as said above, is defined up to a function $\widetilde{f}_{z}=\sum_{i=1}^{k} \widetilde{f}_{z}^{s}$, where each $\widetilde{f}_{z}^{s}$ depends only on $y^{s}$.

(ii) This local failure is parametrized by $f_{z}$, or equivalently by $\beta_{z}$. In turn, $\gamma$ is given by $\beta_{z}$ through Relation (3.3). So $\gamma$ parametrizes also exactly this local failure.

(iii) As in Corollary 5.15, we could also take, in Corollary 7.2, the families $\left(\left(\check{g}_{z}^{s}\right)_{z \in I}\right)_{s=1}^{k}$ such that at $z=0, y^{s}$ are normal coordinates for $\check{g}_{0}^{s}$ and that $\left(\frac{\mathrm{d} \check{g}^{s}}{\mathrm{~d} t}\right)_{\mid t=0}(\vec{r}, \vec{r})=0$, with $\vec{r}$ the normal radius vector of $\mathbb{R}^{n_{s}}$. If so, the group $\mathcal{G}\left(\mathbb{R}^{n_{s}}, 0\right) \times \mathcal{G}\left(\left(\mathbb{R}^{n_{s}}, \mathbb{R}\right), 0\right)$ is replaced by $\mathrm{SO}_{n_{s}}(\mathbb{R}) \times \mathbb{R}^{n_{s}}$ in the following.

(iv) We could also give a version of Corollary 5.15 involving the family of functions $f_{z}$ instead of the family of forms $\gamma_{z}$. We let it to the reader.

\section{7. b A simpler formulation of Theorem 5.14 in a particular case}

Using Ebin's Slice Theorem, we can also make the terms $\operatorname{tr}_{\check{g}_{z}^{s}}\left(\mathrm{~d}^{\check{D}_{z}^{s}}\left(\frac{\mathrm{d}}{\mathrm{d} z} \check{g}_{z}^{s}\right)\left(\cdot, J_{z}^{s} \cdot, \check{Y}_{i}^{s}\right)\right)$ appearing in Definition 5.9 a bit more explicit. We recall the infinitesimal part of Ebin's Theorem.

7.6 Theorem (Ebin, [E68]) If $(\mathcal{M}, g)$ is a Riemannian manifold, $S^{2}\left(T^{*} \mathcal{M}\right)=\operatorname{Im} \delta^{*} \oplus$ $\operatorname{ker} \delta$, where $\delta$, called divergence, is the formal adjoint of $\delta^{*}$ defined in 6.3.

Therefore, if $g_{t}$ is a variable metric and $h_{t}=\frac{\mathrm{d} g_{t}}{\mathrm{~d} t}$, at each $t, h_{t}$ may be viewed as the sum of a deformation by a diffeomorphism (of the form $-2 \delta_{t}^{*} V_{t}$ by (6.11)) and of an "intrinsic deformation", in the sense "divergence-free deformation": there exists a vector field $V_{t}$ such that $h_{t}=-2 \delta_{t}^{*} V_{t}+\widetilde{h}_{t}$ with $\delta_{t} \widetilde{h}_{t}=0 ; \delta_{t}^{*}$ and $\delta_{t}$ being associated with $g_{t}$. If the $\left(J_{t}, g_{t}\right)$ are Kähler, applying this to $\operatorname{tr}_{g_{t}}\left(\mathrm{~d}^{D_{t}}\left(\frac{\mathrm{d}}{\mathrm{d} t} g_{t}\right)\left(\cdot, J_{t}, U\right)\right)$, we get:

7.7 Proposition We take $V_{t}$ such that $h_{t}=-2 \delta_{t}^{*} V_{t}+\widetilde{h}_{t}$ with $\delta_{t} \widetilde{h}_{t}=0$. Then:

$$
\begin{gathered}
\operatorname{tr}_{g_{t}} \mathrm{~d}^{D_{t}} \widetilde{h}_{t}\left(\cdot, J_{t} \cdot, U\right)=-L_{J_{t} U}\left(\operatorname{tr}_{g_{t}} \widetilde{h}_{t}\right), \\
\operatorname{tr}_{g_{t}}\left(\mathrm{~d}^{D_{t}} h_{t}\left(\cdot, J_{t} \cdot, U\right)\right)=L_{U} \operatorname{tr}_{g_{t}}\left(\mathrm{~d} V_{t}^{b_{t}}\left(\cdot, J_{t} \cdot\right)\right)-2 \operatorname{tr}\left(R_{t}\left(U, V_{t}\right) \circ J_{t}\right)-L_{J_{t} U}\left(\operatorname{tr}_{g_{t}} \widetilde{h}_{t}\right) .
\end{gathered}
$$

Relation (7.2) has two interests: the meaning of the right-hand side is clearer than that of the left-hand side and, in case we deal not only with germs of metrics, but with metrics on some given manifold, and if the quotient metric $\check{g}^{s}$ is a metric on some compact manifold, then $\operatorname{tr}_{\check{g}_{z}^{s}} \widetilde{\breve{h}}_{z}^{s}=0$. Indeed, $\widetilde{h}_{z}^{s}$ is an infinitesimal deformation of a (Ricci-flat, thus) Einstein compact manifold, according to Definition 12.29 of [Bes87], thus is trace free by Theorem 12.30 of the same book. So in this case, the term $\operatorname{tr}_{\check{g}_{z}^{s}}\left(\mathrm{~d}^{\check{D}_{z}^{s}}\left(\frac{\mathrm{d}}{\mathrm{d} z} \check{g}_{z}^{s}\right)\left(\cdot, J_{z}^{s} \cdot, \check{Y}_{i}^{s}\right)\right)$ depends only on the vector field $V_{z}$, i.e. on the projection of $\frac{\mathrm{d} \check{g}_{z}^{s}}{\mathrm{~d} z}$ on $\operatorname{Im} \delta^{*}$, not on the intrinsic deformation of $g_{z}$. 
Proof. (7.3) Follows from (7.2) and Lemma 6.4. Let us show (7.2) i.e., dropping the tilde on $h$ and the index $t: \delta h=0 \Rightarrow \operatorname{tr}_{g} \mathrm{~d}^{D} h(\cdot, J \cdot, U)=L_{J U}\left(\operatorname{tr}_{g} h\right)$. Let $h=h_{\mathrm{H}}+h_{\mathrm{S}}$ the decomposition of $h$ into its hermitian part: $h_{\mathrm{H}}(J \cdot, J \cdot)=h_{\mathrm{H}}(\cdot, \cdot)$ and skew-hermitian part: $h_{\mathrm{S}}(J \cdot, J \cdot)=-h_{\mathrm{S}}(\cdot, \cdot)$. Immediately, $\operatorname{tr}_{g} h_{\mathrm{S}}=0$; besides, as $\delta h=0$ and by Lemma 12.94 of [Bes87], $\delta h_{\mathrm{H}}=\delta h_{\mathrm{S}}=0$. Let $E$ be the space on which $g$ is defined; $\operatorname{dim} E=2 d$. By definition of $\delta$, and recalling that $\delta h(U)=-\operatorname{tr}_{g} \delta^{*} h(\cdot, \cdot, U)$ :

$$
\left(X_{i}\right)_{i=1}^{2 d} \text { is } g \text {-orthonormal } \Rightarrow \delta h(U)=-\left(L_{U}\left(\operatorname{tr}_{g} h\right)+2 \sum_{i=1}^{2 d}\left(D_{X_{i}} h\right)\left(X_{i}, U\right)\right)
$$

Let $\left(Y_{i}\right)_{i=1}^{d}$ be an orthonormal basis of $(E, J)$ as a hermitian space. $\operatorname{As}_{\operatorname{tr}_{g}} \mathrm{~d}^{D} h(\cdot, \cdot, U)$ is $\mathbb{R}$-bilinear, skew-symmetric, it comes that $\operatorname{tr}_{g} \mathrm{~d}^{D} h(\cdot, J \cdot, U)=2 \sum_{i=1}^{d} \mathrm{~d}^{D} h\left(Y_{i}, J Y_{i}, U\right)$. Now:

$$
\begin{aligned}
& \mathrm{d}^{D} h\left(Y_{i}, J Y_{i}, U\right) \\
= & D_{Y_{i}} h_{\mathrm{H}}\left(J Y_{i}, U\right)-D_{J Y_{i}} h_{\mathrm{H}}\left(Y_{i}, U\right)+D_{Y_{i}} h_{\mathrm{S}}\left(J Y_{i}, U\right)-D_{J Y_{i}} h_{\mathrm{S}}\left(Y_{i}, U\right) \\
= & -\left(D_{Y_{i}} h_{\mathrm{H}}\left(Y_{i}, J U\right)+D_{J Y_{i}} h_{\mathrm{H}}\left(J Y_{i}, J U\right)\right)+\left(D_{Y_{i}} h_{\mathrm{S}}\left(Y_{i}, J U\right)+D_{J Y_{i}} h_{\mathrm{S}}\left(J Y_{i}, J U\right)\right)
\end{aligned}
$$

So, by (7.4) and as $\left(\left(Y_{i}\right)_{i=1}^{d},\left(J Y_{i}\right)_{i=1}^{d}\right)$ is $g$-orthonormal, $\operatorname{tr}_{g} \mathrm{~d}^{D} h(\cdot, J \cdot, U)=\left(L_{J U} \operatorname{tr}_{g} h_{\mathrm{H}}+\right.$ $\left.\delta h_{\mathrm{H}}(J U)\right)-\left(L_{J U} \operatorname{tr}_{g} h_{\mathrm{S}}+\delta h_{\mathrm{S}}(J U)\right)$. As $\delta h_{\mathrm{H}}=\delta h_{\mathrm{S}}=0$ and $\operatorname{tr}_{g} h_{\mathrm{S}}=0$, we are done.

\section{7. c A look on some low-dimensional cases; explicit examples}

If $g$ is a metric with holonomy representation of type 3 or 4 and if for some $s \in \llbracket 1, k^{\prime} \rrbracket$, $\operatorname{dim} \check{\mathbf{Y}}^{s}=2$, then for any $z, \check{g}_{z}^{s}$ is Ricci-flat hence flat so $\check{g}_{z}^{s}=\left(\varphi_{z}^{-1}\right)^{*} \check{g}_{0}^{s}$ with $\check{g}_{s}^{0}$ the flat metric of $\mathbb{R}^{2}$, written for example in the canonical coordinates. As $R_{z}$ vanishes, and by (6.2) and (6.5): $\operatorname{tr}_{\check{g}_{z}^{s}}\left(\mathrm{~d}^{\check{D}_{z}^{s}}\left(\frac{\mathrm{d}}{\mathrm{d} z} \check{g}_{z}^{s}\right)\left(\cdot, J_{z}^{s} \cdot, U\right)\right)=L_{\varphi_{z}^{-1}(U)} \Delta_{\check{g}_{0}^{s}} \Im f_{z}$, with $f_{z} \in C^{\infty}\left(\mathbb{R}^{2}, \mathbb{C}\right)$ such that $\frac{\mathrm{d} \varphi_{z}}{\mathrm{~d} z}=\left(\partial f_{z}+\overline{\partial f_{z}}\right)^{b}=\operatorname{grad}_{\check{g}_{0}^{s}} \Re f_{z}+\operatorname{grad}_{\breve{\omega}_{0}^{s}} \Im f_{z}$, where $\operatorname{grad}_{\breve{\omega}_{0}^{s}}$ is the symplectic gradient with respect to the canonical symplectic form $\check{\omega}_{0}^{s}=\check{g}_{0}^{s}\left(\cdot, J_{0}^{s} \cdot\right)$. One may also notice that if $V_{z}$ is the vector field of which $\left(\varphi_{z}\right)_{z \in I}$ is the flow, $\operatorname{tr}_{\check{g}_{z}^{s}}\left(\mathrm{~d} V_{z}^{b_{z}^{s}}\left(\cdot, J_{z}^{s} \cdot\right)\right)=\operatorname{rot}_{\check{g}_{z}^{s}} V_{z}$ by definition of the rotational.

We may then end, by this means, with an explicit family of examples of reducible-indecomposable Lorentzian metrics on $\mathbb{R}^{5}$, with holonomy representation of "exceptional" type 4 . In adapted coordonates $\left(x, y_{0}, y_{1}^{1}, y_{1}^{2}, z\right)$, here simply denoted by $\left(x, y_{0}, y_{1}, y_{2}, z\right)$, the metric $g$ reads:

$$
\operatorname{Mat}(g)=\left(\begin{array}{c|c|cc|c}
0 & 0 & 0 & 0 & 1 \\
\hline 0 & 1 & 0 & 0 & \gamma_{0} \\
\hline 0 & 0 & \check{g}_{1,1} & \check{g}_{1,2} & \gamma_{1} \\
0 & 0 & \check{g}_{2,1} & \check{g}_{2,2} & \gamma_{2} \\
\hline 1 & \gamma_{0} & \gamma_{1} & \gamma_{2} & 0
\end{array}\right) ; \text { besides we denote } \check{G}:=\left(\begin{array}{cc}
\check{g}_{1,1} & \check{g}_{1,2} \\
\check{g}_{2,1} & \check{g}_{2,2}
\end{array}\right) \text {, }
$$

with, at $z=0, \gamma_{0}=\gamma_{1}=\gamma_{2} \equiv 0$ and $\check{G} \equiv I_{2}$ and, on $\left\{x=y_{0}=0\right\}, \gamma_{1}=\gamma_{2} \equiv 0$. (Caution: to simplify the notation, we turned it into: $\gamma_{0}=1, \gamma_{0}^{1} \rightsquigarrow \gamma_{0}, \gamma_{1}^{1} \rightsquigarrow \gamma_{1}, \gamma_{1}^{2} \rightsquigarrow \gamma_{2}$.) The only - arbitrary - datum to provide is that of the one-parameter family $\left(W_{z}\right)_{z \in \mathbb{R}}$ of vector fields on $\mathbb{R}^{2}=\operatorname{span}\left(Y_{1}, Y_{2}\right)$, derivative in $z$ of the one-parameter family of diffeomorphisms $\left(\varphi_{z}\right)_{z \in \mathbb{R}}$. Indeed, according to all that precedes - Theorems 3.7 and 5.14, Relation (6.11) of Lemma 6.4, Proposition 7.7 and the remarks opening this paragraph- $\mathfrak{h}(g)$ is of type 4, with factor $\lambda \in \mathbb{R}^{*}$ if and only if 
(i) if $p \in\{z=0\}=\mathcal{X}_{0}^{\perp}$, for any $z$, for $i \in\{1,2\},\left(L_{Y_{i}} L_{Z} \gamma_{0}\right)_{\mid \varphi_{z}(p)}=\lambda\left(L_{Y_{i}} \operatorname{rot} W_{z}\right)_{\mid p} \not \equiv 0$

(ii) if $p \in\{z=0\}=\mathcal{X}_{0}^{\perp}$, for any $z,\left(\frac{\mathrm{d}}{\mathrm{d} z} \check{G}\right)_{\mid \varphi_{z}(p)}=-2 \delta^{*}\left(W_{z}^{b}\right)_{\mid p}$,

(iii) On $\{z=0\}$, for all $i, \gamma_{i} \equiv 0$ and $\breve{G} \equiv I_{2}$; moreover $\gamma_{0} \equiv 0$ on the subspace $\left\{y_{1}=y_{2}=0\right\}$ and $\left\{\gamma_{1} \equiv \gamma_{2} \equiv 0\right\}$ on the subspace $\left\{y_{0}=0\right\}$. Besides, for all $i \in\{1,2\}$, the value on $\mathbb{R}^{5}$ of the $\gamma_{i}$ is given by: $\partial \gamma_{i} / \partial y_{0}=\partial \gamma_{0} / \partial y_{i}$.

Let us now take for example a $\left(W_{z}\right)_{z \in \mathbb{R}}$ of the form $W_{z}=\left(a\left(y_{2}\right) b(z), 0\right)$. Then if $p \in \operatorname{span}\left(Y_{1}, Y_{2}\right)$ has coordinates $\left(y_{1}, y_{2}\right), \varphi_{z}(p)=\varphi_{z}\left(y_{1}, y_{2}\right)=\left(y_{1}+a\left(y_{2}\right) B(z), y_{2}\right)$, where $B(z)=\int_{0}^{z} b(t) \mathrm{d} t$, as $\frac{\mathrm{d} \varphi_{z}}{\mathrm{~d} z} \mid q=\left(W_{z}\right)_{\mid q}$. Notice also that, as $\gamma_{0} \equiv 0$ on $\gamma_{1} \equiv \gamma_{2} \equiv 0$, (i) becomes: $\left(L_{Z} \gamma_{0}\right)_{\mid \varphi_{z}(p)}=\lambda\left(\operatorname{rot} W_{z}\right)_{\mid p} \not \equiv 0$ and $\operatorname{rot} W_{z} \not \equiv$ const. Let us also recall that, in our flat, 2-dimensional framework:

- $\operatorname{rot} W_{z}=\frac{\partial w_{1}}{\partial y_{2}}-\frac{\partial w_{2}}{\partial y_{1}}$,

- $\delta^{*} W_{z}^{b}=\frac{1}{2}\left(\begin{array}{cc}2 \frac{\partial w_{1}}{\partial y_{1}} & \frac{\partial w_{1}}{\partial y_{2}}+\frac{\partial w_{2}}{\partial y_{1}} \\ \frac{\partial w_{1}}{\partial y_{2}}+\frac{\partial w_{2}}{\partial y_{1}} & 2 \frac{\partial w_{2}}{\partial y_{2}}\end{array}\right)$.

Simple computations show then that (i), (ii) and (iii) above are respectively equivalent to:

(i) $\gamma_{0}=\lambda a^{\prime}\left(y_{2}\right) B(z)$ and $a^{\prime \prime}\left(y_{2}\right) \not \equiv 0 \quad$ (notice that, by definition, $B(0)=0$ ),

(ii) $\check{G}_{z}=\left(\begin{array}{cc}1 & a^{\prime}\left(y_{2}\right) B(z) \\ a^{\prime}\left(y_{2}\right) B(z) & 1\end{array}\right)$,

(iii) $\gamma_{1} \equiv 0$ and $\gamma_{2}=\lambda y_{0} a^{\prime \prime}\left(y_{2}\right) B(z)$.

7.8 Proposition Consequently, let $g$ be a Lorentzian metric on $\mathbb{R}^{5}$ given as above in adapted coordinates, and such that its characteristic vector field $\left(W_{z}\right)_{z \in \mathbb{R}}$ is of the form $\left(a\left(y_{2}\right) b(z), 0\right)$. Its holonomy algebra is of type 4 , with characteristic coefficient $\lambda \in \mathbb{R}^{*}$, if and only if the three points above are satisfied.

Similarly, let $g$ be the metric on $\mathbb{R}^{4}$ given (in adapted coordinates $\left(x, y_{1}, y_{2}, z\right)$ ) by:

$$
\operatorname{Mat}(g)=\left(\begin{array}{c|cc|c}
0 & 0 & 0 & \gamma_{0} \\
\hline 0 & \check{g}_{1,1} & \check{g}_{1,2} & \gamma_{1} \\
0 & \check{g}_{1,2} & \check{g}_{2,2} & \gamma_{2} \\
\hline \gamma_{0} & \gamma_{1} & \gamma_{2} & 0
\end{array}\right) \text {, with }\left\{\begin{array}{l}
\text { at } z=0, \gamma_{0} \equiv 1, \gamma_{1}=\gamma_{2} \equiv 0 \\
\text { and } \breve{G} \equiv I_{2} \text { (same notation as above); } \\
\text { at } x=0, \gamma_{1}=\gamma_{2} \equiv 0 .
\end{array}\right.
$$

We suppose that, as above, $\check{G}$ is the identity $I_{2}$ pushed by a family diffeomorphism $\left(\varphi_{z}\right)_{z \in \mathbb{R}}$, the derivative $\left(W_{z}\right)_{z \in \mathbb{R}}$ of which is of the form $\left(a\left(y_{2}\right) b(z), 0\right)$. One checks that:

7.9 Proposition The holonomy algebra of $g$ is of type 3, with coefficient $\lambda \in \mathbb{R}^{*}$, if and only if:

- $\gamma_{0}=u\left(y_{2}, z\right)$, with $u$ the unique solution of $u \frac{\partial^{2} \ln u}{\partial z \partial y_{2}}=\lambda a^{\prime}\left(y_{2}\right) b(z)$ equal to 1 on $\{z=0\} \cup\left\{y_{2}=0\right\}$ (see the end of the proof of Theorem 5.14 (b) p. 27), and with $a^{\prime \prime}\left(y_{2}\right) \not \equiv 0$,

- $\breve{G}$ is as in (ii) above,

- $\gamma_{1} \equiv 0$ and $\gamma_{2}=x \frac{\partial u}{\partial y_{2}}$. 


\section{References}

[BBI93] L. Bérard Bergery, A. Ikemakhen, On the Holonomy of Lorentzian Manifolds. Proc. of Symposia in Pure Mathematics 54, Part 2:27-39, 1993.

[Be55] M. Berger, Sur les groupes d'holonomie homogène des variétés à connexion affine et des variétés riemanniennes. (French) Bull. Soc. Math. France 83 (1955), 279-330.

[Be57] M. Berger. Les espaces symétriques non compacts. Ann. Sci. Ecol. Norm. Sup., 74:85-177, 1957.

[Bes87] A. L. Besse. Einstein Manifolds. Springer Verlag — Berlin, Heidelberg, 1987.

[Br96] R. Bryant, Classical, exceptional, and exotic holonomies: A status report. Besse, A. L. (ed.), Actes de la table ronde de géométrie différentielle en l'honneur de Marcel Berger, Luminy, France, 12-18 juillet 1992. Soc. Math. France. Sémin. Congr. 1, 93-165 (1996).

[Bo00] Ch. Boubel, Sur l'holonomie des variétés pseudo-riemanniennes, Thèse de doctorat, Université Nancy I, France, May 2000.

[BZ03] Ch. Boubel, A. ZEGHIB, Isometric actions of Lie subgroups of the Moebius group. Nonlinearity 17 (2004) 1677-1688.

[CP80] M. Cahen, M. Parker, Pseudo-Riemannian symmetric spaces. Memoirs of the American Mathematical Society, 24 (229), 1980.

[C24] E. CARTAN, Sur les variétés à connexion affine et la théorie de la relativité généralisée I et II, Ann. Sci. Ecol. Norm. Sup. 40, 325-412 (1923) and 41, 1-25 (1924).

[C26] E. Cartan, Les groupes d'holonomie des espaces généralisés, Acta. Math. 48, 1-42 (1926).

[E68] D. G. EbIn. The manifold of Riemannian metrics. 1970 Global Analysis, Proc. of Symposia in Pure Mathematics 15:11-40, 1968.

[G05] A. Galaev. Metrics that realize all types of Lorentzian holonomy, preprint, http://arxiv.org/abs/math.DG/0502575.

[HO56] J. Hano, H. OzeKI, On the holonomy group of linear connections, Nagoya Math. J. 10, 97-100, 1956.

[196] A. Ikemakhen, Examples of indecomposable non-irreducible Lorentzian manifolds. Ann. Sci. Math. Qué. 20, No.1, 53-66 (1996).

[LB70] A. Lascoux, M. Berger, Variétés kählériennes compactes. (French) Lecture Notes in Mathematics. 154. Berlin-Heidelberg-New York: Springer-Verlag, 1970.

[L03a] T. Leistner, Towards a classification of Lorentzian holonomy groups. Preprint, 2003, http://arxiv.org/ps/math.DG/0305139 and Part II: Semisimple, non-simple weak-Berger algebras. Preprint, 2003, http://arxiv.org/ps/math.DG/0309274.

[L03b] T. Leistner, Holonomy and Parallel Spinors in Lorentzian Geometry, thesis, HumboldtUniversität zu Berlin, 2003. Published (book) by Logos-Verlag, Berlin, 2004.

[dR52] G. DE Rham, Sur la réductibilité d'un espace de Riemann, Comm. Math. Helv. 26, 328-344 (1952).

[DO01] A. J. Di Scala, C. Olmos, The geometry of homogeneous submanifolds of hyperbolic space. Math. Z. 237, No.1, 199-209 (2001).

[S01a] L. SchwachнӧғER, Irreducible holonomy representations. Proceedings of the 20th Winter School "Geometry and Physics" (Srní, 2000). Rend. Circ. Mat. Palermo (2) Suppl. No. 66 (2001), 59-81

[S01b] L. SCHWACHHÖFER Connections with irreducible holonomy representations. Adv. Math. 160 (2001), no.1, 1-80. 
[W49] A. G. Walker. On parallel fields of partially null vector spaces. Q. J. Math., Oxf. II. Ser. 1, 69-79 (sept. 1949).

[W50] A. G. WALKer. Canonical form for a riemannian space with a parallel field of null planes, I and II. Q. J. Math., Oxf. II. Ser. 1, 69-79 and 147-152, 1950.

[W67] H. Wu. Holonomy groups of indefinite metrics. Pacific J. Math. 20:351-392, 1967.

[Z02] A. ZEGHib, Remarks on Lorentz symmetric spaces, Compos. Math. 140 (2004), no. 6, 16751678 